\title{
Two-loop RGEs with Dirac gaugino masses
}

\author{
Mark D. Goodsell \\ Theory Division, CERN, \\ CH-1211 Geneva 23, Switzerland \\ E-mail: mark.goodsell@cern.ch
}

ABSTRACT: The set of renormalisation group equations to two-loop order for general supersymmetric theories broken by soft and supersoft operators is completed. As an example, the explicit expressions for the RGEs in a Dirac gaugino extension of the (N)MSSM are presented.

Keywords: Supersymmetric gauge theory, Renormalization Group

ArXiv EPRINT: 1206.6697 


\section{Contents}

1 Introduction 1

2 Tadpole RGEs $\quad 4$

2.1 Tadpole in non-supersymmetric theories 4

2.2 Translating from $\overline{\mathrm{MS}}$ to $\overline{\mathrm{DR}}^{\prime} \quad 5$

2.3 Result 6

3 RGEs of a Dirac gaugino extension of the (N)MSSM $\quad 8$

3.1 Dirac gaugino masses $\quad 9$

$\begin{array}{ll}3.2 \text { Tadpole equation } & 10\end{array}$

$\begin{array}{lll}4 \text { Conclusions } & 10\end{array}$

$\begin{array}{ll}\text { A General non-supersoft operators } & 11\end{array}$

B Two-loop RGEs for SUSY and standard soft terms 12

B.1 Anomalous dimensions 12

$\begin{array}{ll}\text { B.2 Gauge couplings } & 14\end{array}$

$\begin{array}{ll}\text { B.3 Gaugino mass parameters } & 14\end{array}$

$\begin{array}{ll}\text { B.4 Trilinear superpotential parameters } & 15\end{array}$

$\begin{array}{ll}\text { B.5 Bilinear superpotential parameters } & 16\end{array}$

$\begin{array}{ll}\text { B.6 Linear superpotential parameters } & 17\end{array}$

$\begin{array}{ll}\text { B.7 Trilinear soft-breaking parameters } & 17\end{array}$

$\begin{array}{ll}\text { B.8 Bilinear soft-breaking parameters } & 22\end{array}$

B.9 Linear soft-breaking parameters 23

$\begin{array}{ll}\text { B.10 Soft-breaking scalar masses } & 25\end{array}$

\section{Introduction}

Models with Dirac gaugino masses are attractive for a number of reasons. From a topdown model-building perspective, this is because they preserve R-symmetry, and so allow for simple supersymmetry-breaking sectors. This has attracted much interest in the literature [1-31]. On the other hand, if gauginos are found at the LHC, it must be determined whether they are of Majorana or Dirac type [27, 32-35]. Moreover, with the current results from the LHC, Dirac mass terms allow the preservation of naturalness by having lower bounds on the gluino and squark masses in the Majorana case (they are "super-safe" [29]); by increasing the Higgs mass due to additional couplings [4, 5, 8, 21]; and because they do not cause the Higgs masses to run as strongly [4, 16, 36]. 
Particularly out of a desire to study the naturalness and Higgs sectors of such theories, it is important to know the renormalisation group equations (RGEs) for them. The purpose of this paper is to complete the set of RGEs to two-loop order.

The standard soft supersymmetry-breaking mass terms are well known:

$$
\mathcal{L}_{\text {standard }}=-\frac{1}{2}\left(m^{2}\right)_{j}^{i} \phi_{i} \phi^{j}-\frac{1}{2} M \lambda_{A} \lambda_{A}-\frac{1}{2} B^{i j} \phi_{i} \phi_{j}-\frac{1}{6} A^{i j k} \phi_{i} \phi_{j} \phi_{k}+\text { h.c. }
$$

where the $\phi_{i}$ are the scalars of chiral multiplets $\Phi_{i}=\phi_{i}+\sqrt{2}\left(\theta \psi_{i}\right)+\ldots$ and $\phi^{i} \equiv\left(\phi_{i}\right)^{*} ; \lambda_{A}$ are gauginos. The above includes Majorana gaugino masses $M$. However, Dirac gaugino masses fall under the category of "non-standard" soft terms:

$$
\mathcal{L}_{\text {non-standard }}=-t^{i} \phi_{i}-m_{D}^{i A} \psi_{i} \lambda_{A}-\frac{1}{2} r^{i j}{ }_{k} \phi_{i} \phi_{j} \phi^{k}+\text { h.c. }
$$

General choices of these terms will lead to quadratic divergences in singlet tadpoles, and this has often led to the terms being neglected or only included in theories without singlets. However, to give a Dirac mass to the Bino, a singlet superfield must be included, so it is necessary to worry about this issue. On the other hand, when supersymmetry is spontaneously broken, the expectation is that no quadratic divergences should be generated, and indeed it is generically found that only supersoft [4] operators are generated:

$$
\begin{aligned}
\mathcal{L}_{\text {supersoft }} & \supset \int \mathrm{d}^{2} \theta \sqrt{2} m_{D}^{i A} \theta^{\alpha} \Phi_{i} W_{A \alpha}+\text { h.c. } \\
& \supset-m_{D}^{i A} \psi_{i} \lambda_{A}+\sqrt{2} m_{D}^{i A} \phi_{i} D_{A}+\text { h.c. }
\end{aligned}
$$

where $D_{A}$ is the D-term of the gauge group to which the adjoint couples with adjoint index ' $A$ '. They lead to a particular structure of non-standard soft terms such that the quadratic divergences exactly cancel. These interestingly augment supersymmetric trilinear couplings; including a superpotential

$$
W=L^{i} \Phi_{i}+\frac{1}{2} \mu^{i j} \Phi_{i} \Phi_{j}+\frac{1}{6} Y^{i j k} \Phi_{i} \Phi_{j} \Phi_{k}
$$

the non-standard trilinear couplings are

$$
\begin{aligned}
& r_{k}^{i j}=Y^{i j m} \mu_{m k}+\sqrt{2} g\left(m_{D}^{i A}\left(t^{A}\right)_{k}^{j}+m_{D}^{j A}\left(t^{A}\right)_{k}^{i}\right) \\
& r_{i j}{ }^{k}=Y_{i j m} \mu^{m k}+\sqrt{2} g\left(m_{D i A}\left(t^{A}\right)_{j}^{k}+m_{D j A}\left(t^{A}\right)_{i}^{k}\right)
\end{aligned}
$$

where $\left(t^{A}\right)_{j}^{i}$ are the generators of the gauge group. Of course, the supersymmetric terms do not generate quadratic divergences - these are cancelled by fermion loops - and only receive wavefunction renormalisation. The would-be quadratic divergences from the nonsupersymmetric, supersoft, piece are not cancelled by fermion loops but instead vanish when they are all summed.

If there is a Fayet-Iliopoulos (FI) term $\xi_{Y}$ (where $Y$ denotes a $\mathrm{U}(1)$ index) then a contribution to the tadpole is generated of

$$
\Delta t^{a}=\sqrt{2} m_{D}^{a Y} \xi_{Y}
$$


Typically, however, any FI term generated can be absorbed into the soft masses; this equation shows that in the presence of Dirac gaugino mass terms it should also be absorbed into a shift of the tadpole. Interestingly, there is also a supersymmetric term that emulates a tadpole equal to $\mathcal{L} \supset-\mu^{i j} L_{j} \phi_{i}+$ c.c.; of course this only has wavefunction renormalisation, just as for the trilinear terms above.

An important point is that the supersoft operator also generates contributions to the standard soft breaking terms via the self-coupling: from integrating out the auxiliary $D$ field there are terms

$$
\mathcal{L} \supset-\left(m_{D}^{i A} \phi_{i}+m_{D i A} \phi^{i}\right)\left(m_{D}^{j A} \phi_{j}+m_{D j A} \phi^{j}\right)
$$

and so

$$
\begin{aligned}
\Delta\left(m^{2}\right)_{j}^{i} & =2 m_{D}^{i A} m_{D j A} \equiv 2\left(m_{D}^{2}\right)_{j}^{i} \\
\Delta B^{i j} & =2 m_{D}^{i A} m_{D}^{j A} \equiv 2\left(m_{D}^{2}\right)^{i j} .
\end{aligned}
$$

The two-loop renormalisation group equations (RGEs) for standard SUSY-breaking terms were derived some time ago [37-40], and later the generic RGEs for non-standard soft terms were calculated in the absence of singlets [41,42]. They also found that restricting the non-standard terms to only be generated from supersoft operators defined a renormalisation group invariant trajectory, explained by the holomorphic nature of the supersoft operator. This means that equations (1.5) and (1.8) are true at any renormalisation scale. The supersoft operator only obtains wavefunction renormalisation, so its beta-function is

$$
\beta_{m_{D}^{i A}}=\gamma_{j}^{i} m_{D}^{j A}+\frac{\beta_{g}}{g} m_{D}^{i A}
$$

where $\gamma_{j}^{i}$ is the anomalous dimension of the adjoint superfield, and $\beta_{g}$ is the beta-function for the gauge coupling. Thus in a theory with Dirac gaugino mass terms, the RGEs for the standard soft terms can be found and evolved ignoring the Dirac gaugino mass, and then at the scale of interest the shifts (1.8) can be applied to find the physical masses. In a theory without gauge singlets, this is then enough to determine all of the RGEs in the theory. However, when there are singlets - such as when there is a Dirac mass for the Bino - the RGE for the tadpole is also required, which is a non-standard term so may depend upon the Dirac gaugino mass, and not just via the above shifts. Considering that the singlet superfield may couple to the Higgs via a term

$$
W \supset \lambda_{S} \mathbf{S H}_{\mathbf{u}} \cdot \mathbf{H}_{\mathbf{d}}
$$

it is clear that knowing the size of the singlet tapdole (indeed, ensuring that it is not too large, since it is not protected by R symmetry for example) is vital in order to investigate electroweak symmetry breaking and determine the Higgs mass. The main result of this paper is to determine these RGEs to two-loop order.

In section 2 the result is presented, with an explanation of how the different terms arise. The method used is that of Martin and Vaughn [38], deriving the RGE for a tadpole 
in a general renormalisable theory from the expressions given in [43-46], then specialising to the softly broken supersymmetric case, transforming from $\overline{\mathrm{MS}}$ to $\overline{\mathrm{DR}}^{\prime}$. To do this the rules given in [47] will be used, and augmented with a new rule for Dirac gaugino mass terms. In section 3 and appendix B the RGEs are derived for a minimal Dirac gaugino extension of the supersymmetric standard model with rather general couplings. In addition, appendix A contains some discussion of the effect when the non-standard terms are not of the supersoft type.

\section{Tadpole RGEs}

There are several ways to derive the RGEs for softly broken supersymmetric models: by diagrams in component fields; by supergraphs; by RG invariance of the effective potential [41]; or by translating the results from a general renormalisable theory into the broken supersymmetric case. This last approach is the one adopted here, although the one-loop result was checked via the effective potential method.

\subsection{Tadpole in non-supersymmetric theories}

The first step in calculating the tadpole is to write down the expression in general nonsupersymmetric theories. This can be derived using spurions from the RGEs for the quartic coupling in a general renormalisable theory given in [43-46]. Such a theory with real scalars $\phi_{a}$ and complex fermions $\psi_{i}$ has couplings

$$
\begin{aligned}
\mathcal{L} \supset & -\frac{1}{24} \lambda_{a b c d} \phi_{a} \phi_{b} \phi_{c} \phi_{d}-\frac{1}{6} h_{a b c} \phi_{a} \phi_{b} \phi_{c}-\frac{1}{2} m_{a b}^{2} \phi_{a} \phi_{b}-t_{a} \phi_{a} \\
& -\frac{1}{2}\left[\left(m_{f}\right)_{i j}\left(\psi_{i} \psi_{j}\right)+\left(Y^{a}\right)_{i j} \phi_{a}\left(\psi_{i} \psi_{j}\right)+h . c .\right]
\end{aligned}
$$

in addition to a gauge coupling $g$.

The one-loop tadpole RGE is found to be

$$
(4 \pi)^{2} \beta_{t_{a}}=2 \kappa Y_{2}(S)^{a b} t_{b}+h_{a e f} m_{e f}^{2}-4 \kappa \operatorname{Tr}\left(Y_{a} m_{f}^{\dagger} m_{f} m_{f}^{\dagger}\right)-4 \kappa \operatorname{Tr}\left(Y_{a}^{\dagger} m_{f} m_{f}^{\dagger} m_{f}\right)
$$

where $\kappa=1 / 2$ for Weyl fermions (or 1 for Dirac fermions) and

$$
Y_{2}(S)^{a b} \equiv \frac{1}{2} \operatorname{Tr}\left(Y^{\dagger a} Y^{b}+Y^{\dagger b} Y^{a}\right)
$$

The two-loop tadpole RGE is

$$
\begin{aligned}
\beta_{t_{a}}= & \left(\gamma_{S}^{(2)}\right)_{a}^{b} t_{b} \\
& +h_{a e f} m_{e g}^{2}\left(8 g^{2} C_{2}^{f g}-4 \kappa Y_{2}(S)^{f g}\right)-\frac{g^{2}}{2} h_{a e f} h_{e g h} h_{f g h}-g^{2} m_{e f}^{2} \lambda_{a e g h} h_{f g h} \\
& +4 \kappa\left(2 \bar{H}_{a}^{\lambda}+H_{a}^{Y}+2 \bar{H}_{a}^{Y}+2 H_{a}^{3}-g^{2} H_{a}^{F}\right)
\end{aligned}
$$


where now $C_{2}{ }^{f g}$ is the quadratic casimir of the gauge group for the representation carried by fields $f$ and $g$, and

$$
\begin{aligned}
\left(\gamma_{S}^{(2)}\right)_{a}^{b} & \equiv \frac{1}{24} \lambda_{a c d e} \lambda_{b c d e}-\frac{3}{2} \kappa \operatorname{Tr}\left(Y^{a} Y^{\dagger b} Y^{c} Y^{\dagger c}\right)-\kappa \operatorname{Tr}\left(Y^{a} Y^{\dagger c} Y^{b} Y^{\dagger c}\right)+5 \kappa g^{2} \operatorname{Tr}\left(C_{2} Y^{a} Y^{\dagger b}\right)+c . c . \\
\bar{H}_{a}^{\lambda} & =2 h_{a e f} \operatorname{Tr}\left(m_{f} Y^{\dagger e} m_{f} Y^{\dagger f}\right)+4 m_{e f}^{2} \operatorname{Tr}\left(Y^{a} Y^{\dagger e} m_{f} Y^{\dagger f}\right)+c . c . \\
H_{a}^{Y} & =\operatorname{Tr}\left(Y_{2}(F) m_{f}^{\dagger} Y^{a} m_{f}^{\dagger} m_{f}+Y_{2}(F) m_{f}^{\dagger} m_{f} m_{f}^{\dagger} Y^{a}\right)+c . c . \\
\bar{H}_{a}^{Y} & =\frac{1}{2} \operatorname{Tr}\left(Y^{\dagger e} Y^{a} Y^{\dagger e} m_{f} m_{f}^{\dagger} m_{f}+2 Y^{\dagger e} m_{f} Y^{\dagger e} Y^{a} m_{f}^{\dagger} m_{f}+Y^{\dagger e} m_{f} Y^{\dagger e} m_{f} Y^{\dagger a} m_{f}\right)+c . c . \\
H_{a}^{3} & =\operatorname{Tr}\left(Y^{a} m_{f}^{\dagger} Y^{e} m_{f}^{\dagger} m_{f} Y^{\dagger e}\right)+c . c . \\
H_{a}^{F} & =4 \operatorname{Tr}\left(C_{2} Y^{a} m_{f}^{\dagger} m_{f} m_{f}^{\dagger}\right)+c . c .
\end{aligned}
$$

Here

$$
Y_{2}(F)_{i j} \equiv\left(Y^{\dagger a} Y^{a}\right)_{i j}
$$

\subsection{Translating from $\overline{\mathrm{MS}}$ to $\overline{\mathrm{DR}}^{\prime}$}

To specialise the above expressions to the supersymmetric case, they must be transformed to a complex basis (by summing repeated indices over both raised and lowered indices alternately) and insert the SUSY couplings. These can be written as block diagonal matrices, with the top row/left column corresponding to gauge indices, and bottom row/right column matter indices. The Yukawa matrices become

$$
\begin{aligned}
Y_{i} & =\sqrt{2} g\left(\begin{array}{cc}
0 & \left(t^{A}\right)_{i}^{j} \\
\left(t^{A}\right)_{i}^{j} & 0
\end{array}\right) & Y^{\dagger i} & =\sqrt{2} g\left(\begin{array}{cc}
0 & \left(t^{A}\right)_{j}^{i} \\
\left(t^{A}\right)_{j}^{i} & 0
\end{array}\right) \\
Y^{i} & =\left(\begin{array}{cc}
0 & 0 \\
0 & Y^{i j k}
\end{array}\right) & Y_{i}^{\dagger} & =\left(\begin{array}{cc}
0 & 0 \\
0 & Y_{i j k}
\end{array}\right)
\end{aligned}
$$

where $\left(t^{A}\right)_{i}^{j}$ are the gauge generators. With the definitions

$$
\begin{aligned}
\left(Y_{2}\right)_{b}^{a} & \equiv Y^{a c d} Y_{b c d} \\
S_{2} \delta^{A B} & \equiv\left(t^{A}\right)_{i}^{j}\left(t^{B}\right)_{j}^{i}
\end{aligned}
$$

then

$$
\begin{aligned}
Y_{2}(S)_{b}^{a} & \rightarrow\left(Y_{2}\right)_{b}^{a}+4 g^{2} C_{2 g}^{f} \\
Y_{2}(F) & \rightarrow\left(\begin{array}{cc}
2 g^{2} S_{2} & \mathbf{1} \\
9 & 2 g^{2} C_{2}+Y_{2}
\end{array}\right) .
\end{aligned}
$$

The fermion mass terms then become

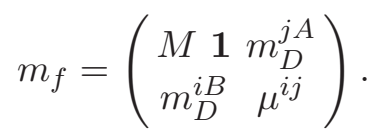


The one-loop corrections from translating from $\overline{\mathrm{MS}}$ to $\overline{\mathrm{DR}}^{\prime}$ only modify the fermionic part. Specialising to the case of interest, where ' $a$ ' is a singlet index, the corrections to the couplings are [47]:

$$
\begin{aligned}
\mu & \rightarrow \mu+\frac{g^{2}}{32 \pi^{2}}\left[C_{2}(i)+C_{2}(j)\right] \mu_{i j}=\frac{g^{2}}{32 \pi^{2}}\left\{C_{2}, \mu\right\} \\
M & \rightarrow M+\frac{g^{2}}{16 \pi^{2}} C_{2}(G) M=\frac{g^{2}}{32 \pi^{2}}\left\{C_{2}, M\right\} \\
Y_{a}^{\dagger} & \rightarrow Y_{a}^{\dagger}\left[1+\frac{g^{2}}{32 \pi^{2}}\left[C_{2}(j)+C_{2}(k)\right]\right] \\
& =Y_{a}^{\dagger}+\frac{g^{2}}{32 \pi^{2}}\left\{C_{2}, Y_{a}^{\dagger}\right\} \\
Y_{a} & \rightarrow 0 .
\end{aligned}
$$

For general Yukawa couplings (not involving a singlet) there are additional contributions; there is also a shift for general quartic couplings. However, they will not be relevant here.

For the Dirac gaugino mass, there is a similar transformation derived via the same technique:

$$
m_{D i A} \rightarrow m_{D i A}+\frac{g^{2}}{16 \pi^{2}} C_{2}(G) m_{D i A}=m_{D i A}+\frac{g^{2}}{32 \pi^{2}}\left\{C_{2}, m_{D i A}\right\}
$$

and hence

$$
m_{f} \rightarrow m_{f}+\frac{g^{2}}{32 \pi^{2}}\left\{C_{2}, m_{f}\right\}
$$

Note that there is no difference for the scalar trilinear couplings $r^{i j}{ }_{k}, r_{i j}{ }^{k}$ between $\overline{\mathrm{MS}}$ and $\overline{\mathrm{DR}}^{\prime}$, just as there is none for the couplings $A^{i j k}$.

\section{$2.3 \quad$ Result}

The expressions can now be transformed to the SUSY basis and the shifts applied as described in subsection 2.2 to the expressions for the non-SUSY tadpole RGEs in subsection 2.1 to obtain the RGEs for the tadpole in the theory described by equations (1.1), (1.2) and (1.4). After a large amount of tedious algebra, the result for the tadpole beta-functions can be simply written as

$$
\beta_{t^{a}}^{(i)} \equiv X_{S}^{(i)}+X_{\xi}^{(i)}+X_{D}^{(i)}
$$

where $i$ is the loop order, $X_{S}^{(i)}$ is the tadpole beta-function involving only standard soft terms, given by [39]

$$
\begin{aligned}
(4 \pi)^{2} X_{S}^{(1)}= & (4 \pi)^{2}\left(\gamma^{(1)}\right)_{b}^{a} t^{b} \\
& +A^{a e f}\left(B_{e f}+Y_{e f g} L^{g}\right)+Y_{e f h} \mu^{a h} B^{e f}+2 Y^{a j k} \mu_{j m}\left(m^{2}\right)_{k}^{m}
\end{aligned}
$$


and

$$
\begin{aligned}
(4 \pi)^{4} X_{S}^{(2)}= & (4 \pi)^{4}\left(\gamma^{(2)}\right)_{b}^{a} t^{b} \\
& -A^{a e f} A_{e g h} Y^{g h m} \mu_{m f}-Y^{a e m} \mu_{m f} A_{e g h} A^{f g h}-Y_{e f m} \mu^{m a} A^{f g h} Y_{g h n} \mu^{n e} \\
& -4 g^{2} A^{a e f}\left(C_{2}\right)_{f}^{m} \bar{M} \mu_{m e}-4 g^{2} Y_{e f p} \mu^{a p}\left(C_{2}\right)_{k}^{e} \bar{M} \mu^{k f}+8 g^{2}|M|^{2}\left(C_{2}\right)_{k}^{j} \mu_{j l} Y^{a k l} \\
& -4 g^{2} M\left(B_{e f}+Y_{e f p} L^{p}\right)\left(C_{2}\right)_{j}^{e} Y^{a j f} \\
& -Y^{a e m}\left(Y_{2}\right)_{m}^{k}\left(m^{2}\right)_{e}^{f} \mu_{k f}-2 Y^{a h m} Y_{m e g} Y^{f g n} \mu_{n h}\left(m^{2}\right)_{f}^{e} \\
& +\left(4 g^{2} C_{2}-Y_{2}\right)_{g}^{f}\left[Y^{a e k} \mu_{k f}\left(m^{2}\right)_{e}^{g}+Y^{a g k} \mu_{k e}\left(m^{2}\right)_{f}^{e}\right] \\
& +\left(4 g^{2} C_{2}-Y_{2}\right)_{g}^{f}\left[A^{a e g}\left(B_{e f}+Y_{e f m} L^{m}\right)+Y_{e f k} \mu^{k a} B^{e g}\right] \\
& -Y^{a e m} Y_{m g h}\left(B_{e f}+Y_{e f k} L^{k}\right) A^{f g h}
\end{aligned}
$$

In the above, $\left(\gamma^{(1)}\right)_{b}^{a}$ and $\left(\gamma^{(2)}\right)_{b}^{a}$ are the one- and two-loop chiral superfield anomalous dimensions for singlets respectively, given by

$$
\begin{aligned}
(4 \pi)^{2}\left(\gamma^{(1)}\right)_{b}^{a} & =\frac{1}{2}\left(Y_{2}\right)_{b}^{a}-2 g^{2}\left(C_{2}\right)_{b}^{a} \\
(4 \pi)^{4}\left(\gamma^{(2)}\right)_{b}^{a} & =2 g^{2}\left(C_{2}\right)_{k}^{l} Y^{a j k} Y_{b j l}-\frac{1}{2} Y_{b m n}\left(Y_{2}\right)_{r}^{n} Y^{m r a} .
\end{aligned}
$$

The new terms are

$$
\begin{aligned}
& (4 \pi)^{2} X_{\xi}^{(1)}=2 \sqrt{2} g_{Y} m_{D}^{a Y} \operatorname{Tr}\left(\mathcal{Y} m^{2}\right) \\
& (4 \pi)^{4} X_{\xi}^{(2)}=2 \sqrt{2} g_{Y} m_{D}^{a Y} \operatorname{Tr}\left(\mathcal{Y} m^{2}\left(4 g^{2} C_{2}-Y_{2}\right)\right)
\end{aligned}
$$

where $\mathcal{Y}$ is the charge operator of $\mathrm{U}(1)_{Y}$, and

$$
\begin{aligned}
(4 \pi)^{2} X_{D}^{(1)} & =2\left[\left(m_{D}^{2}\right)_{e f}\left(A^{a e f}+M Y^{a e f}\right)+Y_{e f k} \mu^{k a}\left(m_{D}^{2}\right)^{e f}\right] \\
(4 \pi)^{4} X_{D}^{(2)} & =4\left(\beta_{m_{D}}^{(1)} / m_{D}\right)_{g}^{f}\left[\left(m_{D}^{2}\right)_{e f}\left(A^{a e g}+M Y^{a e g}\right)+Y_{e f k} \mu^{k a}\left(m_{D}^{2}\right)^{e g}\right] \\
\left(\beta_{m_{D}}^{(1)} / m_{D}\right)_{g}^{f} & \equiv \frac{1}{2}\left(Y_{2}\right)_{g}^{f}+g^{2}\left(S_{2}-5 C_{2}(G)\right) \delta_{g}^{f} .
\end{aligned}
$$

The new contributions can be explained as follows. Firstly, the presence of $X_{\xi}$ is simply due to the renormalisation of the Fayet-Iliopoulos term. Since the FI term is absorbed as it is generated by the running into the soft term and the tadpole (so $\xi=0$ ), via equation (1.6), it is found that

$$
X_{\xi}=\frac{d}{d \log \mu}\left(\sqrt{2} m_{D}^{a Y} \xi_{Y}\right)=\sqrt{2} m_{D}^{a Y} \beta_{\xi_{Y}} .
$$

The result for the RGE of the FI term is then exactly as found in [48], without any additional piece coming from Dirac gaugino terms. They gave

$$
\begin{aligned}
16 \pi^{2} \hat{\beta}_{\xi}^{(1)} & =2 g \operatorname{Tr}\left[\mathcal{Y} m^{2}\right] \\
16 \pi^{2} \hat{\beta}_{\xi}^{(2)} & =-4 g \operatorname{Tr}\left[\mathcal{Y} m^{2} \gamma^{(1)}\right] \\
16 \pi^{2}\left(\gamma^{(1)}\right)_{i}^{j} & =\frac{1}{2}\left(Y_{2}\right)_{i}^{j}-2 g^{2}\left(C_{2}\right)_{i}^{j}
\end{aligned}
$$

which clearly exactly agrees with the above. 
Secondly, the $X_{D}$ terms contain two terms mimicking $B$ insertions - via equation (1.8) - in the supergraph when they do not involve a gauge line, but not $\mathrm{m}^{2}$ insertions. The additional term proportional to $M\left(m_{D}\right)^{2}$ is new and could not be obtained from shifting a standard term. Then the two-loop terms involve just wavefunction renormalisation of these.

Since the tree-level RGE for the tadpole only includes a gauge coupling via the FI term $X_{\xi}$, the two-loop contributions $X_{S}^{(2)}, X_{D}^{(2)}$ include only one gauge coupling, and so the interpretation of the above result for several gauge groups is straightforward; indeed if there is more than one $\mathrm{U}(1)$ gauge group then kinetic mixing can be easily included following the recipe of [49].

\section{RGEs of a Dirac gaugino extension of the (N)MSSM}

Here I present the full two-loop RGEs for a Dirac gaugino extension of the (N)MSSM. For generality, both Dirac and Majorana masses are included, as are all of the phenomenologically interesting superpotential couplings involving the new adjoint superfields, even those that break R-symmetry (this is motivated by generality and the possiblity of generating $\mu$ and $B_{\mu}$ terms [21]). Hence the model encompasses those studied in e.g. [4, 5] and [13, 16, 21] (the one-loop RGEs were presented in [16] without including Majorana gaugino masses).

The particle content of the model is just that of the MSSM extended by adjoint chiral superfields $\mathbf{S}, \mathbf{T}, \mathbf{O}$ where $S$ is a singlet, $T=\frac{1}{2}\left(\begin{array}{cc}T^{0} & \sqrt{2} T^{+} \\ \sqrt{2} T^{-} & -T^{0}\end{array}\right)$ an $\mathrm{SU}(2)$ triplet, and $\mathbf{O}$ a colour octet. The Dirac gaugino masses are denoted $m_{D 1}, m_{D 2}, m_{D 3}$ coupling the respective singlet, triplet and octet fermions to the gauginos of the corresponding group, with gauge couplings $g_{1}, g_{2}, g_{3}$ and Majorana masses $M_{1}, M_{2}, M_{3}$.

The superpotential of the model is:

$$
\begin{aligned}
W= & Y_{u} \hat{u} \hat{q} H_{u}-Y_{d} \hat{d} \hat{q} H_{d}-Y_{e} \hat{e} \hat{l} H_{d}+\mu \mathbf{H}_{\mathbf{u}} \cdot \mathbf{H}_{\mathbf{d}} \\
& +\lambda_{S} \mathbf{S H}_{\mathbf{u}} \cdot \mathbf{H}_{\mathbf{d}}+2 \lambda_{T} \mathbf{H}_{\mathbf{d}} \cdot \mathbf{T} \mathbf{H}_{\mathbf{u}} \\
& +L \mathbf{S}+\frac{M_{S}}{2} \mathbf{S}^{2}+\frac{\kappa}{3} \mathbf{S}^{3}+M_{T} \operatorname{tr}(\mathbf{T T})+M_{O} \operatorname{tr}(\mathbf{O O}),
\end{aligned}
$$

the usual scalar soft terms are

$$
\begin{aligned}
-\Delta \mathcal{L}_{\mathrm{MSSM}}^{\text {scalar soft }}= & {\left[A_{u} \hat{u} \hat{q} H_{u}-A_{d} \hat{d} \hat{q} H_{d}-A_{e} \hat{e} \hat{l} H_{d}+h . c .\right] } \\
& +m_{H_{u}}^{2}\left|H_{u}\right|^{2}+m_{H_{d}}^{2}\left|H_{d}\right|^{2}+\left[B_{\mu} H_{u} \cdot H_{d}+h . c .\right] \\
& +\hat{q}^{i}\left(m_{q}^{2}\right)_{i}^{j} \hat{q}_{j}+\hat{u}^{i}\left(m_{u}^{2}\right)_{i}^{j} \hat{u}_{j}+\hat{d}^{i}\left(m_{d}^{2}\right)_{i}^{j} \hat{d}_{j}+\hat{l}^{i}\left(m_{l}^{2}\right)_{i}^{j} \hat{l}_{j}+\hat{e}^{i}\left(m_{e}^{2}\right)_{i}^{j} \hat{e}_{j}
\end{aligned}
$$

and there are soft terms involving the adjoint scalars

$$
\begin{aligned}
-\Delta \mathcal{L}_{\text {adjoints }}^{\text {scalar soft }}= & \left(t_{S} S+\text { h.c. }\right) \\
& +m_{S}^{2}|S|^{2}+\frac{1}{2} B_{S}\left(S^{2}+\text { h.c. }\right)+2 m_{T}^{2} \operatorname{tr}\left(T^{\dagger} T\right)+\left(B_{T} \operatorname{tr}(T T)+\text { h.c. }\right) \\
& +\left[A_{S} \lambda_{S} S H_{u} \cdot H_{d}+2 A_{T} H_{d} \cdot T H_{u}+\frac{1}{3} \kappa A_{\kappa} S^{3}+\text { h.c. }\right] \\
& +2 m_{O}^{2} \operatorname{tr}\left(O^{\dagger} O\right)+\left(B_{O} \operatorname{tr}(O O)+\text { h.c. }\right)
\end{aligned}
$$

with the definition $H_{u} \cdot H_{d}=H_{u}^{+} H_{d}^{-}-H_{u}^{0} H_{d}^{0}$. 
The most general renormalisable Lagrangian would include additional superpotential interactions $^{1}$

$$
W_{2}=\lambda_{S T} \mathbf{S t r}(\mathbf{T T})+\lambda_{S O} \mathbf{S t r}(\mathbf{O O})+\frac{\kappa_{O}}{3} \operatorname{tr}(\mathbf{O O O}) .
$$

as well as the equivalent adjoint scalar A-terms. It would be straightforward to add these (and indeed $\lambda_{S T}, \lambda_{S O}$ would contribute to the singlet tadpole) but they violate R-symmetry (admittedly as do all the terms on the last line of equation (3.1)) and, more importantly, are much less phenomenologically interesting than the other terms.

To determine the parameters of the model, the standard soft terms and Dirac gaugino masses can be run according to the RGEs given below, and then at the scale of interest determine the physical soft masses by applying the shifts

$$
\begin{aligned}
m_{S}^{2} & \rightarrow m_{S}^{2}+2\left|m_{D 1}\right|^{2} \\
B_{S}^{2} & \rightarrow B_{S}^{2}+2 m_{D 1}^{2}
\end{aligned}
$$

and similarly for $m_{T}, B_{T}, m_{O}, B_{O}$.

The standard soft and supersymmetric RGEs, presented in appendix B, were calculated by implementing the model in SARAH $[50,51]$ version 3.0.41. ${ }^{2}$ The RGEs for the Dirac mass terms and tadpoles are presented below, using the results of the previous section and the anomalous dimensions of the adjoint superfields.

\subsection{Dirac gaugino masses}

Note $g_{Y}=\sqrt{\frac{3}{5}} g_{1} ;$ then

$$
\begin{aligned}
(4 \pi)^{2} \beta_{m_{D 1}}^{(1)}= & m_{D 1}\left[2\left(\left|\kappa_{S}\right|^{2}+\left|\lambda_{S}\right|^{2}\right)+\frac{33}{5} g_{1}^{2}\right] \\
(4 \pi)^{4} \beta_{m_{D 1}}^{(2)}= & m_{D 1}\left[-8\left|\kappa_{S}\right|^{4}-8\left|\lambda_{S}\right|^{2}\left|\kappa_{S}\right|^{2}\right. \\
& -\frac{2}{5}\left|\lambda_{S}\right|^{2}\left(10\left|\lambda_{S}\right|^{2}-15 g_{2}^{2}+15 \operatorname{Tr}\left(Y_{d} Y_{d}^{\dagger}\right)+15 \operatorname{Tr}\left(Y_{u} Y_{u}^{\dagger}\right)+30\left|\lambda_{T}\right|^{2}+5 \operatorname{Tr}\left(Y_{e} Y_{e}^{\dagger}\right)\right) \\
& +\frac{1}{25} g_{1}^{2}\left(-130 \operatorname{Tr}\left(Y_{u} Y_{u}^{\dagger}\right)+135 g_{2}^{2}+199 g_{1}^{2}+440 g_{3}^{2}-70 \operatorname{Tr}\left(Y_{d} Y_{d}^{\dagger}\right)\right. \\
& \left.\left.-90\left|\lambda_{T}\right|^{2}-90 \operatorname{Tr}\left(Y_{e} Y_{e}^{\dagger}\right)\right)\right] \\
(4 \pi)^{2} \beta_{m_{D 2}}^{(1)}= & m_{D 2}\left[2\left|\lambda_{T}\right|^{2}-g_{2}^{2}\right] \\
(4 \pi)^{4} \beta_{m_{D 2}}^{(2)}= & m_{D 2}\left[28 g_{2}^{4}-12\left|\lambda_{T}\right|^{4}\right. \\
& +\frac{2}{5}\left|\lambda_{T}\right|^{2}\left(-10\left|\lambda_{S}\right|^{2}-15 \operatorname{Tr}\left(Y_{d} Y_{d}^{\dagger}\right)-15 \operatorname{Tr}\left(Y_{u} Y_{u}^{\dagger}\right)+3 g_{1}^{2}-5 g_{2}^{2}-5 \operatorname{Tr}\left(Y_{e} Y_{e}^{\dagger}\right)\right) \\
& +\frac{1}{5} g_{2}^{2}\left(-10\left|\lambda_{S}\right|^{2}-10 \operatorname{Tr}\left(Y_{e} Y_{e}^{\dagger}\right)+120 g_{3}^{2}+245 g_{2}^{2}-30 \operatorname{Tr}\left(Y_{d} Y_{d}^{\dagger}\right)\right. \\
& \left.\left.-30 \operatorname{Tr}\left(Y_{u} Y_{u}^{\dagger}\right)-70\left|\lambda_{T}\right|^{2}+9 g_{1}^{2}\right)\right]
\end{aligned}
$$

\footnotetext{
${ }^{1}$ Note there are no terms $\operatorname{tr}(\mathbf{T}), \operatorname{tr}(\mathbf{O}), \operatorname{tr}(\mathbf{T T T})$ since these vanish by gauge invariance.
}

${ }^{2}$ The results from this paper have now been implemented in version 3.2.0. 


$$
\begin{aligned}
& (4 \pi)^{2} \beta_{m_{D 3}}^{(1)}=m_{D 3}\left[-6 g_{3}^{2}\right] \\
& (4 \pi)^{4} \beta_{m_{D 3}}^{(2)}=m_{D 3}\left[36 g_{3}^{4}+\frac{1}{5} g_{3}^{2}\left(11 g_{1}^{2}-20 \operatorname{Tr}\left(Y_{d} Y_{d}^{\dagger}\right)-20 \operatorname{Tr}\left(Y_{u} Y_{u}^{\dagger}\right)+340 g_{3}^{2}+45 g_{2}^{2}\right)\right]
\end{aligned}
$$

\subsection{Tadpole equation}

The one-loop contribution to $X_{S}$ is given by

$$
\begin{aligned}
(4 \pi)^{2} X_{S}^{(1)}= & \left(2\left|\kappa_{S}\right|^{2}+2\left|\lambda_{S}\right|^{2}\right) t_{S} \\
& +4 m_{S}^{2} \kappa_{S} M_{S}^{*}+2 M_{S} B_{S} \kappa_{S}^{*}+4 M_{S} B_{\mu} \lambda_{S}^{*}+4 \lambda_{S} \mu^{*}\left(m_{H_{d}}^{2}+m_{H_{u}}^{2}\right) \\
& +4 L_{S} \kappa_{S}^{*} A_{\kappa}+2 B_{S}^{*} A_{\kappa}+4 L_{S} \lambda_{S}^{*} A_{S}+4 B_{\mu}^{*} A_{S} .
\end{aligned}
$$

The two-loop contribution is much longer and is given in the appendix, equation (B.76).

For the Dirac gaugino contribution arising via the FI term define

$$
\begin{aligned}
\sigma_{1,1} & \equiv \sqrt{\frac{3}{5}} g_{1} \operatorname{Tr}\left(\mathcal{Y} m^{2}\right) \\
\sigma_{3,1} & \equiv \frac{1}{4} \sqrt{\frac{3}{5}} g_{1} \operatorname{Tr}\left(\mathcal{Y} m^{2}\left(4 g^{2} C_{2}-Y_{2}\right)\right) .
\end{aligned}
$$

$\sigma_{1,1}$ and $\sigma_{3,1}$ also appear in the scalar mass RGEs; the full expressions for these in this model are given in appendix equation (B.77). Then

$$
\begin{aligned}
& (4 \pi)^{2} X_{\xi}^{(1)}=2 \sqrt{2} m_{D 1} \sigma_{1,1} \\
& (4 \pi)^{4} X_{\xi}^{(2)}=8 \sqrt{2} m_{D 1} \sigma_{3,1} .
\end{aligned}
$$

Finally, the Dirac gaugino parts are given by

$$
\begin{aligned}
& (4 \pi)^{2} X_{D}^{(1)}=4\left(m_{D 1}^{*}\right)^{2}\left[A_{\kappa}+M_{1} \kappa_{S}\right]+4 m_{D 1}^{2} \kappa_{S}^{*} M_{S} \\
& (4 \pi)^{4} X_{D}^{(2)}=8\left[2\left(\left|\kappa_{S}\right|^{2}+\left|\lambda_{S}\right|^{2}\right)+\frac{33}{5} g_{1}^{2}\right]\left[\left(m_{D 1}^{*}\right)^{2}\left(A_{\kappa}+M_{1} \kappa_{S}\right)+m_{D 1}^{2} \kappa_{S}^{*} M_{S}\right] .
\end{aligned}
$$

The conclusion is that for R-symmetric soft terms that obey $\operatorname{Tr}\left(\mathcal{Y} m^{2}\right)=0$ at some scale (such as, for example, in gauge mediation with R-symmetric F-terms [10-12]), the new Dirac gaugino mass-dependent contributions to the singlet tadpole will be safely negligible.

\section{Conclusions}

The set of two-loop RGEs for Dirac gaugino models is now complete, which opens up the possibility of implementing them in spectrum generators (such as by an extension of $[50,51])$ and studying their precision phenomenology. Since Dirac mass terms only appear in supersoft operators (in spontaneously broken supersymmetric theories) their effect upon the RGEs is extremely mild. In particular, they remain on an RG invariant trajectory with respect to the mass squared and $B$ terms, but the result of this paper is that, although they contribute new terms to the tadpole RGE, these are exhausted at one loop and the two-loop correction is merely a particular form of wavefunction renormalisation of the one-loop term. 
As mentioned in the introduction, the tadpole RGE is particularly important for studying the Higgs potential, and since the new Higgs couplings in Dirac gaugino models can allow a natural increase in the Higgs mass - alleviating fine-tuning - it is now imperative to study the Higgs sector of Dirac gaugino models including full loop corrections $[52,53]$.

\section{Acknowledgments}

I was supported by ERC advanced grant 226371. I thank I. Jack and D.R.T. Jones for helpful discussions. I would also like to thank the Isaac Newton Institute, and the organisers of the string phenomenology workshop in the "Mathematics and Applications of Branes in String and M-theory" programme, for excellent hospitality and providing a stimulating environment while this project was completed. Finally I thank K.A. West for careful reading of the manuscript, and F. Staub for comments on the first version.

\section{A General non-supersoft operators}

This paper has focussed on the contribution of supersoft operators to the tadpole RGE, but it is natural to ask what would happen if we included terms that do not fall under this category; in particular, these could be present in the NMSSM. Let us suppose that we include "hard" terms of the form

$$
\mathcal{L} \supset-m_{D(H)}^{i A} \psi_{i} \lambda_{A}-\frac{1}{2} r_{(H) k}^{i j} \phi_{i} \phi_{j} \phi^{k}+\text { h.c. }
$$

We can write these terms in superspace as

$$
-\int d^{4} \theta \eta \bar{\eta}\left[m_{D(H)}^{i A}\left(D^{\alpha} \Phi_{i}\right) W_{\alpha}+\frac{1}{2} r_{(H) k}^{i j} \Phi_{i} \Phi_{j} \Phi^{k}+\text { h.c. }\right]
$$

where $\eta$ is a spurion, and since (allowing for the two from each $\Phi$ insertion and $\bar{D}^{2} D_{\alpha}$ from the $W_{\alpha}$ ) each vertex leads to six $D_{\alpha}, \bar{D}_{\dot{\alpha}}$ insertions we expect that quadratic divergences will be generated at some order. This is easiest to see for any trilinear hard term coupling to the singlet scalar, e.g. $r_{(H) H_{u}}^{S H_{u}}$, where a tadpole loop (shown left in figure 1) gives a divergence proportional to $r_{(H) H_{u}}^{S H_{u}} \Lambda^{2}$ (note that the would-be quadratic divergences from supersoft terms cancel among themselves at one-loop order since in that case the total divergence is proportional to $\operatorname{Tr}(\mathcal{Y})$ ). However, more interesting terms, for example, are those considered in the context of the MSSM in [41]:

$$
\mathcal{L} \supset-m_{9} \bar{H}_{d} Q U-m_{7} \bar{H}_{u} Q D-m_{5} \bar{H}_{u} L E+\text { h.c. }
$$

Clearly the quadratic divergences do not appear at one-loop order, and by dimensional anaysis we know that they can only appear in amplitudes with Yukawa and gauge couplings (they appear in the effective action as $\propto r_{(H)} \Lambda^{2}, m_{D(H)} \Lambda^{2}$ ). Simply by writing down diagrams (see e.g. figure 1 right) we can establish that quadratic divergences can first appear for these terms at three loops. 

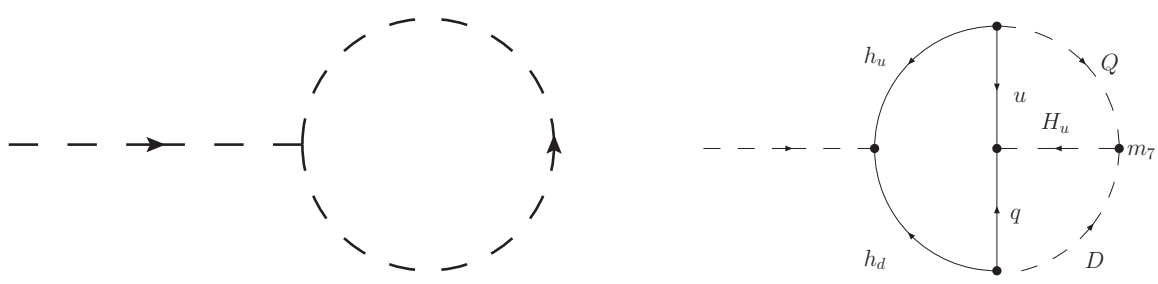

Figure 1. Two example tadpole graphs leading to quadratic divergences when the appropriate hard breaking terms are present. Left: when a hard breaking term coupling directly to the singlet scalar is present; right: for a term $m_{7} \bar{H}_{u} Q D$.

Finally, for the $m_{D(H)}^{i A}$ terms, we might naively think that the quadratic divergences first arrive at two loops, but it is straightforward to show that no non-vanishing operator can be written down at this order (e.g. $\left.g_{Y} Y_{a i j}\left(t^{A}\right)_{k}^{i} Y^{j k l} m_{D(H) l A}=0\right)$. However, we do expect them to appear at three-loop order (and can write down terms such as $\left.g_{Y} Y_{a i j}\left(t^{A}\right)_{k}^{i}\left(Y_{2}\right)_{l}^{k} Y^{j l m} m_{D(H) m A}\right)$ although it is beyond the scope of this work to calculate these divergent terms.

If, despite the presence of the quadratic divergences, we wanted to still write down the RGEs for the tadpole term up to two-loop order, then it is straightforward to do this from the non-supersymmetric expressions in section 2.1 and translate the couplings following section 2.2 but adding the additional terms to $h_{e f g}$ and $m_{f}$. Of most interest would be terms which do not lead to one-loop quadratic divergences, so $h_{a e f}=0$; if we added only the terms in equation (A.3) then the one-loop tadpole RGE equation would obviously not be changed at all, and only the terms $\beta_{t_{a}} \supset-\frac{g^{2}}{2} h_{a e f} h_{e g h} h_{f g h}-g^{2} m_{e f}^{2} \lambda_{a e g h} h_{f g h}$ would yield new contributions at two loops. However, this actually comprises 24 new terms that must be added, and so we neglect to present the full expressions here.

\section{B Two-loop RGEs for SUSY and standard soft terms}

In this appendix the RGEs for the standard soft terms in the model of section 3 are presented. For brevity, the factors of $(4 \pi)^{2}$ and $(4 \pi)^{4}$ for one- and two-loop quantities shall be dropped. All of the below were generated using SARAH [50, 51] version 3.0.41 and so are presented in the notation of that package.

\section{B.1 Anomalous dimensions}

$$
\begin{aligned}
\gamma_{\hat{q}}^{(1)}= & -\frac{1}{30}\left(45 g_{2}^{2}+80 g_{3}^{2}+g_{1}^{2}\right) \mathbf{1}+Y_{d}^{\dagger} Y_{d}+Y_{u}^{\dagger} Y_{u} \\
\gamma_{\hat{q}}^{(2)}= & +\left(8 g_{2}^{2} g_{3}^{2}+\frac{1}{90} g_{1}^{2}\left(16 g_{3}^{2}+9 g_{2}^{2}\right)+\frac{199}{900} g_{1}^{4}+\frac{27}{4} g_{2}^{4}+\frac{64}{9} g_{3}^{4}\right) \mathbf{1}+\frac{4}{5} g_{1}^{2} Y_{u}^{\dagger} Y_{u}-\left|\lambda_{S}\right|^{2} Y_{u}^{\dagger} Y_{u} \\
& -3\left|\lambda_{T}\right|^{2} Y_{u}^{\dagger} Y_{u}-2 Y_{d}^{\dagger} Y_{d} Y_{d}^{\dagger} Y_{d}-2 Y_{u}^{\dagger} Y_{u} Y_{u}^{\dagger} Y_{u} \\
& +Y_{d}^{\dagger} Y_{d}\left(-3\left|\lambda_{T}\right|^{2}-3 \operatorname{Tr}\left(Y_{d} Y_{d}^{\dagger}\right)+\frac{2}{5} g_{1}^{2}-\left|\lambda_{S}\right|^{2}-\operatorname{Tr}\left(Y_{e} Y_{e}^{\dagger}\right)\right)-3 Y_{u}^{\dagger} Y_{u} \operatorname{Tr}\left(Y_{u} Y_{u}^{\dagger}\right)
\end{aligned}
$$




$$
\begin{aligned}
& \gamma_{\hat{l}}^{(1)}=-\frac{3}{10}\left(5 g_{2}^{2}+g_{1}^{2}\right) \mathbf{1}+Y_{e}^{\dagger} Y_{e} \\
& \gamma_{\hat{l}}^{(2)}=+\frac{9}{100}\left(10 g_{1}^{2} g_{2}^{2}+23 g_{1}^{4}+75 g_{2}^{4}\right) \mathbf{1}-2 Y_{e}^{\dagger} Y_{e} Y_{e}^{\dagger} Y_{e} \\
& +Y_{e}^{\dagger} Y_{e}\left(-3\left|\lambda_{T}\right|^{2}-3 \operatorname{Tr}\left(Y_{d} Y_{d}^{\dagger}\right)+\frac{6}{5} g_{1}^{2}-\left|\lambda_{S}\right|^{2}-\operatorname{Tr}\left(Y_{e} Y_{e}^{\dagger}\right)\right) \\
& \gamma_{\hat{H}_{d}}^{(1)}=3\left|\lambda_{T}\right|^{2}+3 \operatorname{Tr}\left(Y_{d} Y_{d}^{\dagger}\right)-\frac{3}{10} g_{1}^{2}-\frac{3}{2} g_{2}^{2}+\left|\lambda_{S}\right|^{2}+\operatorname{Tr}\left(Y_{e} Y_{e}^{\dagger}\right) \\
& \gamma_{\hat{H}_{d}}^{(2)}=+\frac{207}{100} g_{1}^{4}+\frac{9}{10} g_{1}^{2} g_{2}^{2}+\frac{27}{4} g_{2}^{4}+12 g_{2}^{2}\left|\lambda_{T}\right|^{2}-2 \lambda_{S}\left|\kappa_{S}\right|^{2} \lambda_{S}^{*}-3 \lambda_{S}^{2} \lambda_{S}^{*, 2}-15 \lambda_{T}^{2} \lambda_{T}^{*, 2} \\
& -\frac{2}{5} g_{1}^{2} \operatorname{Tr}\left(Y_{d} Y_{d}^{\dagger}\right)+16 g_{3}^{2} \operatorname{Tr}\left(Y_{d} Y_{d}^{\dagger}\right)+\frac{6}{5} g_{1}^{2} \operatorname{Tr}\left(Y_{e} Y_{e}^{\dagger}\right)-9\left|\lambda_{T}\right|^{2} \operatorname{Tr}\left(Y_{u} Y_{u}^{\dagger}\right) \\
& -3\left|\lambda_{S}\right|^{2}\left(2 \lambda_{T} \lambda_{T}^{*}+\operatorname{Tr}\left(Y_{u} Y_{u}^{\dagger}\right)\right)-9 \operatorname{Tr}\left(Y_{d} Y_{d}^{\dagger} Y_{d} Y_{d}^{\dagger}\right)-3 \operatorname{Tr}\left(Y_{d} Y_{u}^{\dagger} Y_{u} Y_{d}^{\dagger}\right) \\
& -3 \operatorname{Tr}\left(Y_{e} Y_{e}^{\dagger} Y_{e} Y_{e}^{\dagger}\right) \\
& \gamma_{\hat{H}_{u}}^{(1)}=3\left|\lambda_{T}\right|^{2}-\frac{3}{10}\left(-10 \operatorname{Tr}\left(Y_{u} Y_{u}^{\dagger}\right)+5 g_{2}^{2}+g_{1}^{2}\right)+\left|\lambda_{S}\right|^{2} \\
& \gamma_{\hat{H}_{u}}^{(2)}=+\frac{207}{100} g_{1}^{4}+\frac{9}{10} g_{1}^{2} g_{2}^{2}+\frac{27}{4} g_{2}^{4}+12 g_{2}^{2}\left|\lambda_{T}\right|^{2}-2 \lambda_{S}\left|\kappa_{S}\right|^{2} \lambda_{S}^{*}-3 \lambda_{S}^{2} \lambda_{S}^{*, 2}-15 \lambda_{T}^{2} \lambda_{T}^{* 2} \\
& -9\left|\lambda_{T}\right|^{2} \operatorname{Tr}\left(Y_{d} Y_{d}^{\dagger}\right)-3\left|\lambda_{T}\right|^{2} \operatorname{Tr}\left(Y_{e} Y_{e}^{\dagger}\right)-\left|\lambda_{S}\right|^{2}\left(3 \operatorname{Tr}\left(Y_{d} Y_{d}^{\dagger}\right)+6 \lambda_{T} \lambda_{T}^{*}+\operatorname{Tr}\left(Y_{e} Y_{e}^{\dagger}\right)\right) \\
& +\frac{4}{5} g_{1}^{2} \operatorname{Tr}\left(Y_{u} Y_{u}^{\dagger}\right)+16 g_{3}^{2} \operatorname{Tr}\left(Y_{u} Y_{u}^{\dagger}\right)-3 \operatorname{Tr}\left(Y_{d} Y_{u}^{\dagger} Y_{u} Y_{d}^{\dagger}\right)-9 \operatorname{Tr}\left(Y_{u} Y_{u}^{\dagger} Y_{u} Y_{u}^{\dagger}\right) \\
& \gamma_{\hat{d}}^{(1)}=2 Y_{d}^{*} Y_{d}^{T}-\frac{2}{15}\left(20 g_{3}^{2}+g_{1}^{2}\right) \mathbf{1} \\
& \gamma_{\hat{d}}^{(2)}=+\frac{2}{225}\left(101 g_{1}^{4}+800 g_{3}^{4}+80 g_{1}^{2} g_{3}^{2}\right) \mathbf{1}-2\left(Y_{d}^{*} Y_{d}^{T} Y_{d}^{*} Y_{d}^{T}+Y_{d}^{*} Y_{u}^{T} Y_{u}^{*} Y_{d}^{T}\right) \\
& +Y_{d}^{*} Y_{d}^{T}\left(-2\left|\lambda_{S}\right|^{2}-2 \operatorname{Tr}\left(Y_{e} Y_{e}^{\dagger}\right)+6 g_{2}^{2}-6\left|\lambda_{T}\right|^{2}-6 \operatorname{Tr}\left(Y_{d} Y_{d}^{\dagger}\right)+\frac{2}{5} g_{1}^{2}\right) \\
& \gamma_{\hat{u}}^{(1)}=2 Y_{u}^{*} Y_{u}^{T}-\frac{8}{15}\left(5 g_{3}^{2}+g_{1}^{2}\right) \mathbf{1} \\
& \gamma_{\hat{u}}^{(2)}=+\frac{8}{225}\left(107 g_{1}^{4}+200 g_{3}^{4}+80 g_{1}^{2} g_{3}^{2}\right) \mathbf{1} \\
& -\frac{2}{5}\left(5\left(Y_{u}^{*} Y_{d}^{T} Y_{d}^{*} Y_{u}^{T}+Y_{u}^{*} Y_{u}^{T} Y_{u}^{*} Y_{u}^{T}\right)\right. \\
& \left.+Y_{u}^{*} Y_{u}^{T}\left(-15 g_{2}^{2}+15\left|\lambda_{T}\right|^{2}+15 \operatorname{Tr}\left(Y_{u} Y_{u}^{\dagger}\right)+5\left|\lambda_{S}\right|^{2}+g_{1}^{2}\right)\right) \\
& \gamma_{\hat{e}}^{(1)}=2 Y_{e}^{*} Y_{e}^{T}-\frac{6}{5} g_{1}^{2} \mathbf{1} \\
& \gamma_{\hat{e}}^{(2)}=+\frac{234}{25} g_{1}^{4} \mathbf{1}-2 Y_{e}^{*} Y_{e}^{T} Y_{e}^{*} Y_{e}^{T} \\
& +Y_{e}^{*} Y_{e}^{T}\left(-2\left|\lambda_{S}\right|^{2}-2 \operatorname{Tr}\left(Y_{e} Y_{e}^{\dagger}\right)+6 g_{2}^{2}-6\left|\lambda_{T}\right|^{2}-6 \operatorname{Tr}\left(Y_{d} Y_{d}^{\dagger}\right)-\frac{6}{5} g_{1}^{2}\right) \\
& \gamma_{S}^{(1)}=2\left(\left|\kappa_{S}\right|^{2}+\left|\lambda_{S}\right|^{2}\right) \\
& \gamma_{S}^{(2)}=-8 \kappa_{S}^{2} \kappa_{S}^{*, 2}-8 \lambda_{S}\left|\kappa_{S}\right|^{2} \lambda_{S}^{*} \\
& -\frac{2}{5}\left|\lambda_{S}\right|^{2}\left(10 \lambda_{S} \lambda_{S}^{*}-15 g_{2}^{2}+15 \operatorname{Tr}\left(Y_{d} Y_{d}^{\dagger}\right)+15 \operatorname{Tr}\left(Y_{u} Y_{u}^{\dagger}\right)+30 \lambda_{T} \lambda_{T}^{*}-3 g_{1}^{2}+5 \operatorname{Tr}\left(Y_{e} Y_{e}^{\dagger}\right)\right)
\end{aligned}
$$




$$
\begin{aligned}
\gamma_{T}^{(1)}= & 2\left|\lambda_{T}\right|^{2}-4 g_{2}^{2} \\
\gamma_{T}^{(2)}= & +28 g_{2}^{4}-12 \lambda_{T}^{2} \lambda_{T}^{*, 2} \\
& +\frac{2}{5}\left|\lambda_{T}\right|^{2}\left(-10 \lambda_{S} \lambda_{S}^{*}-15 \operatorname{Tr}\left(Y_{d} Y_{d}^{\dagger}\right)-15 \operatorname{Tr}\left(Y_{u} Y_{u}^{\dagger}\right)+3 g_{1}^{2}-5 g_{2}^{2}-5 \operatorname{Tr}\left(Y_{e} Y_{e}^{\dagger}\right)\right) \\
\gamma_{O}^{(1)}= & -6 g_{3}^{2} \\
\gamma_{O}^{(2)}= & 36 g_{3}^{4}
\end{aligned}
$$

\section{B.2 Gauge couplings}

$$
\begin{aligned}
\beta_{g_{1}}^{(1)}= & \frac{33}{5} g_{1}^{3} \\
\beta_{g_{1}}^{(2)}= & \frac{1}{25} g_{1}^{3}\left(-130 \operatorname{Tr}\left(Y_{u} Y_{u}^{\dagger}\right)+135 g_{2}^{2}+199 g_{1}^{2}-30\left|\lambda_{S}\right|^{2}+440 g_{3}^{2}-70 \operatorname{Tr}\left(Y_{d} Y_{d}^{\dagger}\right)\right. \\
& \left.-90\left|\lambda_{T}\right|^{2}-90 \operatorname{Tr}\left(Y_{e} Y_{e}^{\dagger}\right)\right) \\
\beta_{g_{2}}^{(1)}= & 3 g_{2}^{3} \\
\beta_{g_{2}}^{(2)}= & \frac{1}{5} g_{2}^{3}\left(-10\left|\lambda_{S}\right|^{2}-10 \operatorname{Tr}\left(Y_{e} Y_{e}^{\dagger}\right)+120 g_{3}^{2}+245 g_{2}^{2}-30 \operatorname{Tr}\left(Y_{d} Y_{d}^{\dagger}\right)\right. \\
& \left.-30 \operatorname{Tr}\left(Y_{u} Y_{u}^{\dagger}\right)-70\left|\lambda_{T}\right|^{2}+9 g_{1}^{2}\right) \\
\beta_{g_{3}}^{(1)}= & 0 \\
\beta_{g_{3}}^{(2)}= & \frac{1}{5} g_{3}^{3}\left(11 g_{1}^{2}-20 \operatorname{Tr}\left(Y_{d} Y_{d}^{\dagger}\right)-20 \operatorname{Tr}\left(Y_{u} Y_{u}^{\dagger}\right)+340 g_{3}^{2}+45 g_{2}^{2}\right)
\end{aligned}
$$

\section{B.3 Gaugino mass parameters}

$$
\begin{aligned}
\beta_{M_{1}}^{(1)}= & \frac{66}{5} g_{1}^{2} M_{1} \\
\beta_{M_{1}}^{(2)}= & \frac{2}{25} g_{1}^{2}\left(398 g_{1}^{2} M_{1}+135 g_{2}^{2} M_{1}+440 g_{3}^{2} M_{1}+440 g_{3}^{2} M_{3}+135 g_{2}^{2} M_{2}\right. \\
& -30 \lambda_{S}^{*}\left(-A_{S}+M_{1} \lambda_{S}\right)-90 \lambda_{T}^{*}\left(-A_{T}+M_{1} \lambda_{T}\right) \\
& -70 M_{1} \operatorname{Tr}\left(Y_{d} Y_{d}^{\dagger}\right)-90 M_{1} \operatorname{Tr}\left(Y_{e} Y_{e}^{\dagger}\right)-130 M_{1} \operatorname{Tr}\left(Y_{u} Y_{u}^{\dagger}\right)+70 \operatorname{Tr}\left(Y_{d}^{\dagger} A_{d}\right)+90 \operatorname{Tr}\left(Y_{e}^{\dagger} A_{e}\right) \\
& \left.+130 \operatorname{Tr}\left(Y_{u}^{\dagger} A_{u}\right)\right) \\
\beta_{M_{2}}^{(1)}= & 6 g_{2}^{2} M_{2} \\
\beta_{M_{2}}^{(2)}= & \frac{2}{5} g_{2}^{2}\left(9 g_{1}^{2} M_{1}+120 g_{3}^{2} M_{3}+9 g_{1}^{2} M_{2}+490 g_{2}^{2} M_{2}+120 g_{3}^{2} M_{2}\right. \\
& -10 \lambda_{S}^{*}\left(-A_{S}+M_{2} \lambda_{S}\right)-70 \lambda_{T}^{*}\left(-A_{T}+M_{2} \lambda_{T}\right) \\
& -30 M_{2} \operatorname{Tr}\left(Y_{d} Y_{d}^{\dagger}\right)-10 M_{2} \operatorname{Tr}\left(Y_{e} Y_{e}^{\dagger}\right)-30 M_{2} \operatorname{Tr}\left(Y_{u} Y_{u}^{\dagger}\right)+30 \operatorname{Tr}\left(Y_{d}^{\dagger} A_{d}\right)+10 \operatorname{Tr}\left(Y_{e}^{\dagger} A_{e}\right) \\
& \left.+30 \operatorname{Tr}\left(Y_{u}^{\dagger} A_{u}\right)\right) \\
\beta_{M_{3}}^{(1)}= & 0
\end{aligned}
$$




$$
\begin{aligned}
\beta_{M_{3}}^{(2)}= & \frac{2}{5} g_{3}^{2}\left(11 g_{1}^{2} M_{1}+11 g_{1}^{2} M_{3}+45 g_{2}^{2} M_{3}+680 g_{3}^{2} M_{3}+45 g_{2}^{2} M_{2}-20 M_{3} \operatorname{Tr}\left(Y_{d} Y_{d}^{\dagger}\right)\right. \\
& \left.-20 M_{3} \operatorname{Tr}\left(Y_{u} Y_{u}^{\dagger}\right)+20 \operatorname{Tr}\left(Y_{d}^{\dagger} A_{d}\right)+20 \operatorname{Tr}\left(Y_{u}^{\dagger} A_{u}\right)\right)
\end{aligned}
$$

\section{B.4 Trilinear superpotential parameters}

$$
\begin{aligned}
& \beta_{Y_{u}}^{(1)}=3 Y_{u} Y_{u}^{\dagger} Y_{u}+Y_{u}\left(-3 g_{2}^{2}+3\left|\lambda_{T}\right|^{2}+3 \operatorname{Tr}\left(Y_{u} Y_{u}^{\dagger}\right)-\frac{13}{15} g_{1}^{2}-\frac{16}{3} g_{3}^{2}+\left|\lambda_{S}\right|^{2}\right)+Y_{u} Y_{d}^{\dagger} Y_{d} \\
& \beta_{Y_{u}}^{(2)}=+\frac{2}{5} g_{1}^{2} Y_{u} Y_{u}^{\dagger} Y_{u}+6 g_{2}^{2} Y_{u} Y_{u}^{\dagger} Y_{u}-3\left|\lambda_{S}\right|^{2} Y_{u} Y_{u}^{\dagger} Y_{u}-9\left|\lambda_{T}\right|^{2} Y_{u} Y_{u}^{\dagger} Y_{u} \\
& -2 Y_{u} Y_{d}^{\dagger} Y_{d} Y_{d}^{\dagger} Y_{d}-2 Y_{u} Y_{d}^{\dagger} Y_{d} Y_{u}^{\dagger} Y_{u}-4 Y_{u} Y_{u}^{\dagger} Y_{u} Y_{u}^{\dagger} Y_{u} \\
& +Y_{u} Y_{d}^{\dagger} Y_{d}\left(-3\left|\lambda_{T}\right|^{2}-3 \operatorname{Tr}\left(Y_{d} Y_{d}^{\dagger}\right)+\frac{2}{5} g_{1}^{2}-\left|\lambda_{S}\right|^{2}-\operatorname{Tr}\left(Y_{e} Y_{e}^{\dagger}\right)\right) \\
& -9 Y_{u} Y_{u}^{\dagger} Y_{u} \operatorname{Tr}\left(Y_{u} Y_{u}^{\dagger}\right) \\
& +Y_{u}\left(\frac{2743}{450} g_{1}^{4}+g_{1}^{2} g_{2}^{2}+\frac{27}{2} g_{2}^{4}+\frac{136}{45} g_{1}^{2} g_{3}^{2}+8 g_{2}^{2} g_{3}^{2}+\frac{128}{9} g_{3}^{4}+12 g_{2}^{2}\left|\lambda_{T}\right|^{2}-2 \lambda_{S}\left|\kappa_{S}\right|^{2} \lambda_{S}^{*}\right. \\
& -3 \lambda_{S}^{2} \lambda_{S}^{*, 2}-15 \lambda_{T}^{2} \lambda_{T}^{*, 2}-9\left|\lambda_{T}\right|^{2} \operatorname{Tr}\left(Y_{d} Y_{d}^{\dagger}\right)-3\left|\lambda_{T}\right|^{2} \operatorname{Tr}\left(Y_{e} Y_{e}^{\dagger}\right) \\
& -\left|\lambda_{S}\right|^{2}\left(3 \operatorname{Tr}\left(Y_{d} Y_{d}^{\dagger}\right)+6 \lambda_{T} \lambda_{T}^{*}+\operatorname{Tr}\left(Y_{e} Y_{e}^{\dagger}\right)\right)+\frac{4}{5} g_{1}^{2} \operatorname{Tr}\left(Y_{u} Y_{u}^{\dagger}\right)+16 g_{3}^{2} \operatorname{Tr}\left(Y_{u} Y_{u}^{\dagger}\right) \\
& \left.-3 \operatorname{Tr}\left(Y_{d} Y_{u}^{\dagger} Y_{u} Y_{d}^{\dagger}\right)-9 \operatorname{Tr}\left(Y_{u} Y_{u}^{\dagger} Y_{u} Y_{u}^{\dagger}\right)\right) \\
& \beta_{Y_{d}}^{(1)}=3 Y_{d} Y_{d}^{\dagger} Y_{d}+Y_{d}\left(-3 g_{2}^{2}+3\left|\lambda_{T}\right|^{2}+3 \operatorname{Tr}\left(Y_{d} Y_{d}^{\dagger}\right)-\frac{16}{3} g_{3}^{2}-\frac{7}{15} g_{1}^{2}+\left|\lambda_{S}\right|^{2}\right. \\
& \left.+\operatorname{Tr}\left(Y_{e} Y_{e}^{\dagger}\right)\right)+Y_{d} Y_{u}^{\dagger} Y_{u} \\
& \beta_{Y_{d}}^{(2)}=+\frac{4}{5} g_{1}^{2} Y_{d} Y_{u}^{\dagger} Y_{u}-\left|\lambda_{S}\right|^{2} Y_{d} Y_{u}^{\dagger} Y_{u}-3\left|\lambda_{T}\right|^{2} Y_{d} Y_{u}^{\dagger} Y_{u}-4 Y_{d} Y_{d}^{\dagger} Y_{d} Y_{d}^{\dagger} Y_{d} \\
& -2 Y_{d} Y_{u}^{\dagger} Y_{u} Y_{d}^{\dagger} Y_{d}-2 Y_{d} Y_{u}^{\dagger} Y_{u} Y_{u}^{\dagger} Y_{u} \\
& +Y_{d} Y_{d}^{\dagger} Y_{d}\left(-3\left|\lambda_{S}\right|^{2}-3 \operatorname{Tr}\left(Y_{e} Y_{e}^{\dagger}\right)+6 g_{2}^{2}-9\left|\lambda_{T}\right|^{2}-9 \operatorname{Tr}\left(Y_{d} Y_{d}^{\dagger}\right)+\frac{4}{5} g_{1}^{2}\right) \\
& -3 Y_{d} Y_{u}^{\dagger} Y_{u} \operatorname{Tr}\left(Y_{u} Y_{u}^{\dagger}\right) \\
& +Y_{d}\left(\frac{287}{90} g_{1}^{4}+g_{1}^{2} g_{2}^{2}+\frac{27}{2} g_{2}^{4}+\frac{8}{9} g_{1}^{2} g_{3}^{2}+8 g_{2}^{2} g_{3}^{2}+\frac{128}{9} g_{3}^{4}+12 g_{2}^{2}\left|\lambda_{T}\right|^{2}-2 \lambda_{S}\left|\kappa_{S}\right|^{2} \lambda_{S}^{*}\right. \\
& -3 \lambda_{S}^{2} \lambda_{S}^{*, 2}-15 \lambda_{T}^{2} \lambda_{T}^{*, 2}-\frac{2}{5} g_{1}^{2} \operatorname{Tr}\left(Y_{d} Y_{d}^{\dagger}\right)+16 g_{3}^{2} \operatorname{Tr}\left(Y_{d} Y_{d}^{\dagger}\right)+\frac{6}{5} g_{1}^{2} \operatorname{Tr}\left(Y_{e} Y_{e}^{\dagger}\right) \\
& -9\left|\lambda_{T}\right|^{2} \operatorname{Tr}\left(Y_{u} Y_{u}^{\dagger}\right)-3\left|\lambda_{S}\right|^{2}\left(2 \lambda_{T} \lambda_{T}^{*}+\operatorname{Tr}\left(Y_{u} Y_{u}^{\dagger}\right)\right)-9 \operatorname{Tr}\left(Y_{d} Y_{d}^{\dagger} Y_{d} Y_{d}^{\dagger}\right) \\
& \left.-3 \operatorname{Tr}\left(Y_{d} Y_{u}^{\dagger} Y_{u} Y_{d}^{\dagger}\right)-3 \operatorname{Tr}\left(Y_{e} Y_{e}^{\dagger} Y_{e} Y_{e}^{\dagger}\right)\right) \\
& \beta_{Y_{e}}^{(1)}=3 Y_{e} Y_{e}^{\dagger} Y_{e}+Y_{e}\left(-3 g_{2}^{2}+3\left|\lambda_{T}\right|^{2}+3 \operatorname{Tr}\left(Y_{d} Y_{d}^{\dagger}\right)-\frac{9}{5} g_{1}^{2}+\left|\lambda_{S}\right|^{2}+\operatorname{Tr}\left(Y_{e} Y_{e}^{\dagger}\right)\right) \\
& \beta_{Y_{e}}^{(2)}=-4 Y_{e} Y_{e}^{\dagger} Y_{e} Y_{e}^{\dagger} Y_{e}+Y_{e} Y_{e}^{\dagger} Y_{e}\left(-3\left|\lambda_{S}\right|^{2}-3 \operatorname{Tr}\left(Y_{e} Y_{e}^{\dagger}\right)+6 g_{2}^{2}-9\left|\lambda_{T}\right|^{2}-9 \operatorname{Tr}\left(Y_{d} Y_{d}^{\dagger}\right)\right)
\end{aligned}
$$




$$
\begin{aligned}
& +Y_{e}\left(\frac{27}{2} g_{1}^{4}+\frac{9}{5} g_{1}^{2} g_{2}^{2}+\frac{27}{2} g_{2}^{4}+12 g_{2}^{2}\left|\lambda_{T}\right|^{2}-2 \lambda_{S}\left|\kappa_{S}\right|^{2} \lambda_{S}^{*}-3 \lambda_{S}^{2} \lambda_{S}^{*, 2}-15 \lambda_{T}^{2} \lambda_{T}^{*, 2}\right. \\
& -\frac{2}{5} g_{1}^{2} \operatorname{Tr}\left(Y_{d} Y_{d}^{\dagger}\right)+16 g_{3}^{2} \operatorname{Tr}\left(Y_{d} Y_{d}^{\dagger}\right)+\frac{6}{5} g_{1}^{2} \operatorname{Tr}\left(Y_{e} Y_{e}^{\dagger}\right)-9\left|\lambda_{T}\right|^{2} \operatorname{Tr}\left(Y_{u} Y_{u}^{\dagger}\right) \\
& -3\left|\lambda_{S}\right|^{2}\left(2 \lambda_{T} \lambda_{T}^{*}+\operatorname{Tr}\left(Y_{u} Y_{u}^{\dagger}\right)\right)-9 \operatorname{Tr}\left(Y_{d} Y_{d}^{\dagger} Y_{d} Y_{d}^{\dagger}\right)-3 \operatorname{Tr}\left(Y_{d} Y_{u}^{\dagger} Y_{u} Y_{d}^{\dagger}\right) \\
& \left.-3 \operatorname{Tr}\left(Y_{e} Y_{e}^{\dagger} Y_{e} Y_{e}^{\dagger}\right)\right) \\
& \beta_{\lambda_{T}}^{(1)}=2 \lambda_{T}\left|\lambda_{S}\right|^{2}+3 \lambda_{T} \operatorname{Tr}\left(Y_{d} Y_{d}^{\dagger}\right)+3 \lambda_{T} \operatorname{Tr}\left(Y_{u} Y_{u}^{\dagger}\right)-7 g_{2}^{2} \lambda_{T}+8 \lambda_{T}^{2} \lambda_{T}^{*}-\frac{3}{5} g_{1}^{2} \lambda_{T}+\lambda_{T} \operatorname{Tr}\left(Y_{e} Y_{e}^{\dagger}\right) \\
& \beta_{\lambda_{T}}^{(2)}=-\frac{1}{50} \lambda_{T}\left(-207 g_{1}^{4}-90 g_{1}^{2} g_{2}^{2}-2075 g_{2}^{4}-60 g_{1}^{2}\left|\lambda_{T}\right|^{2}-1100 g_{2}^{2}\left|\lambda_{T}\right|^{2}+200 \lambda_{S}\left|\kappa_{S}\right|^{2} \lambda_{S}^{*}\right. \\
& +300 \lambda_{S}^{2} \lambda_{S}^{*, 2}+2100 \lambda_{T}^{2} \lambda_{T}^{*, 2}+20 g_{1}^{2} \operatorname{Tr}\left(Y_{d} Y_{d}^{\dagger}\right)-800 g_{3}^{2} \operatorname{Tr}\left(Y_{d} Y_{d}^{\dagger}\right)+750\left|\lambda_{T}\right|^{2} \operatorname{Tr}\left(Y_{d} Y_{d}^{\dagger}\right) \\
& -60 g_{1}^{2} \operatorname{Tr}\left(Y_{e} Y_{e}^{\dagger}\right)+250\left|\lambda_{T}\right|^{2} \operatorname{Tr}\left(Y_{e} Y_{e}^{\dagger}\right)-40 g_{1}^{2} \operatorname{Tr}\left(Y_{u} Y_{u}^{\dagger}\right)-800 g_{3}^{2} \operatorname{Tr}\left(Y_{u} Y_{u}^{\dagger}\right) \\
& +750\left|\lambda_{T}\right|^{2} \operatorname{Tr}\left(Y_{u} Y_{u}^{\dagger}\right)+50\left|\lambda_{S}\right|^{2}\left(16 \lambda_{T} \lambda_{T}^{*}+3 \operatorname{Tr}\left(Y_{d} Y_{d}^{\dagger}\right)+3 \operatorname{Tr}\left(Y_{u} Y_{u}^{\dagger}\right)+\operatorname{Tr}\left(Y_{e} Y_{e}^{\dagger}\right)\right) \\
& \left.+450 \operatorname{Tr}\left(Y_{d} Y_{d}^{\dagger} Y_{d} Y_{d}^{\dagger}\right)+300 \operatorname{Tr}\left(Y_{d} Y_{u}^{\dagger} Y_{u} Y_{d}^{\dagger}\right)+150 \operatorname{Tr}\left(Y_{e} Y_{e}^{\dagger} Y_{e} Y_{e}^{\dagger}\right)+450 \operatorname{Tr}\left(Y_{u} Y_{u}^{\dagger} Y_{u} Y_{u}^{\dagger}\right)\right) \\
& \beta_{\lambda_{S}}^{(1)}=-\frac{3}{5} g_{1}^{2} \lambda_{S}-3 g_{2}^{2} \lambda_{S}+2 \lambda_{S}\left|\kappa_{S}\right|^{2}+6 \lambda_{S}\left|\lambda_{T}\right|^{2}+4 \lambda_{S}^{2} \lambda_{S}^{*}+3 \lambda_{S} \operatorname{Tr}\left(Y_{d} Y_{d}^{\dagger}\right)+\lambda_{S} \operatorname{Tr}\left(Y_{e} Y_{e}^{\dagger}\right) \\
& +3 \lambda_{S} \operatorname{Tr}\left(Y_{u} Y_{u}^{\dagger}\right) \\
& \beta_{\lambda_{S}}^{(2)}=-\frac{1}{50} \lambda_{S}\left(-207 g_{1}^{4}-90 g_{1}^{2} g_{2}^{2}-675 g_{2}^{4}-1200 g_{2}^{2}\left|\lambda_{T}\right|^{2}+400 \kappa_{S}^{2} \kappa_{S}^{* 2}+600 \lambda_{S}\left|\kappa_{S}\right|^{2} \lambda_{S}^{*}\right. \\
& +500 \lambda_{S}^{2} \lambda_{S}^{*, 2}+1500 \lambda_{T}^{2} \lambda_{T}^{*, 2}+20 g_{1}^{2} \operatorname{Tr}\left(Y_{d} Y_{d}^{\dagger}\right)-800 g_{3}^{2} \operatorname{Tr}\left(Y_{d} Y_{d}^{\dagger}\right) \\
& +450\left|\lambda_{T}\right|^{2} \operatorname{Tr}\left(Y_{d} Y_{d}^{\dagger}\right)-60 g_{1}^{2} \operatorname{Tr}\left(Y_{e} Y_{e}^{\dagger}\right)+150\left|\lambda_{T}\right|^{2} \operatorname{Tr}\left(Y_{e} Y_{e}^{\dagger}\right) \\
& -30\left|\lambda_{S}\right|^{2}\left(10 g_{2}^{2}-15 \operatorname{Tr}\left(Y_{d} Y_{d}^{\dagger}\right)-15 \operatorname{Tr}\left(Y_{u} Y_{u}^{\dagger}\right)+2 g_{1}^{2}-40 \lambda_{T} \lambda_{T}^{*}-5 \operatorname{Tr}\left(Y_{e} Y_{e}^{\dagger}\right)\right) \\
& -40 g_{1}^{2} \operatorname{Tr}\left(Y_{u} Y_{u}^{\dagger}\right)-800 g_{3}^{2} \operatorname{Tr}\left(Y_{u} Y_{u}^{\dagger}\right)+450\left|\lambda_{T}\right|^{2} \operatorname{Tr}\left(Y_{u} Y_{u}^{\dagger}\right)+450 \operatorname{Tr}\left(Y_{d} Y_{d}^{\dagger} Y_{d} Y_{d}^{\dagger}\right) \\
& \left.+300 \operatorname{Tr}\left(Y_{d} Y_{u}^{\dagger} Y_{u} Y_{d}^{\dagger}\right)+150 \operatorname{Tr}\left(Y_{e} Y_{e}^{\dagger} Y_{e} Y_{e}^{\dagger}\right)+450 \operatorname{Tr}\left(Y_{u} Y_{u}^{\dagger} Y_{u} Y_{u}^{\dagger}\right)\right) \\
& \beta_{\kappa_{S}}^{(1)}=6 \kappa_{S}\left(\left|\kappa_{S}\right|^{2}+\left|\lambda_{S}\right|^{2}\right) \\
& \beta_{\kappa_{S}}^{(2)}=-\frac{6}{5} \kappa_{S}\left(20 \kappa_{S}^{2} \kappa_{S}^{*, 2}+20 \lambda_{S}\left|\kappa_{S}\right|^{2} \lambda_{S}^{*}\right. \\
& +\left|\lambda_{S}\right|^{2}\left(10 \lambda_{S} \lambda_{S}^{*}-15 g_{2}^{2}+15 \operatorname{Tr}\left(Y_{d} Y_{d}^{\dagger}\right)+15 \operatorname{Tr}\left(Y_{u} Y_{u}^{\dagger}\right)+30 \lambda_{T} \lambda_{T}^{*}-3 g_{1}^{2}\right. \\
& \left.\left.+5 \operatorname{Tr}\left(Y_{e} Y_{e}^{\dagger}\right)\right)\right)
\end{aligned}
$$

\section{B.5 Bilinear superpotential parameters}

$$
\begin{aligned}
& \beta_{\mu}^{(1)}=2 \mu\left|\lambda_{S}\right|^{2}-3 g_{2}^{2} \mu+3 \mu \operatorname{Tr}\left(Y_{d} Y_{d}^{\dagger}\right)+3 \mu \operatorname{Tr}\left(Y_{u} Y_{u}^{\dagger}\right)+6 \mu\left|\lambda_{T}\right|^{2}-\frac{3}{5} g_{1}^{2} \mu+\mu \operatorname{Tr}\left(Y_{e} Y_{e}^{\dagger}\right) \\
& \beta_{\mu}^{(2)}=+\frac{207}{50} g_{1}^{4} \mu+\frac{9}{5} g_{1}^{2} g_{2}^{2} \mu+\frac{27}{2} g_{2}^{4} \mu+24 g_{2}^{2} \mu\left|\lambda_{T}\right|^{2}-4 \lambda_{S} \mu\left|\kappa_{S}\right|^{2} \lambda_{S}^{*}-6 \lambda_{S}^{2} \mu \lambda_{S}^{* 2}
\end{aligned}
$$




$$
\begin{aligned}
& -30 \lambda_{T}^{2} \mu \lambda_{T}^{*, 2}-\frac{2}{5} g_{1}^{2} \mu \operatorname{Tr}\left(Y_{d} Y_{d}^{\dagger}\right)+16 g_{3}^{2} \mu \operatorname{Tr}\left(Y_{d} Y_{d}^{\dagger}\right)-9 \mu\left|\lambda_{T}\right|^{2} \operatorname{Tr}\left(Y_{d} Y_{d}^{\dagger}\right) \\
& +\frac{6}{5} g_{1}^{2} \mu \operatorname{Tr}\left(Y_{e} Y_{e}^{\dagger}\right)-3 \mu\left|\lambda_{T}\right|^{2} \operatorname{Tr}\left(Y_{e} Y_{e}^{\dagger}\right)+\frac{4}{5} g_{1}^{2} \mu \operatorname{Tr}\left(Y_{u} Y_{u}^{\dagger}\right)+16 g_{3}^{2} \mu \operatorname{Tr}\left(Y_{u} Y_{u}^{\dagger}\right) \\
& -9 \mu\left|\lambda_{T}\right|^{2} \operatorname{Tr}\left(Y_{u} Y_{u}^{\dagger}\right)-\mu\left|\lambda_{S}\right|^{2}\left(12 \lambda_{T} \lambda_{T}^{*}+3 \operatorname{Tr}\left(Y_{d} Y_{d}^{\dagger}\right)+3 \operatorname{Tr}\left(Y_{u} Y_{u}^{\dagger}\right)+\operatorname{Tr}\left(Y_{e} Y_{e}^{\dagger}\right)\right) \\
& -9 \mu \operatorname{Tr}\left(Y_{d} Y_{d}^{\dagger} Y_{d} Y_{d}^{\dagger}\right)-6 \mu \operatorname{Tr}\left(Y_{d} Y_{u}^{\dagger} Y_{u} Y_{d}^{\dagger}\right)-3 \mu \operatorname{Tr}\left(Y_{e} Y_{e}^{\dagger} Y_{e} Y_{e}^{\dagger}\right)-9 \mu \operatorname{Tr}\left(Y_{u} Y_{u}^{\dagger} Y_{u} Y_{u}^{\dagger}\right) \\
\beta_{M_{T}}^{(1)}= & 4 M_{T}\left|\lambda_{T}\right|^{2}-8 g_{2}^{2} M_{T} \\
\beta_{M_{T}}^{(2)}= & \frac{4}{5} M_{T}\left(70 g_{2}^{4}-30 \lambda_{T}^{2} \lambda_{T}^{*, 2}\right. \\
& \left.+\left|\lambda_{T}\right|^{2}\left(-10 \lambda_{S} \lambda_{S}^{*}-15 \operatorname{Tr}\left(Y_{d} Y_{d}^{\dagger}\right)-15 \operatorname{Tr}\left(Y_{u} Y_{u}^{\dagger}\right)+3 g_{1}^{2}-5 g_{2}^{2}-5 \operatorname{Tr}\left(Y_{e} Y_{e}^{\dagger}\right)\right)\right) \\
\beta_{M_{O}}^{(1)}= & -12 g_{3}^{2} M_{O} \\
\beta_{M_{O}}^{(2)}= & 72 g_{3}^{4} M_{O} \\
\beta_{M_{S}}^{(1)}= & 4 M_{S}\left(\left|\kappa_{S}\right|^{2}+\left|\lambda_{S}\right|^{2}\right) \\
\beta_{M_{S}}^{(2)}= & -\frac{4}{5} M_{S}\left(20 \kappa_{S}^{2} \kappa_{S}^{* 2}+20 \lambda_{S}\left|\kappa_{S}\right|^{2} \lambda_{S}^{*}\right. \\
& +\left|\lambda_{S}\right|^{2}\left(10 \lambda_{S} \lambda_{S}^{*}-15 g_{2}^{2}+15 \operatorname{Tr}\left(Y_{d} Y_{d}^{\dagger}\right)+15 \operatorname{Tr}\left(Y_{u} Y_{u}^{\dagger}\right)+30 \lambda_{T} \lambda_{T}^{*}-3 g_{1}^{2}\right. \\
& \left.\left.+5 \operatorname{Tr}\left(Y_{e} Y_{e}^{\dagger}\right)\right)\right)
\end{aligned}
$$

\section{B.6 Linear superpotential parameters}

$$
\begin{aligned}
\beta_{L_{S}}^{(1)}= & 2 L_{S}\left(\left|\kappa_{S}\right|^{2}+\left|\lambda_{S}\right|^{2}\right) \\
\beta_{L_{S}}^{(2)}= & -\frac{2}{5} L_{S}\left(20 \kappa_{S}^{2} \kappa_{S}^{*, 2}+20 \lambda_{S}\left|\kappa_{S}\right|^{2} \lambda_{S}^{*}\right. \\
& +\left|\lambda_{S}\right|^{2}\left(10 \lambda_{S} \lambda_{S}^{*}-15 g_{2}^{2}+15 \operatorname{Tr}\left(Y_{d} Y_{d}^{\dagger}\right)+15 \operatorname{Tr}\left(Y_{u} Y_{u}^{\dagger}\right)+30 \lambda_{T} \lambda_{T}^{*}-3 g_{1}^{2}\right. \\
& \left.\left.+5 \operatorname{Tr}\left(Y_{e} Y_{e}^{\dagger}\right)\right)\right)
\end{aligned}
$$

\section{B.7 Trilinear soft-breaking parameters}

$$
\begin{aligned}
\beta_{A_{u}}^{(1)}= & +2 Y_{u} Y_{d}^{\dagger} A_{d}+4 Y_{u} Y_{u}^{\dagger} A_{u}+A_{u} Y_{d}^{\dagger} Y_{d}+5 A_{u} Y_{u}^{\dagger} Y_{u}-\frac{13}{15} g_{1}^{2} A_{u}-3 g_{2}^{2} A_{u}-\frac{16}{3} g_{3}^{2} A_{u} \\
& +\left|\lambda_{S}\right|^{2} A_{u}+3\left|\lambda_{T}\right|^{2} A_{u}+3 A_{u} \operatorname{Tr}\left(Y_{u} Y_{u}^{\dagger}\right) \\
& +Y_{u}\left(2 \lambda_{S}^{*} A_{S}+6 g_{2}^{2} M_{2}+6 \lambda_{T}^{*} A_{T}+6 \operatorname{Tr}\left(Y_{u}^{\dagger} A_{u}\right)+\frac{26}{15} g_{1}^{2} M_{1}+\frac{32}{3} g_{3}^{2} M_{3}\right) \\
\beta_{A_{u}}^{(2)}= & +\frac{4}{5} g_{1}^{2} Y_{u} Y_{d}^{\dagger} A_{d}-2\left|\lambda_{S}\right|^{2} Y_{u} Y_{d}^{\dagger} A_{d}-6\left|\lambda_{T}\right|^{2} Y_{u} Y_{d}^{\dagger} A_{d}-\frac{4}{5} g_{1}^{2} M_{1} Y_{u} Y_{u}^{\dagger} Y_{u} \\
& -12 g_{2}^{2} M_{2} Y_{u} Y_{u}^{\dagger} Y_{u}+\frac{6}{5} g_{1}^{2} Y_{u} Y_{u}^{\dagger} A_{u}+6 g_{2}^{2} Y_{u} Y_{u}^{\dagger} A_{u}-4\left|\lambda_{S}\right|^{2} Y_{u} Y_{u}^{\dagger} A_{u} \\
& -12\left|\lambda_{T}\right|^{2} Y_{u} Y_{u}^{\dagger} A_{u}+\frac{2}{5} g_{1}^{2} A_{u} Y_{d}^{\dagger} Y_{d}-\left|\lambda_{S}\right|^{2} A_{u} Y_{d}^{\dagger} Y_{d}-3\left|\lambda_{T}\right|^{2} A_{u} Y_{d}^{\dagger} Y_{d} \\
& +12 g_{2}^{2} A_{u} Y_{u}^{\dagger} Y_{u}-5\left|\lambda_{S}\right|^{2} A_{u} Y_{u}^{\dagger} Y_{u}-15\left|\lambda_{T}\right|^{2} A_{u} Y_{u}^{\dagger} Y_{u}-4 Y_{u} Y_{d}^{\dagger} Y_{d} Y_{d}^{\dagger} A_{d}
\end{aligned}
$$




$$
\begin{aligned}
& -2 Y_{u} Y_{d}^{\dagger} Y_{d} Y_{u}^{\dagger} A_{u}-4 Y_{u} Y_{d}^{\dagger} A_{d} Y_{d}^{\dagger} Y_{d}-4 Y_{u} Y_{d}^{\dagger} A_{d} Y_{u}^{\dagger} Y_{u}-6 Y_{u} Y_{u}^{\dagger} Y_{u} Y_{u}^{\dagger} A_{u} \\
& -8 Y_{u} Y_{u}^{\dagger} A_{u} Y_{u}^{\dagger} Y_{u}-2 A_{u} Y_{d}^{\dagger} Y_{d} Y_{d}^{\dagger} Y_{d}-4 A_{u} Y_{d}^{\dagger} Y_{d} Y_{u}^{\dagger} Y_{u}-6 A_{u} Y_{u}^{\dagger} Y_{u} Y_{u}^{\dagger} Y_{u}+\frac{2743}{450} g_{1}^{4} A_{u} \\
& +g_{1}^{2} g_{2}^{2} A_{u}+\frac{27}{2} g_{2}^{4} A_{u}+\frac{136}{45} g_{1}^{2} g_{3}^{2} A_{u}+8 g_{2}^{2} g_{3}^{2} A_{u}+\frac{128}{9} g_{3}^{4} A_{u}+12 g_{2}^{2}\left|\lambda_{T}\right|^{2} A_{u} \\
& -2 \lambda_{S}\left|\kappa_{S}\right|^{2} \lambda_{S}^{*} A_{u}-3 \lambda_{S}^{2} \lambda_{S}^{*, 2} A_{u}-6 \lambda_{T}\left|\lambda_{S}\right|^{2} \lambda_{T}^{*} A_{u}-15 \lambda_{T}^{2} \lambda_{T}^{*, 2} A_{u}-6 \lambda_{S}^{*} Y_{u} Y_{u}^{\dagger} Y_{u} A_{S} \\
& -18 \lambda_{T}^{*} Y_{u} Y_{u}^{\dagger} Y_{u} A_{T}-6 Y_{u} Y_{d}^{\dagger} A_{d} \operatorname{Tr}\left(Y_{d} Y_{d}^{\dagger}\right)-3 A_{u} Y_{d}^{\dagger} Y_{d} \operatorname{Tr}\left(Y_{d} Y_{d}^{\dagger}\right) \\
& -3\left|\lambda_{S}\right|^{2} A_{u} \operatorname{Tr}\left(Y_{d} Y_{d}^{\dagger}\right)-9\left|\lambda_{T}\right|^{2} A_{u} \operatorname{Tr}\left(Y_{d} Y_{d}^{\dagger}\right)-2 Y_{u} Y_{d}^{\dagger} A_{d} \operatorname{Tr}\left(Y_{e} Y_{e}^{\dagger}\right) \\
& -A_{u} Y_{d}^{\dagger} Y_{d} \operatorname{Tr}\left(Y_{e} Y_{e}^{\dagger}\right)-\left|\lambda_{S}\right|^{2} A_{u} \operatorname{Tr}\left(Y_{e} Y_{e}^{\dagger}\right)-3\left|\lambda_{T}\right|^{2} A_{u} \operatorname{Tr}\left(Y_{e} Y_{e}^{\dagger}\right) \\
& -12 Y_{u} Y_{u}^{\dagger} A_{u} \operatorname{Tr}\left(Y_{u} Y_{u}^{\dagger}\right)-15 A_{u} Y_{u}^{\dagger} Y_{u} \operatorname{Tr}\left(Y_{u} Y_{u}^{\dagger}\right)+\frac{4}{5} g_{1}^{2} A_{u} \operatorname{Tr}\left(Y_{u} Y_{u}^{\dagger}\right) \\
& +16 g_{3}^{2} A_{u} \operatorname{Tr}\left(Y_{u} Y_{u}^{\dagger}\right)-\frac{2}{5} Y_{u} Y_{d}^{\dagger} Y_{d}\left(15 \lambda_{T}^{*} A_{T}+15 \operatorname{Tr}\left(Y_{d}^{\dagger} A_{d}\right)+2 g_{1}^{2} M_{1}+5 \lambda_{S}^{*} A_{S}\right. \\
& \left.+5 \operatorname{Tr}\left(Y_{e}^{\dagger} A_{e}\right)\right)-18 Y_{u} Y_{u}^{\dagger} Y_{u} \operatorname{Tr}\left(Y_{u}^{\dagger} A_{u}\right)-3 A_{u} \operatorname{Tr}\left(Y_{d} Y_{u}^{\dagger} Y_{u} Y_{d}^{\dagger}\right)-9 A_{u} \operatorname{Tr}\left(Y_{u} Y_{u}^{\dagger} Y_{u} Y_{u}^{\dagger}\right) \\
& -\frac{2}{225} Y_{u}\left(2743 g_{1}^{4} M_{1}+225 g_{1}^{2} g_{2}^{2} M_{1}+680 g_{1}^{2} g_{3}^{2} M_{1}\right. \\
& +680 g_{1}^{2} g_{3}^{2} M_{3}+1800 g_{2}^{2} g_{3}^{2} M_{3}+6400 g_{3}^{4} M_{3} \\
& +225 g_{1}^{2} g_{2}^{2} M_{2}+6075 g_{2}^{4} M_{2}+1800 g_{2}^{2} g_{3}^{2} M_{2}+1350 \lambda_{S} \lambda_{S}^{*, 2} A_{S}+450 \kappa_{S}^{*} \lambda_{S}^{*}\left(\kappa_{S} A_{S}+\lambda_{S} A_{\kappa}\right) \\
& +6750 \lambda_{T} \lambda_{T}^{*, 2} A_{T}+675 \lambda_{S}^{*} A_{S} \operatorname{Tr}\left(Y_{d} Y_{d}^{\dagger}\right)+225 \lambda_{S}^{*} A_{S} \operatorname{Tr}\left(Y_{e} Y_{e}^{\dagger}\right)+180 g_{1}^{2} M_{1} \operatorname{Tr}\left(Y_{u} Y_{u}^{\dagger}\right) \\
& +3600 g_{3}^{2} M_{3} \operatorname{Tr}\left(Y_{u} Y_{u}^{\dagger}\right)+675\left|\lambda_{S}\right|^{2} \operatorname{Tr}\left(Y_{d}^{\dagger} A_{d}\right)+225\left|\lambda_{S}\right|^{2} \operatorname{Tr}\left(Y_{e}^{\dagger} A_{e}\right) \\
& +675 \lambda_{T}^{*}\left(2 \lambda_{S}^{*}\left(\lambda_{S} A_{T}+\lambda_{T} A_{S}\right)\right. \\
& \left.+A_{T}\left(3 \operatorname{Tr}\left(Y_{d} Y_{d}^{\dagger}\right)-4 g_{2}^{2}+\operatorname{Tr}\left(Y_{e} Y_{e}^{\dagger}\right)\right)+\lambda_{T}\left(3 \operatorname{Tr}\left(Y_{d}^{\dagger} A_{d}\right)+4 g_{2}^{2} M_{2}+\operatorname{Tr}\left(Y_{e}^{\dagger} A_{e}\right)\right)\right) \\
& -180 g_{1}^{2} \operatorname{Tr}\left(Y_{u}^{\dagger} A_{u}\right)-3600 g_{3}^{2} \operatorname{Tr}\left(Y_{u}^{\dagger} A_{u}\right)+675 \operatorname{Tr}\left(Y_{d} Y_{u}^{\dagger} A_{u} Y_{d}^{\dagger}\right)+675 \operatorname{Tr}\left(Y_{u} Y_{d}^{\dagger} A_{d} Y_{u}^{\dagger}\right) \\
& \left.+4050 \operatorname{Tr}\left(Y_{u} Y_{u}^{\dagger} A_{u} Y_{u}^{\dagger}\right)\right) \\
& \beta_{A_{d}}^{(1)}=+4 Y_{d} Y_{d}^{\dagger} A_{d}+2 Y_{d} Y_{u}^{\dagger} A_{u}+5 A_{d} Y_{d}^{\dagger} Y_{d}+A_{d} Y_{u}^{\dagger} Y_{u}-\frac{7}{15} g_{1}^{2} A_{d}-3 g_{2}^{2} A_{d}-\frac{16}{3} g_{3}^{2} A_{d} \\
& +\left|\lambda_{S}\right|^{2} A_{d}+3\left|\lambda_{T}\right|^{2} A_{d}+3 A_{d} \operatorname{Tr}\left(Y_{d} Y_{d}^{\dagger}\right)+A_{d} \operatorname{Tr}\left(Y_{e} Y_{e}^{\dagger}\right) \\
& +Y_{d}\left(2 \lambda_{S}^{*} A_{S}+2 \operatorname{Tr}\left(Y_{e}^{\dagger} A_{e}\right)+6 g_{2}^{2} M_{2}+6 \lambda_{T}^{*} A_{T}+6 \operatorname{Tr}\left(Y_{d}^{\dagger} A_{d}\right)+\frac{14}{15} g_{1}^{2} M_{1}+\frac{32}{3} g_{3}^{2} M_{3}\right) \\
& \beta_{A_{d}}^{(2)}=+\frac{6}{5} g_{1}^{2} Y_{d} Y_{d}^{\dagger} A_{d}+6 g_{2}^{2} Y_{d} Y_{d}^{\dagger} A_{d}-4\left|\lambda_{S}\right|^{2} Y_{d} Y_{d}^{\dagger} A_{d}-12\left|\lambda_{T}\right|^{2} Y_{d} Y_{d}^{\dagger} A_{d} \\
& -\frac{8}{5} g_{1}^{2} M_{1} Y_{d} Y_{u}^{\dagger} Y_{u}+\frac{8}{5} g_{1}^{2} Y_{d} Y_{u}^{\dagger} A_{u}-2\left|\lambda_{S}\right|^{2} Y_{d} Y_{u}^{\dagger} A_{u}-6\left|\lambda_{T}\right|^{2} Y_{d} Y_{u}^{\dagger} A_{u} \\
& +\frac{6}{5} g_{1}^{2} A_{d} Y_{d}^{\dagger} Y_{d}+12 g_{2}^{2} A_{d} Y_{d}^{\dagger} Y_{d}-5\left|\lambda_{S}\right|^{2} A_{d} Y_{d}^{\dagger} Y_{d}-15\left|\lambda_{T}\right|^{2} A_{d} Y_{d}^{\dagger} Y_{d} \\
& +\frac{4}{5} g_{1}^{2} A_{d} Y_{u}^{\dagger} Y_{u}-\left|\lambda_{S}\right|^{2} A_{d} Y_{u}^{\dagger} Y_{u}-3\left|\lambda_{T}\right|^{2} A_{d} Y_{u}^{\dagger} Y_{u}-6 Y_{d} Y_{d}^{\dagger} Y_{d} Y_{d}^{\dagger} A_{d}
\end{aligned}
$$




$$
\begin{aligned}
& -8 Y_{d} Y_{d}^{\dagger} A_{d} Y_{d}^{\dagger} Y_{d}-2 Y_{d} Y_{u}^{\dagger} Y_{u} Y_{d}^{\dagger} A_{d}-4 Y_{d} Y_{u}^{\dagger} Y_{u} Y_{u}^{\dagger} A_{u}-4 Y_{d} Y_{u}^{\dagger} A_{u} Y_{d}^{\dagger} Y_{d} \\
& -4 Y_{d} Y_{u}^{\dagger} A_{u} Y_{u}^{\dagger} Y_{u}-6 A_{d} Y_{d}^{\dagger} Y_{d} Y_{d}^{\dagger} Y_{d}-4 A_{d} Y_{u}^{\dagger} Y_{u} Y_{d}^{\dagger} Y_{d}-2 A_{d} Y_{u}^{\dagger} Y_{u} Y_{u}^{\dagger} Y_{u} \\
& +\frac{287}{90} g_{1}^{4} A_{d}+g_{1}^{2} g_{2}^{2} A_{d}+\frac{27}{2} g_{2}^{4} A_{d}+\frac{8}{9} g_{1}^{2} g_{3}^{2} A_{d}+8 g_{2}^{2} g_{3}^{2} A_{d}+\frac{128}{9} g_{3}^{4} A_{d}+12 g_{2}^{2}\left|\lambda_{T}\right|^{2} A_{d} \\
& -2 \lambda_{S}\left|\kappa_{S}\right|^{2} \lambda_{S}^{*} A_{d}-3 \lambda_{S}^{2} \lambda_{S}^{*, 2} A_{d}-6 \lambda_{T}\left|\lambda_{S}\right|^{2} \lambda_{T}^{*} A_{d}-15 \lambda_{T}^{2} \lambda_{T}^{*, 2} A_{d} \\
& -2 \lambda_{S}^{*} Y_{d} Y_{u}^{\dagger} Y_{u} A_{S}-6 \lambda_{T}^{*} Y_{d} Y_{u}^{\dagger} Y_{u} A_{T}-12 Y_{d} Y_{d}^{\dagger} A_{d} \operatorname{Tr}\left(Y_{d} Y_{d}^{\dagger}\right) \\
& -15 A_{d} Y_{d}^{\dagger} Y_{d} \operatorname{Tr}\left(Y_{d} Y_{d}^{\dagger}\right)-\frac{2}{5} g_{1}^{2} A_{d} \operatorname{Tr}\left(Y_{d} Y_{d}^{\dagger}\right)+16 g_{3}^{2} A_{d} \operatorname{Tr}\left(Y_{d} Y_{d}^{\dagger}\right) \\
& -4 Y_{d} Y_{d}^{\dagger} A_{d} \operatorname{Tr}\left(Y_{e} Y_{e}^{\dagger}\right)-5 A_{d} Y_{d}^{\dagger} Y_{d} \operatorname{Tr}\left(Y_{e} Y_{e}^{\dagger}\right)+\frac{6}{5} g_{1}^{2} A_{d} \operatorname{Tr}\left(Y_{e} Y_{e}^{\dagger}\right) \\
& -6 Y_{d} Y_{u}^{\dagger} A_{u} \operatorname{Tr}\left(Y_{u} Y_{u}^{\dagger}\right)-3 A_{d} Y_{u}^{\dagger} Y_{u} \operatorname{Tr}\left(Y_{u} Y_{u}^{\dagger}\right)-3\left|\lambda_{S}\right|^{2} A_{d} \operatorname{Tr}\left(Y_{u} Y_{u}^{\dagger}\right) \\
& -9\left|\lambda_{T}\right|^{2} A_{d} \operatorname{Tr}\left(Y_{u} Y_{u}^{\dagger}\right) \\
& -\frac{2}{5} Y_{d} Y_{d}^{\dagger} Y_{d}\left(15 \lambda_{S}^{*} A_{S}+15 \operatorname{Tr}\left(Y_{e}^{\dagger} A_{e}\right)+30 g_{2}^{2} M_{2}+45 \lambda_{T}^{*} A_{T}+45 \operatorname{Tr}\left(Y_{d}^{\dagger} A_{d}\right)+4 g_{1}^{2} M_{1}\right) \\
& -6 Y_{d} Y_{u}^{\dagger} Y_{u} \operatorname{Tr}\left(Y_{u}^{\dagger} A_{u}\right)-9 A_{d} \operatorname{Tr}\left(Y_{d} Y_{d}^{\dagger} Y_{d} Y_{d}^{\dagger}\right)-3 A_{d} \operatorname{Tr}\left(Y_{d} Y_{u}^{\dagger} Y_{u} Y_{d}^{\dagger}\right) \\
& -3 A_{d} \operatorname{Tr}\left(Y_{e} Y_{e}^{\dagger} Y_{e} Y_{e}^{\dagger}\right) \\
& -\frac{2}{45} Y_{d}\left(287 g_{1}^{4} M_{1}+45 g_{1}^{2} g_{2}^{2} M_{1}+40 g_{1}^{2} g_{3}^{2} M_{1}+40 g_{1}^{2} g_{3}^{2} M_{3}+360 g_{2}^{2} g_{3}^{2} M_{3}+1280 g_{3}^{4} M_{3}\right. \\
& +45 g_{1}^{2} g_{2}^{2} M_{2}+1215 g_{2}^{4} M_{2}+360 g_{2}^{2} g_{3}^{2} M_{2}+270 \lambda_{S} \lambda_{S}^{* 2} A_{S}+90 \kappa_{S}^{*} \lambda_{S}^{*}\left(\kappa_{S} A_{S}+\lambda_{S} A_{\kappa}\right) \\
& +1350 \lambda_{T} \lambda_{T}^{*, 2} A_{T}-18 g_{1}^{2} M_{1} \operatorname{Tr}\left(Y_{d} Y_{d}^{\dagger}\right)+720 g_{3}^{2} M_{3} \operatorname{Tr}\left(Y_{d} Y_{d}^{\dagger}\right)+54 g_{1}^{2} M_{1} \operatorname{Tr}\left(Y_{e} Y_{e}^{\dagger}\right) \\
& +135 \lambda_{S}^{*} A_{S} \operatorname{Tr}\left(Y_{u} Y_{u}^{\dagger}\right)+18 g_{1}^{2} \operatorname{Tr}\left(Y_{d}^{\dagger} A_{d}\right)-720 g_{3}^{2} \operatorname{Tr}\left(Y_{d}^{\dagger} A_{d}\right)-54 g_{1}^{2} \operatorname{Tr}\left(Y_{e}^{\dagger} A_{e}\right) \\
& +135\left|\lambda_{S}\right|^{2} \operatorname{Tr}\left(Y_{u}^{\dagger} A_{u}\right) \\
& +135 \lambda_{T}^{*}\left(2 \lambda_{S}^{*}\left(\lambda_{S} A_{T}+\lambda_{T} A_{S}\right)+3 A_{T} \operatorname{Tr}\left(Y_{u} Y_{u}^{\dagger}\right)+3 \lambda_{T} \operatorname{Tr}\left(Y_{u}^{\dagger} A_{u}\right)\right. \\
& \left.-4 g_{2}^{2} A_{T}+4 g_{2}^{2} M_{2} \lambda_{T}\right) \\
& +810 \operatorname{Tr}\left(Y_{d} Y_{d}^{\dagger} A_{d} Y_{d}^{\dagger}\right)+135 \operatorname{Tr}\left(Y_{d} Y_{u}^{\dagger} A_{u} Y_{d}^{\dagger}\right) \\
& \left.+270 \operatorname{Tr}\left(Y_{e} Y_{e}^{\dagger} A_{e} Y_{e}^{\dagger}\right)+135 \operatorname{Tr}\left(Y_{u} Y_{d}^{\dagger} A_{d} Y_{u}^{\dagger}\right)\right) \\
& \beta_{A_{e}}^{(1)}=+4 Y_{e} Y_{e}^{\dagger} A_{e}+5 A_{e} Y_{e}^{\dagger} Y_{e}-\frac{9}{5} g_{1}^{2} A_{e}-3 g_{2}^{2} A_{e}+\left|\lambda_{S}\right|^{2} A_{e}+3\left|\lambda_{T}\right|^{2} A_{e}+3 A_{e} \operatorname{Tr}\left(Y_{d} Y_{d}^{\dagger}\right) \\
& +A_{e} \operatorname{Tr}\left(Y_{e} Y_{e}^{\dagger}\right)+Y_{e}\left(2 \lambda_{S}^{*} A_{S}+2 \operatorname{Tr}\left(Y_{e}^{\dagger} A_{e}\right)+6 g_{2}^{2} M_{2}+6 \lambda_{T}^{*} A_{T}\right. \\
& \left.+6 \operatorname{Tr}\left(Y_{d}^{\dagger} A_{d}\right)+\frac{18}{5} g_{1}^{2} M_{1}\right) \\
& \beta_{A_{e}}^{(2)}=+\frac{6}{5} g_{1}^{2} Y_{e} Y_{e}^{\dagger} A_{e}+6 g_{2}^{2} Y_{e} Y_{e}^{\dagger} A_{e}-4\left|\lambda_{S}\right|^{2} Y_{e} Y_{e}^{\dagger} A_{e}-12\left|\lambda_{T}\right|^{2} Y_{e} Y_{e}^{\dagger} A_{e} \\
& -\frac{6}{5} g_{1}^{2} A_{e} Y_{e}^{\dagger} Y_{e}+12 g_{2}^{2} A_{e} Y_{e}^{\dagger} Y_{e}-5\left|\lambda_{S}\right|^{2} A_{e} Y_{e}^{\dagger} Y_{e}-15\left|\lambda_{T}\right|^{2} A_{e} Y_{e}^{\dagger} Y_{e}
\end{aligned}
$$




$$
\begin{aligned}
& -6 Y_{e} Y_{e}^{\dagger} Y_{e} Y_{e}^{\dagger} A_{e}-8 Y_{e} Y_{e}^{\dagger} A_{e} Y_{e}^{\dagger} Y_{e}-6 A_{e} Y_{e}^{\dagger} Y_{e} Y_{e}^{\dagger} Y_{e}+\frac{27}{2} g_{1}^{4} A_{e}+\frac{9}{5} g_{1}^{2} g_{2}^{2} A_{e}+\frac{27}{2} g_{2}^{4} A_{e} \\
& +12 g_{2}^{2}\left|\lambda_{T}\right|^{2} A_{e}-2 \lambda_{S}\left|\kappa_{S}\right|^{2} \lambda_{S}^{*} A_{e}-3 \lambda_{S}^{2} \lambda_{S}^{*, 2} A_{e}-6 \lambda_{T}\left|\lambda_{S}\right|^{2} \lambda_{T}^{*} A_{e}-15 \lambda_{T}^{2} \lambda_{T}^{*, 2} A_{e} \\
& -12 Y_{e} Y_{e}^{\dagger} A_{e} \operatorname{Tr}\left(Y_{d} Y_{d}^{\dagger}\right)-15 A_{e} Y_{e}^{\dagger} Y_{e} \operatorname{Tr}\left(Y_{d} Y_{d}^{\dagger}\right)-\frac{2}{5} g_{1}^{2} A_{e} \operatorname{Tr}\left(Y_{d} Y_{d}^{\dagger}\right) \\
& +16 g_{3}^{2} A_{e} \operatorname{Tr}\left(Y_{d} Y_{d}^{\dagger}\right)-4 Y_{e} Y_{e}^{\dagger} A_{e} \operatorname{Tr}\left(Y_{e} Y_{e}^{\dagger}\right)-5 A_{e} Y_{e}^{\dagger} Y_{e} \operatorname{Tr}\left(Y_{e} Y_{e}^{\dagger}\right) \\
& +\frac{6}{5} g_{1}^{2} A_{e} \operatorname{Tr}\left(Y_{e} Y_{e}^{\dagger}\right)-3\left|\lambda_{S}\right|^{2} A_{e} \operatorname{Tr}\left(Y_{u} Y_{u}^{\dagger}\right)-9\left|\lambda_{T}\right|^{2} A_{e} \operatorname{Tr}\left(Y_{u} Y_{u}^{\dagger}\right) \\
& -6 Y_{e} Y_{e}^{\dagger} Y_{e}\left(2 g_{2}^{2} M_{2}+3 \lambda_{T}^{*} A_{T}+3 \operatorname{Tr}\left(Y_{d}^{\dagger} A_{d}\right)+\lambda_{S}^{*} A_{S}+\operatorname{Tr}\left(Y_{e}^{\dagger} A_{e}\right)\right)-9 A_{e} \operatorname{Tr}\left(Y_{d} Y_{d}^{\dagger} Y_{d} Y_{d}^{\dagger}\right) \\
& -3 A_{e} \operatorname{Tr}\left(Y_{d} Y_{u}^{\dagger} Y_{u} Y_{d}^{\dagger}\right)-3 A_{e} \operatorname{Tr}\left(Y_{e} Y_{e}^{\dagger} Y_{e} Y_{e}^{\dagger}\right) \\
& -\frac{2}{5} Y_{e}\left(135 g_{1}^{4} M_{1}+9 g_{1}^{2} g_{2}^{2} M_{1}+9 g_{1}^{2} g_{2}^{2} M_{2}+135 g_{2}^{4} M_{2}+30 \lambda_{S} \lambda_{S}^{*, 2} A_{S}\right. \\
& +10 \kappa_{S}^{*} \lambda_{S}^{*}\left(\kappa_{S} A_{S}+\lambda_{S} A_{\kappa}\right) \\
& +150 \lambda_{T} \lambda_{T}^{*, 2} A_{T}-2 g_{1}^{2} M_{1} \operatorname{Tr}\left(Y_{d} Y_{d}^{\dagger}\right)+80 g_{3}^{2} M_{3} \operatorname{Tr}\left(Y_{d} Y_{d}^{\dagger}\right)+6 g_{1}^{2} M_{1} \operatorname{Tr}\left(Y_{e} Y_{e}^{\dagger}\right) \\
& +15 \lambda_{S}^{*} A_{S} \operatorname{Tr}\left(Y_{u} Y_{u}^{\dagger}\right)+2 g_{1}^{2} \operatorname{Tr}\left(Y_{d}^{\dagger} A_{d}\right)-80 g_{3}^{2} \operatorname{Tr}\left(Y_{d}^{\dagger} A_{d}\right)-6 g_{1}^{2} \operatorname{Tr}\left(Y_{e}^{\dagger} A_{e}\right) \\
& +15\left|\lambda_{S}\right|^{2} \operatorname{Tr}\left(Y_{u}^{\dagger} A_{u}\right) \\
& +15 \lambda_{T}^{*}\left(2 \lambda_{S}^{*}\left(\lambda_{S} A_{T}+\lambda_{T} A_{S}\right)+3 A_{T} \operatorname{Tr}\left(Y_{u} Y_{u}^{\dagger}\right)+3 \lambda_{T} \operatorname{Tr}\left(Y_{u}^{\dagger} A_{u}\right)-4 g_{2}^{2} A_{T}+4 g_{2}^{2} M_{2} \lambda_{T}\right) \\
& \left.+90 \operatorname{Tr}\left(Y_{d} Y_{d}^{\dagger} A_{d} Y_{d}^{\dagger}\right)+15 \operatorname{Tr}\left(Y_{d} Y_{u}^{\dagger} A_{u} Y_{d}^{\dagger}\right)+30 \operatorname{Tr}\left(Y_{e} Y_{e}^{\dagger} A_{e} Y_{e}^{\dagger}\right)+15 \operatorname{Tr}\left(Y_{u} Y_{d}^{\dagger} A_{d} Y_{u}^{\dagger}\right)\right) \\
& \beta_{A_{T}}^{(1)}=+2 \lambda_{S}^{*}\left(2 \lambda_{T} A_{S}+\lambda_{S} A_{T}\right)+A_{T}\left(24\left|\lambda_{T}\right|^{2}+3 \operatorname{Tr}\left(Y_{d} Y_{d}^{\dagger}\right)+3 \operatorname{Tr}\left(Y_{u} Y_{u}^{\dagger}\right)\right. \\
& \left.-7 g_{2}^{2}-\frac{3}{5} g_{1}^{2}+\operatorname{Tr}\left(Y_{e} Y_{e}^{\dagger}\right)\right) \\
& +\frac{2}{5} \lambda_{T}\left(15 \operatorname{Tr}\left(Y_{d}^{\dagger} A_{d}\right)+15 \operatorname{Tr}\left(Y_{u}^{\dagger} A_{u}\right)+35 g_{2}^{2} M_{2}+3 g_{1}^{2} M_{1}+5 \operatorname{Tr}\left(Y_{e}^{\dagger} A_{e}\right)\right) \\
& \beta_{A_{T}}^{(2)}=-\frac{414}{25} g_{1}^{4} M_{1} \lambda_{T}-\frac{18}{5} g_{1}^{2} g_{2}^{2} M_{1} \lambda_{T}-\frac{18}{5} g_{1}^{2} g_{2}^{2} M_{2} \lambda_{T}-166 g_{2}^{4} M_{2} \lambda_{T}-24 \lambda_{S} \lambda_{T} \lambda_{S}^{*, 2} A_{S} \\
& +\frac{207}{50} g_{1}^{4} A_{T}+\frac{9}{5} g_{1}^{2} g_{2}^{2} A_{T}+\frac{83}{2} g_{2}^{4} A_{T}-6 \lambda_{S}^{2} \lambda_{S}^{*, 2} A_{T}-210 \lambda_{T}^{2} \lambda_{T}^{*, 2} A_{T} \\
& -4 \kappa_{S}^{*} \lambda_{S}^{*}\left(2 \kappa_{S} \lambda_{T} A_{S}+2 \lambda_{S} \lambda_{T} A_{\kappa}+\kappa_{S} \lambda_{S} A_{T}\right)+\frac{4}{5} g_{1}^{2} M_{1} \lambda_{T} \operatorname{Tr}\left(Y_{d} Y_{d}^{\dagger}\right) \\
& -32 g_{3}^{2} M_{3} \lambda_{T} \operatorname{Tr}\left(Y_{d} Y_{d}^{\dagger}\right) \\
& -6 \lambda_{T} \lambda_{S}^{*} A_{S} \operatorname{Tr}\left(Y_{d} Y_{d}^{\dagger}\right)-\frac{2}{5} g_{1}^{2} A_{T} \operatorname{Tr}\left(Y_{d} Y_{d}^{\dagger}\right)+16 g_{3}^{2} A_{T} \operatorname{Tr}\left(Y_{d} Y_{d}^{\dagger}\right)-3\left|\lambda_{S}\right|^{2} A_{T} \operatorname{Tr}\left(Y_{d} Y_{d}^{\dagger}\right) \\
& -\frac{12}{5} g_{1}^{2} M_{1} \lambda_{T} \operatorname{Tr}\left(Y_{e} Y_{e}^{\dagger}\right)-2 \lambda_{T} \lambda_{S}^{*} A_{S} \operatorname{Tr}\left(Y_{e} Y_{e}^{\dagger}\right)+\frac{6}{5} g_{1}^{2} A_{T} \operatorname{Tr}\left(Y_{e} Y_{e}^{\dagger}\right) \\
& -\left|\lambda_{S}\right|^{2} A_{T} \operatorname{Tr}\left(Y_{e} Y_{e}^{\dagger}\right)-\frac{8}{5} g_{1}^{2} M_{1} \lambda_{T} \operatorname{Tr}\left(Y_{u} Y_{u}^{\dagger}\right)-32 g_{3}^{2} M_{3} \lambda_{T} \operatorname{Tr}\left(Y_{u} Y_{u}^{\dagger}\right) \\
& -6 \lambda_{T} \lambda_{S}^{*} A_{S} \operatorname{Tr}\left(Y_{u} Y_{u}^{\dagger}\right)+\frac{4}{5} g_{1}^{2} A_{T} \operatorname{Tr}\left(Y_{u} Y_{u}^{\dagger}\right)+16 g_{3}^{2} A_{T} \operatorname{Tr}\left(Y_{u} Y_{u}^{\dagger}\right)-3\left|\lambda_{S}\right|^{2} A_{T} \operatorname{Tr}\left(Y_{u} Y_{u}^{\dagger}\right) \\
& -\frac{4}{5} g_{1}^{2} \lambda_{T} \operatorname{Tr}\left(Y_{d}^{\dagger} A_{d}\right)+32 g_{3}^{2} \lambda_{T} \operatorname{Tr}\left(Y_{d}^{\dagger} A_{d}\right)-6 \lambda_{T}\left|\lambda_{S}\right|^{2} \operatorname{Tr}\left(Y_{d}^{\dagger} A_{d}\right)+\frac{12}{5} g_{1}^{2} \lambda_{T} \operatorname{Tr}\left(Y_{e}^{\dagger} A_{e}\right)
\end{aligned}
$$




$$
\begin{aligned}
& -2 \lambda_{T}\left|\lambda_{S}\right|^{2} \operatorname{Tr}\left(Y_{e}^{\dagger} A_{e}\right)+\frac{8}{5} g_{1}^{2} \lambda_{T} \operatorname{Tr}\left(Y_{u}^{\dagger} A_{u}\right)+32 g_{3}^{2} \lambda_{T} \operatorname{Tr}\left(Y_{u}^{\dagger} A_{u}\right)-6 \lambda_{T}\left|\lambda_{S}\right|^{2} \operatorname{Tr}\left(Y_{u}^{\dagger} A_{u}\right) \\
& -\frac{1}{5}\left|\lambda_{T}\right|^{2}\left(80 \lambda_{S}^{*}\left(2 \lambda_{T} A_{S}+3 \lambda_{S} A_{T}\right)\right. \\
& -3 A_{T}\left(110 g_{2}^{2}-25 \operatorname{Tr}\left(Y_{e} Y_{e}^{\dagger}\right)+6 g_{1}^{2}-75 \operatorname{Tr}\left(Y_{d} Y_{d}^{\dagger}\right)-75 \operatorname{Tr}\left(Y_{u} Y_{u}^{\dagger}\right)\right) \\
& \left.+2 \lambda_{T}\left(110 g_{2}^{2} M_{2}+25 \operatorname{Tr}\left(Y_{e}^{\dagger} A_{e}\right)+6 g_{1}^{2} M_{1}+75 \operatorname{Tr}\left(Y_{d}^{\dagger} A_{d}\right)+75 \operatorname{Tr}\left(Y_{u}^{\dagger} A_{u}\right)\right)\right) \\
& -9 A_{T} \operatorname{Tr}\left(Y_{d} Y_{d}^{\dagger} Y_{d} Y_{d}^{\dagger}\right)-36 \lambda_{T} \operatorname{Tr}\left(Y_{d} Y_{d}^{\dagger} A_{d} Y_{d}^{\dagger}\right)-6 A_{T} \operatorname{Tr}\left(Y_{d} Y_{u}^{\dagger} Y_{u} Y_{d}^{\dagger}\right) \\
& -12 \lambda_{T} \operatorname{Tr}\left(Y_{d} Y_{u}^{\dagger} A_{u} Y_{d}^{\dagger}\right)-3 A_{T} \operatorname{Tr}\left(Y_{e} Y_{e}^{\dagger} Y_{e} Y_{e}^{\dagger}\right)-12 \lambda_{T} \operatorname{Tr}\left(Y_{e} Y_{e}^{\dagger} A_{e} Y_{e}^{\dagger}\right) \\
& -12 \lambda_{T} \operatorname{Tr}\left(Y_{u} Y_{d}^{\dagger} A_{d} Y_{u}^{\dagger}\right)-9 A_{T} \operatorname{Tr}\left(Y_{u} Y_{u}^{\dagger} Y_{u} Y_{u}^{\dagger}\right)-36 \lambda_{T} \operatorname{Tr}\left(Y_{u} Y_{u}^{\dagger} A_{u} Y_{u}^{\dagger}\right) \\
& \beta_{A_{S}}^{(1)}=+2 \kappa_{S}^{*}\left(2 \lambda_{S} A_{\kappa}+\kappa_{S} A_{S}\right) \\
& +A_{S}\left(12\left|\lambda_{S}\right|^{2}-3 g_{2}^{2}+3 \operatorname{Tr}\left(Y_{d} Y_{d}^{\dagger}\right)+3 \operatorname{Tr}\left(Y_{u} Y_{u}^{\dagger}\right)+6\left|\lambda_{T}\right|^{2}-\frac{3}{5} g_{1}^{2}+\operatorname{Tr}\left(Y_{e} Y_{e}^{\dagger}\right)\right) \\
& +\frac{2}{5} \lambda_{S}\left(15 g_{2}^{2} M_{2}+15 \operatorname{Tr}\left(Y_{d}^{\dagger} A_{d}\right)+15 \operatorname{Tr}\left(Y_{u}^{\dagger} A_{u}\right)+30 \lambda_{T}^{*} A_{T}+3 g_{1}^{2} M_{1}+5 \operatorname{Tr}\left(Y_{e}^{\dagger} A_{e}\right)\right) \\
& \beta_{A_{S}}^{(2)}=-\frac{414}{25} g_{1}^{4} M_{1} \lambda_{S}-\frac{18}{5} g_{1}^{2} g_{2}^{2} M_{1} \lambda_{S}-\frac{18}{5} g_{1}^{2} g_{2}^{2} M_{2} \lambda_{S}-54 g_{2}^{4} M_{2} \lambda_{S}-32 \kappa_{S} \lambda_{S} \kappa_{S}^{*, 2} A_{\kappa} \\
& +\frac{207}{50} g_{1}^{4} A_{S}+\frac{9}{5} g_{1}^{2} g_{2}^{2} A_{S}+\frac{27}{2} g_{2}^{4} A_{S}-8 \kappa_{S}^{2} \kappa_{S}^{*, 2} A_{S}-50 \lambda_{S}^{2} \lambda_{S}^{*, 2} A_{S} \\
& -30 \lambda_{T} \lambda_{T}^{*, 2}\left(4 \lambda_{S} A_{T}+\lambda_{T} A_{S}\right) \\
& +\frac{4}{5} g_{1}^{2} M_{1} \lambda_{S} \operatorname{Tr}\left(Y_{d} Y_{d}^{\dagger}\right)-32 g_{3}^{2} M_{3} \lambda_{S} \operatorname{Tr}\left(Y_{d} Y_{d}^{\dagger}\right)-\frac{2}{5} g_{1}^{2} A_{S} \operatorname{Tr}\left(Y_{d} Y_{d}^{\dagger}\right) \\
& +16 g_{3}^{2} A_{S} \operatorname{Tr}\left(Y_{d} Y_{d}^{\dagger}\right)-\frac{12}{5} g_{1}^{2} M_{1} \lambda_{S} \operatorname{Tr}\left(Y_{e} Y_{e}^{\dagger}\right)+\frac{6}{5} g_{1}^{2} A_{S} \operatorname{Tr}\left(Y_{e} Y_{e}^{\dagger}\right)-\frac{8}{5} g_{1}^{2} M_{1} \lambda_{S} \operatorname{Tr}\left(Y_{u} Y_{u}^{\dagger}\right) \\
& -32 g_{3}^{2} M_{3} \lambda_{S} \operatorname{Tr}\left(Y_{u} Y_{u}^{\dagger}\right)+\frac{4}{5} g_{1}^{2} A_{S} \operatorname{Tr}\left(Y_{u} Y_{u}^{\dagger}\right)+16 g_{3}^{2} A_{S} \operatorname{Tr}\left(Y_{u} Y_{u}^{\dagger}\right)-\frac{4}{5} g_{1}^{2} \lambda_{S} \operatorname{Tr}\left(Y_{d}^{\dagger} A_{d}\right) \\
& +32 g_{3}^{2} \lambda_{S} \operatorname{Tr}\left(Y_{d}^{\dagger} A_{d}\right)+\frac{12}{5} g_{1}^{2} \lambda_{S} \operatorname{Tr}\left(Y_{e}^{\dagger} A_{e}\right)+\frac{8}{5} g_{1}^{2} \lambda_{S} \operatorname{Tr}\left(Y_{u}^{\dagger} A_{u}\right)+32 g_{3}^{2} \lambda_{S} \operatorname{Tr}\left(Y_{u}^{\dagger} A_{u}\right) \\
& -\frac{3}{5}\left|\lambda_{S}\right|^{2}\left(20 \kappa_{S}^{*}\left(2 \lambda_{S} A_{\kappa}+3 \kappa_{S} A_{S}\right)\right. \\
& +A_{S}\left(120 \lambda_{T} \lambda_{T}^{*}+15 \operatorname{Tr}\left(Y_{e} Y_{e}^{\dagger}\right)-30 g_{2}^{2}+45 \operatorname{Tr}\left(Y_{d} Y_{d}^{\dagger}\right)+45 \operatorname{Tr}\left(Y_{u} Y_{u}^{\dagger}\right)-6 g_{1}^{2}\right) \\
& \left.+2 \lambda_{S}\left(10 g_{2}^{2} M_{2}+15 \operatorname{Tr}\left(Y_{d}^{\dagger} A_{d}\right)+15 \operatorname{Tr}\left(Y_{u}^{\dagger} A_{u}\right)+2 g_{1}^{2} M_{1}+40 \lambda_{T}^{*} A_{T}+5 \operatorname{Tr}\left(Y_{e}^{\dagger} A_{e}\right)\right)\right) \\
& -3 \lambda_{T}^{*}\left(\lambda_{T} A_{S}\left(3 \operatorname{Tr}\left(Y_{d} Y_{d}^{\dagger}\right)+3 \operatorname{Tr}\left(Y_{u} Y_{u}^{\dagger}\right)-8 g_{2}^{2}+\operatorname{Tr}\left(Y_{e} Y_{e}^{\dagger}\right)\right)\right. \\
& +2 \lambda_{S}\left(A_{T}\left(3 \operatorname{Tr}\left(Y_{d} Y_{d}^{\dagger}\right)+3 \operatorname{Tr}\left(Y_{u} Y_{u}^{\dagger}\right)-8 g_{2}^{2}+\operatorname{Tr}\left(Y_{e} Y_{e}^{\dagger}\right)\right)\right. \\
& \left.\left.+\lambda_{T}\left(3 \operatorname{Tr}\left(Y_{d}^{\dagger} A_{d}\right)+3 \operatorname{Tr}\left(Y_{u}^{\dagger} A_{u}\right)+8 g_{2}^{2} M_{2}+\operatorname{Tr}\left(Y_{e}^{\dagger} A_{e}\right)\right)\right)\right) \\
& -9 A_{S} \operatorname{Tr}\left(Y_{d} Y_{d}^{\dagger} Y_{d} Y_{d}^{\dagger}\right)-36 \lambda_{S} \operatorname{Tr}\left(Y_{d} Y_{d}^{\dagger} A_{d} Y_{d}^{\dagger}\right)-6 A_{S} \operatorname{Tr}\left(Y_{d} Y_{u}^{\dagger} Y_{u} Y_{d}^{\dagger}\right) \\
& -12 \lambda_{S} \operatorname{Tr}\left(Y_{d} Y_{u}^{\dagger} A_{u} Y_{d}^{\dagger}\right)-3 A_{S} \operatorname{Tr}\left(Y_{e} Y_{e}^{\dagger} Y_{e} Y_{e}^{\dagger}\right)-12 \lambda_{S} \operatorname{Tr}\left(Y_{e} Y_{e}^{\dagger} A_{e} Y_{e}^{\dagger}\right) \\
& -12 \lambda_{S} \operatorname{Tr}\left(Y_{u} Y_{d}^{\dagger} A_{d} Y_{u}^{\dagger}\right)-9 A_{S} \operatorname{Tr}\left(Y_{u} Y_{u}^{\dagger} Y_{u} Y_{u}^{\dagger}\right)-36 \lambda_{S} \operatorname{Tr}\left(Y_{u} Y_{u}^{\dagger} A_{u} Y_{u}^{\dagger}\right)
\end{aligned}
$$




$$
\begin{aligned}
\beta_{A_{\kappa}}^{(1)}= & 6\left(3\left|\kappa_{S}\right|^{2} A_{\kappa}+\lambda_{S}^{*}\left(2 \kappa_{S} A_{S}+\lambda_{S} A_{\kappa}\right)\right) \\
\beta_{A_{\kappa}}^{(2)}= & -\frac{6}{5}\left(100 \kappa_{S}^{2} \kappa_{S}^{* 2} A_{\kappa}+10 \lambda_{S} \lambda_{S}^{*, 2}\left(4 \kappa_{S} A_{S}+\lambda_{S} A_{\kappa}\right)\right. \\
& +\lambda_{S}^{*}\left(\lambda _ { S } A _ { \kappa } \left(-15 g_{2}^{2}+15 \operatorname{Tr}\left(Y_{d} Y_{d}^{\dagger}\right)+15 \operatorname{Tr}\left(Y_{u} Y_{u}^{\dagger}\right)+30\left|\lambda_{T}\right|^{2}-3 g_{1}^{2}+5 \operatorname{Tr}\left(Y_{e} Y_{e}^{\dagger}\right)\right.\right. \\
& \left.+60\left|\kappa_{S}\right|^{2}\right)+2 \kappa_{S}\left(A _ { S } \left(-15 g_{2}^{2}+15 \operatorname{Tr}\left(Y_{d} Y_{d}^{\dagger}\right)+15 \operatorname{Tr}\left(Y_{u} Y_{u}^{\dagger}\right)+20\left|\kappa_{S}\right|^{2}\right.\right. \\
& \left.+30\left|\lambda_{T}\right|^{2}-3 g_{1}^{2}+5 \operatorname{Tr}\left(Y_{e} Y_{e}^{\dagger}\right)\right) \\
& \left.\left.\left.+\lambda_{S}\left(15 g_{2}^{2} M_{2}+15 \operatorname{Tr}\left(Y_{d}^{\dagger} A_{d}\right)+15 \operatorname{Tr}\left(Y_{u}^{\dagger} A_{u}\right)+30 \lambda_{T}^{*} A_{T}+3 g_{1}^{2} M_{1}+5 \operatorname{Tr}\left(Y_{e}^{\dagger} A_{e}\right)\right)\right)\right)\right)
\end{aligned}
$$

\section{B.8 Bilinear soft-breaking parameters}

$$
\begin{aligned}
& \beta_{B_{\mu}}^{(1)}=+\frac{6}{5} g_{1}^{2} M_{1} \mu+6 g_{2}^{2} M_{2} \mu+2 \lambda_{S} B_{S} \kappa_{S}^{*}+4 \mu \lambda_{S}^{*} A_{S}+12 \mu \lambda_{T}^{*} A_{T} \\
& +B_{\mu}\left(-3 g_{2}^{2}+3 \operatorname{Tr}\left(Y_{d} Y_{d}^{\dagger}\right)+3 \operatorname{Tr}\left(Y_{u} Y_{u}^{\dagger}\right)+6\left|\lambda_{S}\right|^{2}+6\left|\lambda_{T}\right|^{2}-\frac{3}{5} g_{1}^{2}+\operatorname{Tr}\left(Y_{e} Y_{e}^{\dagger}\right)\right) \\
& +6 \mu \operatorname{Tr}\left(Y_{d}^{\dagger} A_{d}\right)+2 \mu \operatorname{Tr}\left(Y_{e}^{\dagger} A_{e}\right)+6 \mu \operatorname{Tr}\left(Y_{u}^{\dagger} A_{u}\right) \\
& \beta_{B_{\mu}}^{(2)}=+B_{\mu}\left(\frac{207}{50} g_{1}^{4}+\frac{9}{5} g_{1}^{2} g_{2}^{2}+\frac{27}{2} g_{2}^{4}-14 \lambda_{S}^{2} \lambda_{S}^{*, 2}-30 \lambda_{T}^{2} \lambda_{T}^{*, 2}-\frac{2}{5} g_{1}^{2} \operatorname{Tr}\left(Y_{d} Y_{d}^{\dagger}\right)\right. \\
& +16 g_{3}^{2} \operatorname{Tr}\left(Y_{d} Y_{d}^{\dagger}\right)+\frac{6}{5} g_{1}^{2} \operatorname{Tr}\left(Y_{e} Y_{e}^{\dagger}\right) \\
& +\frac{1}{5}\left|\lambda_{S}\right|^{2}\left(180 g_{2}^{2}-180 \lambda_{T} \lambda_{T}^{*}-20 \kappa_{S} \kappa_{S}^{*}-25 \operatorname{Tr}\left(Y_{e} Y_{e}^{\dagger}\right)+36 g_{1}^{2}-75 \operatorname{Tr}\left(Y_{d} Y_{d}^{\dagger}\right)\right. \\
& \left.-75 \operatorname{Tr}\left(Y_{u} Y_{u}^{\dagger}\right)\right)+3\left|\lambda_{T}\right|^{2}\left(-3 \operatorname{Tr}\left(Y_{d} Y_{d}^{\dagger}\right)-3 \operatorname{Tr}\left(Y_{u} Y_{u}^{\dagger}\right)+8 g_{2}^{2}-\operatorname{Tr}\left(Y_{e} Y_{e}^{\dagger}\right)\right) \\
& +\frac{4}{5} g_{1}^{2} \operatorname{Tr}\left(Y_{u} Y_{u}^{\dagger}\right)+16 g_{3}^{2} \operatorname{Tr}\left(Y_{u} Y_{u}^{\dagger}\right)-9 \operatorname{Tr}\left(Y_{d} Y_{d}^{\dagger} Y_{d} Y_{d}^{\dagger}\right)-6 \operatorname{Tr}\left(Y_{d} Y_{u}^{\dagger} Y_{u} Y_{d}^{\dagger}\right) \\
& \left.-3 \operatorname{Tr}\left(Y_{e} Y_{e}^{\dagger} Y_{e} Y_{e}^{\dagger}\right)-9 \operatorname{Tr}\left(Y_{u} Y_{u}^{\dagger} Y_{u} Y_{u}^{\dagger}\right)\right) \\
& -\frac{2}{25}\left(207 g_{1}^{4} M_{1} \mu+45 g_{1}^{2} g_{2}^{2} M_{1} \mu+45 g_{1}^{2} g_{2}^{2} M_{2} \mu+675 g_{2}^{4} M_{2} \mu+600 g_{2}^{2} M_{2} \mu\left|\lambda_{T}\right|^{2}\right. \\
& +100 \lambda_{S}\left(\left|\kappa_{S}\right|^{2}+\left|\lambda_{S}\right|^{2}\right) B_{S} \kappa_{S}^{*}+100 M_{S} \lambda_{S} \kappa_{S}^{*, 2} A_{\kappa}+400 \lambda_{S} \mu \lambda_{S}^{*, 2} A_{S}-600 g_{2}^{2} \mu \lambda_{T}^{*} A_{T} \\
& +1500 \lambda_{T} \mu \lambda_{T}^{*, 2} A_{T}-10 g_{1}^{2} M_{1} \mu \operatorname{Tr}\left(Y_{d} Y_{d}^{\dagger}\right)+400 g_{3}^{2} M_{3} \mu \operatorname{Tr}\left(Y_{d} Y_{d}^{\dagger}\right)+225 \mu \lambda_{T}^{*} A_{T} \operatorname{Tr}\left(Y_{d} Y_{d}^{\dagger}\right) \\
& +30 g_{1}^{2} M_{1} \mu \operatorname{Tr}\left(Y_{e} Y_{e}^{\dagger}\right)+75 \mu \lambda_{T}^{*} A_{T} \operatorname{Tr}\left(Y_{e} Y_{e}^{\dagger}\right)+20 g_{1}^{2} M_{1} \mu \operatorname{Tr}\left(Y_{u} Y_{u}^{\dagger}\right) \\
& +400 g_{3}^{2} M_{3} \mu \operatorname{Tr}\left(Y_{u} Y_{u}^{\dagger}\right)+225 \mu \lambda_{T}^{*} A_{T} \operatorname{Tr}\left(Y_{u} Y_{u}^{\dagger}\right)+10 g_{1}^{2} \mu \operatorname{Tr}\left(Y_{d}^{\dagger} A_{d}\right)-400 g_{3}^{2} \mu \operatorname{Tr}\left(Y_{d}^{\dagger} A_{d}\right) \\
& +225 \mu\left|\lambda_{T}\right|^{2} \operatorname{Tr}\left(Y_{d}^{\dagger} A_{d}\right)-30 g_{1}^{2} \mu \operatorname{Tr}\left(Y_{e}^{\dagger} A_{e}\right)+75 \mu\left|\lambda_{T}\right|^{2} \operatorname{Tr}\left(Y_{e}^{\dagger} A_{e}\right)-20 g_{1}^{2} \mu \operatorname{Tr}\left(Y_{u}^{\dagger} A_{u}\right) \\
& -400 g_{3}^{2} \mu \operatorname{Tr}\left(Y_{u}^{\dagger} A_{u}\right)+225 \mu\left|\lambda_{T}\right|^{2} \operatorname{Tr}\left(Y_{u}^{\dagger} A_{u}\right) \\
& +5 \lambda_{S}^{*}\left(20 \kappa_{S}^{*}\left(\left(\kappa_{S} \mu+M_{S} \lambda_{S}\right) A_{S}+\lambda_{S} \mu A_{\kappa}\right)\right. \\
& +\mu\left(60 \lambda_{T}^{*}\left(2 \lambda_{S} A_{T}+\lambda_{T} A_{S}\right)+5 A_{S}\left(3 \operatorname{Tr}\left(Y_{d} Y_{d}^{\dagger}\right)+3 \operatorname{Tr}\left(Y_{u} Y_{u}^{\dagger}\right)+\operatorname{Tr}\left(Y_{e} Y_{e}^{\dagger}\right)\right)\right. \\
& \left.\left.+3 \lambda_{S}\left(15 \operatorname{Tr}\left(Y_{d}^{\dagger} A_{d}\right)+15 \operatorname{Tr}\left(Y_{u}^{\dagger} A_{u}\right)+30 g_{2}^{2} M_{2}+5 \operatorname{Tr}\left(Y_{e}^{\dagger} A_{e}\right)+6 g_{1}^{2} M_{1}\right)\right)\right)
\end{aligned}
$$




$$
\begin{aligned}
& +450 \mu \operatorname{Tr}\left(Y_{d} Y_{d}^{\dagger} A_{d} Y_{d}^{\dagger}\right)+150 \mu \operatorname{Tr}\left(Y_{d} Y_{u}^{\dagger} A_{u} Y_{d}^{\dagger}\right)+150 \mu \operatorname{Tr}\left(Y_{e} Y_{e}^{\dagger} A_{e} Y_{e}^{\dagger}\right) \\
& \left.+150 \mu \operatorname{Tr}\left(Y_{u} Y_{d}^{\dagger} A_{d} Y_{u}^{\dagger}\right)+450 \mu \operatorname{Tr}\left(Y_{u} Y_{u}^{\dagger} A_{u} Y_{u}^{\dagger}\right)\right) \\
& \beta_{B_{T}}^{(1)}=4\left(-2 g_{2}^{2} B_{T}+2 M_{T} \lambda_{T}^{*} A_{T}+4 g_{2}^{2} M_{2} M_{T}+\left|\lambda_{T}\right|^{2} B_{T}\right) \\
& \beta_{B_{T}}^{(2)}=-\frac{4}{5}\left(B _ { T } \left(30 \lambda_{T}^{2} \lambda_{T}^{*, 2}-70 g_{2}^{4}\right.\right. \\
& \left.+\left|\lambda_{T}\right|^{2}\left(10 \lambda_{S} \lambda_{S}^{*}+15 \operatorname{Tr}\left(Y_{d} Y_{d}^{\dagger}\right)+15 \operatorname{Tr}\left(Y_{u} Y_{u}^{\dagger}\right)-3 g_{1}^{2}+5 g_{2}^{2}+5 \operatorname{Tr}\left(Y_{e} Y_{e}^{\dagger}\right)\right)\right) \\
& +2 M_{T}\left(140 g_{2}^{4} M_{2}+60 \lambda_{T} \lambda_{T}^{*, 2} A_{T}+\lambda_{T}^{*}\left(10 \lambda_{S}^{*}\left(\lambda_{S} A_{T}+\lambda_{T} A_{S}\right)\right.\right. \\
& +A_{T}\left(15 \operatorname{Tr}\left(Y_{d} Y_{d}^{\dagger}\right)+15 \operatorname{Tr}\left(Y_{u} Y_{u}^{\dagger}\right)-3 g_{1}^{2}+5 g_{2}^{2}+5 \operatorname{Tr}\left(Y_{e} Y_{e}^{\dagger}\right)\right) \\
& \left.\left.\left.+\lambda_{T}\left(15 \operatorname{Tr}\left(Y_{d}^{\dagger} A_{d}\right)+15 \operatorname{Tr}\left(Y_{u}^{\dagger} A_{u}\right)+3 g_{1}^{2} M_{1}-5 g_{2}^{2} M_{2}+5 \operatorname{Tr}\left(Y_{e}^{\dagger} A_{e}\right)\right)\right)\right)\right) \\
& \beta_{B_{O}}^{(1)}=12 g_{3}^{2}\left(2 M_{3} M_{O}-B_{O}\right) \\
& \beta_{B_{O}}^{(2)}=72 g_{3}^{4}\left(-4 M_{3} M_{O}+B_{O}\right) \\
& \beta_{B_{S}}^{(1)}=4\left(2\left|\kappa_{S}\right|^{2}+\left|\lambda_{S}\right|^{2}\right) B_{S}+8\left(\kappa_{S} B_{\mu} \lambda_{S}^{*}+M_{S} \kappa_{S}^{*} A_{\kappa}+M_{S} \lambda_{S}^{*} A_{S}\right) \\
& \beta_{B_{S}}^{(2)}=-\frac{4}{5}\left(B _ { S } \left(40 \kappa_{S}^{2} \kappa_{S}^{*, 2}+40 \lambda_{S}\left|\kappa_{S}\right|^{2} \lambda_{S}^{*}\right.\right. \\
& \left.+\left|\lambda_{S}\right|^{2}\left(10 \lambda_{S} \lambda_{S}^{*}-15 g_{2}^{2}+15 \operatorname{Tr}\left(Y_{d} Y_{d}^{\dagger}\right)+15 \operatorname{Tr}\left(Y_{u} Y_{u}^{\dagger}\right)+30 \lambda_{T} \lambda_{T}^{*}-3 g_{1}^{2}+5 \operatorname{Tr}\left(Y_{e} Y_{e}^{\dagger}\right)\right)\right) \\
& +2\left(50 M_{S} \kappa_{S} \kappa_{S}^{*, 2} A_{\kappa}+10 \lambda_{S}^{*, 2}\left(\left(2 M_{S} \lambda_{S}+\kappa_{S} \mu\right) A_{S}+\kappa_{S} \lambda_{S} B_{\mu}\right)\right. \\
& +\lambda_{S}^{*}\left(3 g_{1}^{2} M_{1} M_{S} \lambda_{S}+15 g_{2}^{2} M_{2} M_{S} \lambda_{S}+9 g_{1}^{2} M_{1} \kappa_{S} \mu+45 g_{2}^{2} M_{2} \kappa_{S} \mu-3 g_{1}^{2} M_{S} A_{S}\right. \\
& -15 g_{2}^{2} M_{S} A_{S} \\
& +30 M_{S}\left|\lambda_{T}\right|^{2} A_{S}+10 M_{S} \kappa_{S}^{*}\left(2 \lambda_{S} A_{\kappa}+3 \kappa_{S} A_{S}\right)+30 M_{S} \lambda_{S} \lambda_{T}^{*} A_{T}+30 \kappa_{S} \mu \lambda_{T}^{*} A_{T} \\
& +15 M_{S} A_{S} \operatorname{Tr}\left(Y_{d} Y_{d}^{\dagger}\right)+5 M_{S} A_{S} \operatorname{Tr}\left(Y_{e} Y_{e}^{\dagger}\right)+15 M_{S} A_{S} \operatorname{Tr}\left(Y_{u} Y_{u}^{\dagger}\right)+\kappa_{S} B_{\mu}\left(15 \operatorname{Tr}\left(Y_{d} Y_{d}^{\dagger}\right)\right. \\
& \left.+15 \operatorname{Tr}\left(Y_{u} Y_{u}^{\dagger}\right)+30\left|\lambda_{T}\right|^{2}-45 g_{2}^{2}+5 \operatorname{Tr}\left(Y_{e} Y_{e}^{\dagger}\right)-9 g_{1}^{2}\right)+15 M_{S} \lambda_{S} \operatorname{Tr}\left(Y_{d}^{\dagger} A_{d}\right) \\
& +15 \kappa_{S} \mu \operatorname{Tr}\left(Y_{d}^{\dagger} A_{d}\right)+5 M_{S} \lambda_{S} \operatorname{Tr}\left(Y_{e}^{\dagger} A_{e}\right)+5 \kappa_{S} \mu \operatorname{Tr}\left(Y_{e}^{\dagger} A_{e}\right)+15 M_{S} \lambda_{S} \operatorname{Tr}\left(Y_{u}^{\dagger} A_{u}\right) \\
& \left.\left.\left.+15 \kappa_{S} \mu \operatorname{Tr}\left(Y_{u}^{\dagger} A_{u}\right)\right)\right)\right)
\end{aligned}
$$

\section{B.9 Linear soft-breaking parameters}

$$
\begin{aligned}
(4 \pi)^{2} X_{S}^{(1)}= & 2\left(2 m_{S}^{2} \kappa_{S} M_{S}^{*}+M_{S} B_{S} \kappa_{S}^{*}+2 M_{S} B_{\mu} \lambda_{S}^{*}+2 m_{H_{d}}^{2} \lambda_{S} \mu^{*}+2 m_{H_{u}}^{2} \lambda_{S} \mu^{*}+\left|\kappa_{S}\right|^{2} t_{S}\right. \\
& \left.+\left|\lambda_{S}\right|^{2} t_{S}+2 L_{S} \kappa_{S}^{*} A_{\kappa}+B_{S}^{*} A_{\kappa}+2 L_{S} \lambda_{S}^{*} A_{S}+2 B_{\mu}^{*} A_{S}\right) \\
(4 \pi)^{4} X_{S}^{(2)}= & -\frac{2}{5}\left(6 g_{1}^{2} L_{S} M_{1}\left|\lambda_{S}\right|^{2}+30 g_{2}^{2} L_{S} M_{2}\left|\lambda_{S}\right|^{2}+20 M_{S}\left(\left|\kappa_{S}\right|^{2}+\left|\lambda_{S}\right|^{2}\right) B_{S} \kappa_{S}^{*}\right. \\
& +6 g_{1}^{2} M_{1} M_{S} \mu \lambda_{S}^{*}+30 g_{2}^{2} M_{2} M_{S} \mu \lambda_{S}^{*}-6 g_{1}^{2} M_{S} B_{\mu} \lambda_{S}^{*}-30 g_{2}^{2} M_{S} B_{\mu} \lambda_{S}^{*} \\
& +60 M_{S}\left|\lambda_{T}\right|^{2} B_{\mu} \lambda_{S}^{*}+20 M_{S} \lambda_{S} B_{\mu} \lambda_{S}^{*, 2}
\end{aligned}
$$


$-6 g_{1}^{2} m_{H_{d}}^{2} \lambda_{S} \mu^{*}-30 g_{2}^{2} m_{H_{d}}^{2} \lambda_{S} \mu^{*}-6 g_{1}^{2} m_{H_{u}}^{2} \lambda_{S} \mu^{*}-30 g_{2}^{2} m_{H_{u}}^{2} \lambda_{S} \mu^{*}$

$-12 g_{1}^{2} \lambda_{S}\left|M_{1}\right|^{2} \mu^{*}-60 g_{2}^{2} \lambda_{S}\left|M_{2}\right|^{2} \mu^{*}+120 m_{H_{d}}^{2} \lambda_{S}\left|\lambda_{T}\right|^{2} \mu^{*}+120 m_{H_{u}}^{2} \lambda_{S}\left|\lambda_{T}\right|^{2} \mu^{*}$

$+60 m_{T}^{2} \lambda_{S}\left|\lambda_{T}\right|^{2} \mu^{*}+40 \lambda_{S}\left|A_{S}\right|^{2} \mu^{*}+60 \lambda_{S}\left|A_{T}\right|^{2} \mu^{*}$

$+40 m_{H_{d}}^{2} \lambda_{S}^{2} \lambda_{S}^{*} \mu^{*}+40 m_{H_{u}}^{2} \lambda_{S}^{2} \lambda_{S}^{*} \mu^{*}$

$+20 m_{S}^{2} \lambda_{S}^{2} \lambda_{S}^{*} \mu^{*}+6 g_{1}^{2} M_{1} \lambda_{S} B_{\mu}^{*}+30 g_{2}^{2} M_{2} \lambda_{S} B_{\mu}^{*}-3 g_{1}^{2}\left|\lambda_{S}\right|^{2} t_{S}-15 g_{2}^{2}\left|\lambda_{S}\right|^{2} t_{S}$

$+20 \kappa_{S}^{2} \kappa_{S}^{*, 2} t_{S}+20 \lambda_{S}\left|\kappa_{S}\right|^{2} \lambda_{S}^{*} t_{S}+10 \lambda_{S}^{2} \lambda_{S}^{*, 2} t_{S}+30 \lambda_{T}\left|\lambda_{S}\right|^{2} \lambda_{T}^{*} t_{S}$

$+40 L_{S}\left|\lambda_{S}\right|^{2} \kappa_{S}^{*} A_{\kappa}+20 M_{S}^{2} \kappa_{S}^{*, 2} A_{\kappa}+80 L_{S} \kappa_{S} \kappa_{S}^{*, 2} A_{\kappa}$

$+40\left|\kappa_{S}\right|^{2} B_{S}^{*} A_{\kappa}+20\left|\lambda_{S}\right|^{2} B_{S}^{*} A_{\kappa}$

$+20 M_{S}^{*}\left(2 \kappa_{S}\left|A_{\kappa}\right|^{2}+\left(3 m_{S}^{2}+m_{H_{d}}^{2}+m_{H_{u}}^{2}\right) \kappa_{S}\left|\lambda_{S}\right|^{2}+5 m_{S}^{2} \kappa_{S}^{2} \kappa_{S}^{*}\right.$

$\left.+\kappa_{S}\left|A_{S}\right|^{2}+\lambda_{S} A_{S}^{*} A_{\kappa}\right)-6 g_{1}^{2} L_{S} \lambda_{S}^{*} A_{S}$

$-30 g_{2}^{2} L_{S} \lambda_{S}^{*} A_{S}+40 L_{S}\left|\kappa_{S}\right|^{2} \lambda_{S}^{*} A_{S}+60 L_{S}\left|\lambda_{T}\right|^{2} \lambda_{S}^{*} A_{S}+20 M_{S}^{2} \kappa_{S}^{*} \lambda_{S}^{*} A_{S}$

$+40 L_{S} \lambda_{S} \lambda_{S}^{*, 2} A_{S}+20 M_{S} \mu \lambda_{S}^{*, 2} A_{S}+6 g_{1}^{2} M_{1} \mu^{*} A_{S}+30 g_{2}^{2} M_{2} \mu^{*} A_{S}+20 \kappa_{S} \lambda_{S}^{*} B_{S}^{*} A_{S}$

$-6 g_{1}^{2} B_{\mu}^{*} A_{S}-30 g_{2}^{2} B_{\mu}^{*} A_{S}+40\left|\lambda_{S}\right|^{2} B_{\mu}^{*} A_{S}+60\left|\lambda_{T}\right|^{2} B_{\mu}^{*} A_{S}+60 \lambda_{T} \mu^{*} A_{T}^{*} A_{S}$

$+60 L_{S}\left|\lambda_{S}\right|^{2} \lambda_{T}^{*} A_{T}+60 M_{S} \mu \lambda_{S}^{*} \lambda_{T}^{*} A_{T}+60 \lambda_{S} \lambda_{T}^{*} B_{\mu}^{*} A_{T}+30 M_{S} B_{\mu} \lambda_{S}^{*} \operatorname{Tr}\left(Y_{d} Y_{d}^{\dagger}\right)$

$+60 m_{H_{d}}^{2} \lambda_{S} \mu^{*} \operatorname{Tr}\left(Y_{d} Y_{d}^{\dagger}\right)+30 m_{H_{u}}^{2} \lambda_{S} \mu^{*} \operatorname{Tr}\left(Y_{d} Y_{d}^{\dagger}\right)+15\left|\lambda_{S}\right|^{2} t_{S} \operatorname{Tr}\left(Y_{d} Y_{d}^{\dagger}\right)$

$+30 L_{S} \lambda_{S}^{*} A_{S} \operatorname{Tr}\left(Y_{d} Y_{d}^{\dagger}\right)+30 B_{\mu}^{*} A_{S} \operatorname{Tr}\left(Y_{d} Y_{d}^{\dagger}\right)+10 M_{S} B_{\mu} \lambda_{S}^{*} \operatorname{Tr}\left(Y_{e} Y_{e}^{\dagger}\right)$

$+20 m_{H_{d}}^{2} \lambda_{S} \mu^{*} \operatorname{Tr}\left(Y_{e} Y_{e}^{\dagger}\right)+10 m_{H_{u}}^{2} \lambda_{S} \mu^{*} \operatorname{Tr}\left(Y_{e} Y_{e}^{\dagger}\right)+5\left|\lambda_{S}\right|^{2} t_{S} \operatorname{Tr}\left(Y_{e} Y_{e}^{\dagger}\right)$

$+10 L_{S} \lambda_{S}^{*} A_{S} \operatorname{Tr}\left(Y_{e} Y_{e}^{\dagger}\right)+10 B_{\mu}^{*} A_{S} \operatorname{Tr}\left(Y_{e} Y_{e}^{\dagger}\right)+30 M_{S} B_{\mu} \lambda_{S}^{*} \operatorname{Tr}\left(Y_{u} Y_{u}^{\dagger}\right)$

$+30 m_{H_{d}}^{2} \lambda_{S} \mu^{*} \operatorname{Tr}\left(Y_{u} Y_{u}^{\dagger}\right)+60 m_{H_{u}}^{2} \lambda_{S} \mu^{*} \operatorname{Tr}\left(Y_{u} Y_{u}^{\dagger}\right)+15\left|\lambda_{S}\right|^{2} t_{S} \operatorname{Tr}\left(Y_{u} Y_{u}^{\dagger}\right)$

$+30 L_{S} \lambda_{S}^{*} A_{S} \operatorname{Tr}\left(Y_{u} Y_{u}^{\dagger}\right)+30 B_{\mu}^{*} A_{S} \operatorname{Tr}\left(Y_{u} Y_{u}^{\dagger}\right)$

$+30 L_{S}\left|\lambda_{S}\right|^{2} \operatorname{Tr}\left(Y_{d}^{\dagger} A_{d}\right)+30 M_{S} \mu \lambda_{S}^{*} \operatorname{Tr}\left(Y_{d}^{\dagger} A_{d}\right)$

$+30 \lambda_{S} B_{\mu}^{*} \operatorname{Tr}\left(Y_{d}^{\dagger} A_{d}\right)+10 L_{S}\left|\lambda_{S}\right|^{2} \operatorname{Tr}\left(Y_{e}^{\dagger} A_{e}\right)$

$+10 M_{S} \mu \lambda_{S}^{*} \operatorname{Tr}\left(Y_{e}^{\dagger} A_{e}\right)+10 \lambda_{S} B_{\mu}^{*} \operatorname{Tr}\left(Y_{e}^{\dagger} A_{e}\right)$

$+30 L_{S}\left|\lambda_{S}\right|^{2} \operatorname{Tr}\left(Y_{u}^{\dagger} A_{u}\right)+30 M_{S} \mu \lambda_{S}^{*} \operatorname{Tr}\left(Y_{u}^{\dagger} A_{u}\right)$

$+30 \lambda_{S} B_{\mu}^{*} \operatorname{Tr}\left(Y_{u}^{\dagger} A_{u}\right)+30 \mu^{*} A_{S} \operatorname{Tr}\left(A_{d}^{*} Y_{d}^{T}\right)$

$+30 \lambda_{S} \mu^{*} \operatorname{Tr}\left(A_{d}^{*} A_{d}^{T}\right)+10 \mu^{*} A_{S} \operatorname{Tr}\left(A_{e}^{*} Y_{e}^{T}\right)$

$+10 \lambda_{S} \mu^{*} \operatorname{Tr}\left(A_{e}^{*} A_{e}^{T}\right)+30 \mu^{*} A_{S} \operatorname{Tr}\left(A_{u}^{*} Y_{u}^{T}\right)$

$+30 \lambda_{S} \mu^{*} \operatorname{Tr}\left(A_{u}^{*} A_{u}^{T}\right)+30 \lambda_{S} \mu^{*} \operatorname{Tr}\left(Y_{d} Y_{d}^{\dagger} m_{d}^{2 *}\right)+30 \lambda_{S} \mu^{*} \operatorname{Tr}\left(Y_{d} m_{q}^{2 *} Y_{d}^{\dagger}\right)$ 


$$
\begin{aligned}
& +10 \lambda_{S} \mu^{*} \operatorname{Tr}\left(Y_{e} Y_{e}^{\dagger} m_{e}^{2 *}\right)+10 \lambda_{S} \mu^{*} \operatorname{Tr}\left(Y_{e} m_{l}^{2 *} Y_{e}^{\dagger}\right)+30 \lambda_{S} \mu^{*} \operatorname{Tr}\left(Y_{u} Y_{u}^{\dagger} m_{u}^{2 *}\right) \\
& \left.+30 \lambda_{S} \mu^{*} \operatorname{Tr}\left(Y_{u} m_{q}^{2 *} Y_{u}^{\dagger}\right)\right)
\end{aligned}
$$

\section{B.10 Soft-breaking scalar masses}

$$
\begin{aligned}
& \sigma_{1,1}=\sqrt{\frac{3}{5}} g_{1}\left(-2 \operatorname{Tr}\left(m_{u}^{2}\right)-m_{H_{d}}^{2}-\operatorname{Tr}\left(m_{l}^{2}\right)+m_{H_{u}}^{2}+\operatorname{Tr}\left(m_{q}^{2}\right)+\operatorname{Tr}\left(m_{d}^{2}\right)+\operatorname{Tr}\left(m_{e}^{2}\right)\right) \\
& \operatorname{Tr} 2 \mathrm{U} 1(1,1)=\frac{1}{10} g_{1}^{2}\left(2 \operatorname{Tr}\left(m_{d}^{2}\right)+3 m_{H_{d}}^{2}+3 m_{H_{u}}^{2}+3 \operatorname{Tr}\left(m_{l}^{2}\right)+6 \operatorname{Tr}\left(m_{e}^{2}\right)+8 \operatorname{Tr}\left(m_{u}^{2}\right)+\operatorname{Tr}\left(m_{q}^{2}\right)\right) \\
& \sigma_{3,1}=\frac{1}{20} \frac{1}{\sqrt{15}} g_{1}\left(-9 g_{1}^{2} m_{H_{d}}^{2}-45 g_{2}^{2} m_{H_{d}}^{2}+9 g_{1}^{2} m_{H_{u}}^{2}+45 g_{2}^{2} m_{H_{u}}^{2}\right. \\
& +30\left(-m_{H_{u}}^{2}+m_{H_{d}}^{2}\right)\left|\lambda_{S}\right|^{2}+90\left(-m_{H_{u}}^{2}+m_{H_{d}}^{2}\right)\left|\lambda_{T}\right|^{2} \\
& -9 g_{1}^{2} \operatorname{Tr}\left(m_{l}^{2}\right)-45 g_{2}^{2} \operatorname{Tr}\left(m_{l}^{2}\right)+g_{1}^{2} \operatorname{Tr}\left(m_{q}^{2}\right)+45 g_{2}^{2} \operatorname{Tr}\left(m_{q}^{2}\right)+80 g_{3}^{2} \operatorname{Tr}\left(m_{q}^{2}\right) \\
& +4 g_{1}^{2} \operatorname{Tr}\left(m_{d}^{2}\right)+80 g_{3}^{2} \operatorname{Tr}\left(m_{d}^{2}\right) \\
& +36 g_{1}^{2} \operatorname{Tr}\left(m_{e}^{2}\right)-32 g_{1}^{2} \operatorname{Tr}\left(m_{u}^{2}\right)-160 g_{3}^{2} \operatorname{Tr}\left(m_{u}^{2}\right)+90 m_{H_{d}}^{2} \operatorname{Tr}\left(Y_{d} Y_{d}^{\dagger}\right) \\
& +30 m_{H_{d}}^{2} \operatorname{Tr}\left(Y_{e} Y_{e}^{\dagger}\right)-90 m_{H_{u}}^{2} \operatorname{Tr}\left(Y_{u} Y_{u}^{\dagger}\right)-60 \operatorname{Tr}\left(Y_{d} Y_{d}^{\dagger} m_{d}^{2 *}\right)-30 \operatorname{Tr}\left(Y_{d} m_{q}^{2 *} Y_{d}^{\dagger}\right) \\
& \left.-60 \operatorname{Tr}\left(Y_{e} Y_{e}^{\dagger} m_{e}^{2 *}\right)+30 \operatorname{Tr}\left(Y_{e} m_{l}^{2 *} Y_{e}^{\dagger}\right)+120 \operatorname{Tr}\left(Y_{u} Y_{u}^{\dagger} m_{u}^{2 *}\right)-30 \operatorname{Tr}\left(Y_{u} m_{q}^{2 *} Y_{u}^{\dagger}\right)\right) \\
& \sigma_{2,2}=\frac{1}{2}\left(3 \operatorname{Tr}\left(m_{q}^{2}\right)+6 m_{T}^{2}+m_{H_{d}}^{2}+m_{H_{u}}^{2}+\operatorname{Tr}\left(m_{l}^{2}\right)\right) \\
& \sigma_{2,3}=\frac{1}{2}\left(16 m_{O}^{2}+2 \operatorname{Tr}\left(m_{q}^{2}\right)+\operatorname{Tr}\left(m_{d}^{2}\right)+\operatorname{Tr}\left(m_{u}^{2}\right)\right) \\
& \beta_{m_{q}^{2}}^{(1)}=-\frac{2}{15} g_{1}^{2} \mathbf{1}\left|M_{1}\right|^{2}-\frac{32}{3} g_{3}^{2} \mathbf{1}\left|M_{3}\right|^{2}-6 g_{2}^{2} \mathbf{1}\left|M_{2}\right|^{2}+2 m_{H_{d}}^{2} Y_{d}^{\dagger} Y_{d}+2 m_{H_{u}}^{2} Y_{u}^{\dagger} Y_{u}+2 A_{d}^{\dagger} A_{d} \\
& +2 A_{u}^{\dagger} A_{u}+m_{q}^{2} Y_{d}^{\dagger} Y_{d}+m_{q}^{2} Y_{u}^{\dagger} Y_{u}+2 Y_{d}^{\dagger} m_{d}^{2} Y_{d}+Y_{d}^{\dagger} Y_{d} m_{q}^{2}+2 Y_{u}^{\dagger} m_{u}^{2} Y_{u} \\
& +Y_{u}^{\dagger} Y_{u} m_{q}^{2}+\frac{1}{\sqrt{15}} g_{1} \mathbf{1} \sigma_{1,1} \\
& \beta_{m_{q}^{2}}^{(2)}=+\frac{2}{5} g_{1}^{2} g_{2}^{2} \mathbf{1}\left|M_{2}\right|^{2}+69 g_{2}^{4} \mathbf{1}\left|M_{2}\right|^{2}+32 g_{2}^{2} g_{3}^{2} \mathbf{1}\left|M_{2}\right|^{2} \\
& +\frac{16}{45} g_{3}^{2}\left(15\left(10 g_{3}^{2} M_{3}+3 g_{2}^{2}\left(2 M_{3}+M_{2}\right)\right)+g_{1}^{2}\left(2 M_{3}+M_{1}\right)\right) \mathbf{1} M_{3}^{*} \\
& +\frac{1}{5} g_{1}^{2} g_{2}^{2} M_{1} \mathbf{1} M_{2}^{*}+16 g_{2}^{2} g_{3}^{2} M_{3} \mathbf{1} M_{2}^{*} \\
& +\frac{4}{5} g_{1}^{2} m_{H_{d}}^{2} Y_{d}^{\dagger} Y_{d}-4 m_{H_{d}}^{2}\left|\lambda_{S}\right|^{2} Y_{d}^{\dagger} Y_{d}-2 m_{H_{u}}^{2}\left|\lambda_{S}\right|^{2} Y_{d}^{\dagger} Y_{d} \\
& -2 m_{S}^{2}\left|\lambda_{S}\right|^{2} Y_{d}^{\dagger} Y_{d}-12 m_{H_{d}}^{2}\left|\lambda_{T}\right|^{2} Y_{d}^{\dagger} Y_{d}-6 m_{H_{u}}^{2}\left|\lambda_{T}\right|^{2} Y_{d}^{\dagger} Y_{d} \\
& -6 m_{T}^{2}\left|\lambda_{T}\right|^{2} Y_{d}^{\dagger} Y_{d}-2\left|A_{S}\right|^{2} Y_{d}^{\dagger} Y_{d}-6\left|A_{T}\right|^{2} Y_{d}^{\dagger} Y_{d}-2 \lambda_{S} A_{S}^{*} Y_{d}^{\dagger} A_{d} \\
& -6 \lambda_{T} A_{T}^{*} Y_{d}^{\dagger} A_{d}+\frac{8}{5} g_{1}^{2} m_{H_{u}}^{2} Y_{u}^{\dagger} Y_{u}-2 m_{H_{d}}^{2}\left|\lambda_{S}\right|^{2} Y_{u}^{\dagger} Y_{u}-4 m_{H_{u}}^{2}\left|\lambda_{S}\right|^{2} Y_{u}^{\dagger} Y_{u} \\
& -2 m_{S}^{2}\left|\lambda_{S}\right|^{2} Y_{u}^{\dagger} Y_{u}-6 m_{H_{d}}^{2}\left|\lambda_{T}\right|^{2} Y_{u}^{\dagger} Y_{u}-12 m_{H_{u}}^{2}\left|\lambda_{T}\right|^{2} Y_{u}^{\dagger} Y_{u}
\end{aligned}
$$




$$
\begin{aligned}
& -6 m_{T}^{2}\left|\lambda_{T}\right|^{2} Y_{u}^{\dagger} Y_{u}-2\left|A_{S}\right|^{2} Y_{u}^{\dagger} Y_{u}-6\left|A_{T}\right|^{2} Y_{u}^{\dagger} Y_{u} \\
& +\frac{1}{225} g_{1}^{2} M_{1}^{*}\left(\left(5\left(16 g_{3}^{2}\left(2 M_{1}+M_{3}\right)+9 g_{2}^{2}\left(2 M_{1}+M_{2}\right)\right)+597 g_{1}^{2} M_{1}\right) \mathbf{1}\right. \\
& \left.+180\left(2 M_{1} Y_{d}^{\dagger} Y_{d}-2 Y_{u}^{\dagger} A_{u}+4 M_{1} Y_{u}^{\dagger} Y_{u}-Y_{d}^{\dagger} A_{d}\right)\right) \\
& -2 \lambda_{S} A_{S}^{*} Y_{u}^{\dagger} A_{u}-6 \lambda_{T} A_{T}^{*} Y_{u}^{\dagger} A_{u}-\frac{4}{5} g_{1}^{2} M_{1} A_{d}^{\dagger} Y_{d}+\frac{4}{5} g_{1}^{2} A_{d}^{\dagger} A_{d} \\
& -2\left|\lambda_{S}\right|^{2} A_{d}^{\dagger} A_{d}-6\left|\lambda_{T}\right|^{2} A_{d}^{\dagger} A_{d}-\frac{8}{5} g_{1}^{2} M_{1} A_{u}^{\dagger} Y_{u}+\frac{8}{5} g_{1}^{2} A_{u}^{\dagger} A_{u} \\
& -2\left|\lambda_{S}\right|^{2} A_{u}^{\dagger} A_{u}-6\left|\lambda_{T}\right|^{2} A_{u}^{\dagger} A_{u}+\frac{2}{5} g_{1}^{2} m_{q}^{2} Y_{d}^{\dagger} Y_{d}-\left|\lambda_{S}\right|^{2} m_{q}^{2} Y_{d}^{\dagger} Y_{d} \\
& -3\left|\lambda_{T}\right|^{2} m_{q}^{2} Y_{d}^{\dagger} Y_{d}+\frac{4}{5} g_{1}^{2} m_{q}^{2} Y_{u}^{\dagger} Y_{u}-\left|\lambda_{S}\right|^{2} m_{q}^{2} Y_{u}^{\dagger} Y_{u} \\
& -3\left|\lambda_{T}\right|^{2} m_{q}^{2} Y_{u}^{\dagger} Y_{u}+\frac{4}{5} g_{1}^{2} Y_{d}^{\dagger} m_{d}^{2} Y_{d}-2\left|\lambda_{S}\right|^{2} Y_{d}^{\dagger} m_{d}^{2} Y_{d} \\
& -6\left|\lambda_{T}\right|^{2} Y_{d}^{\dagger} m_{d}^{2} Y_{d}+\frac{2}{5} g_{1}^{2} Y_{d}^{\dagger} Y_{d} m_{q}^{2}-\left|\lambda_{S}\right|^{2} Y_{d}^{\dagger} Y_{d} m_{q}^{2} \\
& -3\left|\lambda_{T}\right|^{2} Y_{d}^{\dagger} Y_{d} m_{q}^{2}+\frac{8}{5} g_{1}^{2} Y_{u}^{\dagger} m_{u}^{2} Y_{u}-2\left|\lambda_{S}\right|^{2} Y_{u}^{\dagger} m_{u}^{2} Y_{u} \\
& -6\left|\lambda_{T}\right|^{2} Y_{u}^{\dagger} m_{u}^{2} Y_{u}+\frac{4}{5} g_{1}^{2} Y_{u}^{\dagger} Y_{u} m_{q}^{2}-\left|\lambda_{S}\right|^{2} Y_{u}^{\dagger} Y_{u} m_{q}^{2} \\
& -3\left|\lambda_{T}\right|^{2} Y_{u}^{\dagger} Y_{u} m_{q}^{2}-8 m_{H_{d}}^{2} Y_{d}^{\dagger} Y_{d} Y_{d}^{\dagger} Y_{d}-4 Y_{d}^{\dagger} Y_{d} A_{d}^{\dagger} A_{d}-4 Y_{d}^{\dagger} A_{d} A_{d}^{\dagger} Y_{d} \\
& -8 m_{H_{u}}^{2} Y_{u}^{\dagger} Y_{u} Y_{u}^{\dagger} Y_{u}-4 Y_{u}^{\dagger} Y_{u} A_{u}^{\dagger} A_{u}-4 Y_{u}^{\dagger} A_{u} A_{u}^{\dagger} Y_{u}-4 A_{d}^{\dagger} Y_{d} Y_{d}^{\dagger} A_{d} \\
& -4 A_{d}^{\dagger} A_{d} Y_{d}^{\dagger} Y_{d}-4 A_{u}^{\dagger} Y_{u} Y_{u}^{\dagger} A_{u}-4 A_{u}^{\dagger} A_{u} Y_{u}^{\dagger} Y_{u}-2 m_{q}^{2} Y_{d}^{\dagger} Y_{d} Y_{d}^{\dagger} Y_{d} \\
& -2 m_{q}^{2} Y_{u}^{\dagger} Y_{u} Y_{u}^{\dagger} Y_{u}-4 Y_{d}^{\dagger} m_{d}^{2} Y_{d} Y_{d}^{\dagger} Y_{d}-4 Y_{d}^{\dagger} Y_{d} m_{q}^{2} Y_{d}^{\dagger} Y_{d}-4 Y_{d}^{\dagger} Y_{d} Y_{d}^{\dagger} m_{d}^{2} Y_{d} \\
& -2 Y_{d}^{\dagger} Y_{d} Y_{d}^{\dagger} Y_{d} m_{q}^{2}-4 Y_{u}^{\dagger} m_{u}^{2} Y_{u} Y_{u}^{\dagger} Y_{u}-4 Y_{u}^{\dagger} Y_{u} m_{q}^{2} Y_{u}^{\dagger} Y_{u}-4 Y_{u}^{\dagger} Y_{u} Y_{u}^{\dagger} m_{u}^{2} Y_{u} \\
& -2 Y_{u}^{\dagger} Y_{u} Y_{u}^{\dagger} Y_{u} m_{q}^{2}-2 \lambda_{S}^{*} A_{d}^{\dagger} Y_{d} A_{S}-2 \lambda_{S}^{*} A_{u}^{\dagger} Y_{u} A_{S}-6 \lambda_{T}^{*} A_{d}^{\dagger} Y_{d} A_{T} \\
& -6 \lambda_{T}^{*} A_{u}^{\dagger} Y_{u} A_{T}+6 g_{2}^{4} \mathbf{1} \sigma_{2,2}+\frac{32}{3} g_{3}^{4} \mathbf{1} \sigma_{2,3}+\frac{2}{15} g_{1}^{2} \mathbf{1} \operatorname{Tr} 2 \mathrm{U} 1(1,1)+4 \frac{1}{\sqrt{15}} g_{1} \mathbf{1} \sigma_{3,1} \\
& -12 m_{H_{d}}^{2} Y_{d}^{\dagger} Y_{d} \operatorname{Tr}\left(Y_{d} Y_{d}^{\dagger}\right)-6 A_{d}^{\dagger} A_{d} \operatorname{Tr}\left(Y_{d} Y_{d}^{\dagger}\right)-3 m_{q}^{2} Y_{d}^{\dagger} Y_{d} \operatorname{Tr}\left(Y_{d} Y_{d}^{\dagger}\right) \\
& -6 Y_{d}^{\dagger} m_{d}^{2} Y_{d} \operatorname{Tr}\left(Y_{d} Y_{d}^{\dagger}\right)-3 Y_{d}^{\dagger} Y_{d} m_{q}^{2} \operatorname{Tr}\left(Y_{d} Y_{d}^{\dagger}\right)-4 m_{H_{d}}^{2} Y_{d}^{\dagger} Y_{d} \operatorname{Tr}\left(Y_{e} Y_{e}^{\dagger}\right) \\
& -2 A_{d}^{\dagger} A_{d} \operatorname{Tr}\left(Y_{e} Y_{e}^{\dagger}\right)-m_{q}^{2} Y_{d}^{\dagger} Y_{d} \operatorname{Tr}\left(Y_{e} Y_{e}^{\dagger}\right)-2 Y_{d}^{\dagger} m_{d}^{2} Y_{d} \operatorname{Tr}\left(Y_{e} Y_{e}^{\dagger}\right) \\
& -Y_{d}^{\dagger} Y_{d} m_{q}^{2} \operatorname{Tr}\left(Y_{e} Y_{e}^{\dagger}\right)-12 m_{H_{u}}^{2} Y_{u}^{\dagger} Y_{u} \operatorname{Tr}\left(Y_{u} Y_{u}^{\dagger}\right)-6 A_{u}^{\dagger} A_{u} \operatorname{Tr}\left(Y_{u} Y_{u}^{\dagger}\right) \\
& -3 m_{q}^{2} Y_{u}^{\dagger} Y_{u} \operatorname{Tr}\left(Y_{u} Y_{u}^{\dagger}\right)-6 Y_{u}^{\dagger} m_{u}^{2} Y_{u} \operatorname{Tr}\left(Y_{u} Y_{u}^{\dagger}\right)-3 Y_{u}^{\dagger} Y_{u} m_{q}^{2} \operatorname{Tr}\left(Y_{u} Y_{u}^{\dagger}\right) \\
& -6 A_{d}^{\dagger} Y_{d} \operatorname{Tr}\left(Y_{d}^{\dagger} A_{d}\right)-2 A_{d}^{\dagger} Y_{d} \operatorname{Tr}\left(Y_{e}^{\dagger} A_{e}\right)-6 A_{u}^{\dagger} Y_{u} \operatorname{Tr}\left(Y_{u}^{\dagger} A_{u}\right) \\
& \text { - } 6 Y_{d}^{\dagger} A_{d} \operatorname{Tr}\left(A_{d}^{*} Y_{d}^{T}\right)-6 Y_{d}^{\dagger} Y_{d} \operatorname{Tr}\left(A_{d}^{*} A_{d}^{T}\right)-2 Y_{d}^{\dagger} A_{d} \operatorname{Tr}\left(A_{e}^{*} Y_{e}^{T}\right) \\
& -2 Y_{d}^{\dagger} Y_{d} \operatorname{Tr}\left(A_{e}^{*} A_{e}^{T}\right)-6 Y_{u}^{\dagger} A_{u} \operatorname{Tr}\left(A_{u}^{*} Y_{u}^{T}\right)-6 Y_{u}^{\dagger} Y_{u} \operatorname{Tr}\left(A_{u}^{*} A_{u}^{T}\right) \\
& -6 Y_{d}^{\dagger} Y_{d} \operatorname{Tr}\left(m_{d}^{2} Y_{d} Y_{d}^{\dagger}\right)-2 Y_{d}^{\dagger} Y_{d} \operatorname{Tr}\left(m_{e}^{2} Y_{e} Y_{e}^{\dagger}\right)-2 Y_{d}^{\dagger} Y_{d} \operatorname{Tr}\left(m_{l}^{2} Y_{e}^{\dagger} Y_{e}\right)
\end{aligned}
$$




$$
\begin{aligned}
& -6 Y_{d}^{\dagger} Y_{d} \operatorname{Tr}\left(m_{q}^{2} Y_{d}^{\dagger} Y_{d}\right)-6 Y_{u}^{\dagger} Y_{u} \operatorname{Tr}\left(m_{q}^{2} Y_{u}^{\dagger} Y_{u}\right)-6 Y_{u}^{\dagger} Y_{u} \operatorname{Tr}\left(m_{u}^{2} Y_{u} Y_{u}^{\dagger}\right) \\
& \beta_{m_{l}^{2}}^{(1)}=-\frac{6}{5} g_{1}^{2} \mathbf{1}\left|M_{1}\right|^{2}-6 g_{2}^{2} \mathbf{1}\left|M_{2}\right|^{2}+2 m_{H_{d}}^{2} Y_{e}^{\dagger} Y_{e}+2 A_{e}^{\dagger} A_{e}+m_{l}^{2} Y_{e}^{\dagger} Y_{e}+2 Y_{e}^{\dagger} m_{e}^{2} Y_{e} \\
& +Y_{e}^{\dagger} Y_{e} m_{l}^{2}-\sqrt{\frac{3}{5}} g_{1} \mathbf{1} \sigma_{1,1} \\
& \beta_{m_{l}^{2}}^{(2)}=+\frac{3}{5} g_{2}^{2}\left(115 g_{2}^{2} M_{2}+3 g_{1}^{2}\left(2 M_{2}+M_{1}\right)\right) \mathbf{1} M_{2}^{*}+\frac{12}{5} g_{1}^{2} m_{H_{d}}^{2} Y_{e}^{\dagger} Y_{e}-4 m_{H_{d}}^{2}\left|\lambda_{S}\right|^{2} Y_{e}^{\dagger} Y_{e} \\
& -2 m_{H_{u}}^{2}\left|\lambda_{S}\right|^{2} Y_{e}^{\dagger} Y_{e}-2 m_{S}^{2}\left|\lambda_{S}\right|^{2} Y_{e}^{\dagger} Y_{e}-12 m_{H_{d}}^{2}\left|\lambda_{T}\right|^{2} Y_{e}^{\dagger} Y_{e} \\
& -6 m_{H_{u}}^{2}\left|\lambda_{T}\right|^{2} Y_{e}^{\dagger} Y_{e}-6 m_{T}^{2}\left|\lambda_{T}\right|^{2} Y_{e}^{\dagger} Y_{e}-2\left|A_{S}\right|^{2} Y_{e}^{\dagger} Y_{e}-6\left|A_{T}\right|^{2} Y_{e}^{\dagger} Y_{e} \\
& +\frac{3}{25} g_{1}^{2} M_{1}^{*}\left(-20 Y_{e}^{\dagger} A_{e}+3\left(5 g_{2}^{2}\left(2 M_{1}+M_{2}\right)+69 g_{1}^{2} M_{1}\right) \mathbf{1}+40 M_{1} Y_{e}^{\dagger} Y_{e}\right) \\
& -2 \lambda_{S} A_{S}^{*} Y_{e}^{\dagger} A_{e}-6 \lambda_{T} A_{T}^{*} Y_{e}^{\dagger} A_{e}-\frac{12}{5} g_{1}^{2} M_{1} A_{e}^{\dagger} Y_{e}+\frac{12}{5} g_{1}^{2} A_{e}^{\dagger} A_{e}-2\left|\lambda_{S}\right|^{2} A_{e}^{\dagger} A_{e} \\
& -6\left|\lambda_{T}\right|^{2} A_{e}^{\dagger} A_{e}+\frac{6}{5} g_{1}^{2} m_{l}^{2} Y_{e}^{\dagger} Y_{e}-\left|\lambda_{S}\right|^{2} m_{l}^{2} Y_{e}^{\dagger} Y_{e}-3\left|\lambda_{T}\right|^{2} m_{l}^{2} Y_{e}^{\dagger} Y_{e} \\
& +\frac{12}{5} g_{1}^{2} Y_{e}^{\dagger} m_{e}^{2} Y_{e}-2\left|\lambda_{S}\right|^{2} Y_{e}^{\dagger} m_{e}^{2} Y_{e}-6\left|\lambda_{T}\right|^{2} Y_{e}^{\dagger} m_{e}^{2} Y_{e}+\frac{6}{5} g_{1}^{2} Y_{e}^{\dagger} Y_{e} m_{l}^{2} \\
& -\left|\lambda_{S}\right|^{2} Y_{e}^{\dagger} Y_{e} m_{l}^{2}-3\left|\lambda_{T}\right|^{2} Y_{e}^{\dagger} Y_{e} m_{l}^{2}-8 m_{H_{d}}^{2} Y_{e}^{\dagger} Y_{e} Y_{e}^{\dagger} Y_{e}-4 Y_{e}^{\dagger} Y_{e} A_{e}^{\dagger} A_{e} \\
& -4 Y_{e}^{\dagger} A_{e} A_{e}^{\dagger} Y_{e}-4 A_{e}^{\dagger} Y_{e} Y_{e}^{\dagger} A_{e}-4 A_{e}^{\dagger} A_{e} Y_{e}^{\dagger} Y_{e}-2 m_{l}^{2} Y_{e}^{\dagger} Y_{e} Y_{e}^{\dagger} Y_{e} \\
& -4 Y_{e}^{\dagger} m_{e}^{2} Y_{e} Y_{e}^{\dagger} Y_{e}-4 Y_{e}^{\dagger} Y_{e} m_{l}^{2} Y_{e}^{\dagger} Y_{e}-4 Y_{e}^{\dagger} Y_{e} Y_{e}^{\dagger} m_{e}^{2} Y_{e}-2 Y_{e}^{\dagger} Y_{e} Y_{e}^{\dagger} Y_{e} m_{l}^{2} \\
& -2 \lambda_{S}^{*} A_{e}^{\dagger} Y_{e} A_{S}-6 \lambda_{T}^{*} A_{e}^{\dagger} Y_{e} A_{T}+6 g_{2}^{4} \mathbf{1} \sigma_{2,2}+\frac{6}{5} g_{1}^{2} \mathbf{1} \operatorname{Tr} 2 \mathrm{U} 1(1,1)-4 \sqrt{\frac{3}{5}} g_{1} \mathbf{1} \sigma_{3,1} \\
& -12 m_{H_{d}}^{2} Y_{e}^{\dagger} Y_{e} \operatorname{Tr}\left(Y_{d} Y_{d}^{\dagger}\right)-6 A_{e}^{\dagger} A_{e} \operatorname{Tr}\left(Y_{d} Y_{d}^{\dagger}\right)-3 m_{l}^{2} Y_{e}^{\dagger} Y_{e} \operatorname{Tr}\left(Y_{d} Y_{d}^{\dagger}\right) \\
& -6 Y_{e}^{\dagger} m_{e}^{2} Y_{e} \operatorname{Tr}\left(Y_{d} Y_{d}^{\dagger}\right)-3 Y_{e}^{\dagger} Y_{e} m_{l}^{2} \operatorname{Tr}\left(Y_{d} Y_{d}^{\dagger}\right)-4 m_{H_{d}}^{2} Y_{e}^{\dagger} Y_{e} \operatorname{Tr}\left(Y_{e} Y_{e}^{\dagger}\right) \\
& -2 A_{e}^{\dagger} A_{e} \operatorname{Tr}\left(Y_{e} Y_{e}^{\dagger}\right)-m_{l}^{2} Y_{e}^{\dagger} Y_{e} \operatorname{Tr}\left(Y_{e} Y_{e}^{\dagger}\right)-2 Y_{e}^{\dagger} m_{e}^{2} Y_{e} \operatorname{Tr}\left(Y_{e} Y_{e}^{\dagger}\right) \\
& -Y_{e}^{\dagger} Y_{e} m_{l}^{2} \operatorname{Tr}\left(Y_{e} Y_{e}^{\dagger}\right)-6 A_{e}^{\dagger} Y_{e} \operatorname{Tr}\left(Y_{d}^{\dagger} A_{d}\right)-2 A_{e}^{\dagger} Y_{e} \operatorname{Tr}\left(Y_{e}^{\dagger} A_{e}\right) \\
& -6 Y_{e}^{\dagger} A_{e} \operatorname{Tr}\left(A_{d}^{*} Y_{d}^{T}\right)-6 Y_{e}^{\dagger} Y_{e} \operatorname{Tr}\left(A_{d}^{*} A_{d}^{T}\right)-2 Y_{e}^{\dagger} A_{e} \operatorname{Tr}\left(A_{e}^{*} Y_{e}^{T}\right) \\
& -2 Y_{e}^{\dagger} Y_{e} \operatorname{Tr}\left(A_{e}^{*} A_{e}^{T}\right)-6 Y_{e}^{\dagger} Y_{e} \operatorname{Tr}\left(m_{d}^{2} Y_{d} Y_{d}^{\dagger}\right)-2 Y_{e}^{\dagger} Y_{e} \operatorname{Tr}\left(m_{e}^{2} Y_{e} Y_{e}^{\dagger}\right) \\
& -2 Y_{e}^{\dagger} Y_{e} \operatorname{Tr}\left(m_{l}^{2} Y_{e}^{\dagger} Y_{e}\right)-6 Y_{e}^{\dagger} Y_{e} \operatorname{Tr}\left(m_{q}^{2} Y_{d}^{\dagger} Y_{d}\right) \\
& \beta_{m_{H_{d}}^{2}}^{(1)}=-\frac{6}{5} g_{1}^{2}\left|M_{1}\right|^{2}-6 g_{2}^{2}\left|M_{2}\right|^{2}+2 m_{H_{d}}^{2}\left|\lambda_{S}\right|^{2}+2 m_{H_{u}}^{2}\left|\lambda_{S}\right|^{2} \\
& +2 m_{S}^{2}\left|\lambda_{S}\right|^{2}+6 m_{H_{d}}^{2}\left|\lambda_{T}\right|^{2}+6 m_{H_{u}}^{2}\left|\lambda_{T}\right|^{2} \\
& +6 m_{T}^{2}\left|\lambda_{T}\right|^{2}+2\left|A_{S}\right|^{2}+6\left|A_{T}\right|^{2}-\sqrt{\frac{3}{5}} g_{1} \sigma_{1,1}+6 m_{H_{d}}^{2} \operatorname{Tr}\left(Y_{d} Y_{d}^{\dagger}\right) \\
& +2 m_{H_{d}}^{2} \operatorname{Tr}\left(Y_{e} Y_{e}^{\dagger}\right)+6 \operatorname{Tr}\left(A_{d}^{*} A_{d}^{T}\right) \\
& +2 \operatorname{Tr}\left(A_{e}^{*} A_{e}^{T}\right)+6 \operatorname{Tr}\left(m_{d}^{2} Y_{d} Y_{d}^{\dagger}\right)+2 \operatorname{Tr}\left(m_{e}^{2} Y_{e} Y_{e}^{\dagger}\right) \\
& +2 \operatorname{Tr}\left(m_{l}^{2} Y_{e}^{\dagger} Y_{e}\right)+6 \operatorname{Tr}\left(m_{q}^{2} Y_{d}^{\dagger} Y_{d}\right)
\end{aligned}
$$




$$
\begin{aligned}
& \beta_{m_{H_{d}}^{2}}^{(2)}=\frac{1}{25}\left(g _ { 1 } ^ { 2 } M _ { 1 } ^ { * } \left(621 g_{1}^{2} M_{1}+90 g_{2}^{2} M_{1}+45 g_{2}^{2} M_{2}-40 M_{1} \operatorname{Tr}\left(Y_{d} Y_{d}^{\dagger}\right)+120 M_{1} \operatorname{Tr}\left(Y_{e} Y_{e}^{\dagger}\right)\right.\right. \\
& \left.+20 \operatorname{Tr}\left(Y_{d}^{\dagger} A_{d}\right)-60 \operatorname{Tr}\left(Y_{e}^{\dagger} A_{e}\right)\right) \\
& +5\left(3 g_{2}^{2} M_{2}^{*}\left(115 g_{2}^{2} M_{2}+3 g_{1}^{2}\left(2 M_{2}+M_{1}\right)+40 \lambda_{T}^{*}\left(2 M_{2} \lambda_{T}-A_{T}\right)\right)\right. \\
& -2\left(-60 g_{2}^{2} m_{H_{d}}^{2}\left|\lambda_{T}\right|^{2}-60 g_{2}^{2} m_{H_{u}}^{2}\left|\lambda_{T}\right|^{2}-60 g_{2}^{2} m_{T}^{2}\left|\lambda_{T}\right|^{2}-60 g_{2}^{2}\left|A_{T}\right|^{2}\right. \\
& +30\left(m_{H_{d}}^{2}+m_{H_{u}}^{2}+m_{S}^{2}\right) \lambda_{S}^{2} \lambda_{S}^{*, 2}+150 m_{H_{d}}^{2} \lambda_{T}^{2} \lambda_{T}^{*, 2}+150 m_{H_{u}}^{2} \lambda_{T}^{2} \lambda_{T}^{*, 2}+150 m_{T}^{2} \lambda_{T}^{2} \lambda_{T}^{*, 2} \\
& +60 g_{2}^{2} M_{2} \lambda_{T} A_{T}^{*}+30\left|\lambda_{T}\right|^{2} A_{S}^{*} A_{S}+10 \kappa_{S}^{*}\left(\left(4 m_{S}^{2}+m_{H_{d}}^{2}+m_{H_{u}}^{2}\right) \kappa_{S}\left|\lambda_{S}\right|^{2}+A_{S}^{*}\left(\kappa_{S} A_{S}+\lambda_{S} A_{\kappa}\right)\right) \\
& +30 \lambda_{S} \lambda_{T}^{*} A_{S}^{*} A_{T}+300\left|\lambda_{T}\right|^{2} A_{T}^{*} A_{T}-15 g_{2}^{4} \sigma_{2,2}-3 g_{1}^{2} \operatorname{Tr} 2 \mathrm{U} 1(1,1)+2 \sqrt{15} g_{1} \sigma_{3,1} \\
& +2 g_{1}^{2} m_{H_{d}}^{2} \operatorname{Tr}\left(Y_{d} Y_{d}^{\dagger}\right)-80 g_{3}^{2} m_{H_{d}}^{2} \operatorname{Tr}\left(Y_{d} Y_{d}^{\dagger}\right)-160 g_{3}^{2}\left|M_{3}\right|^{2} \operatorname{Tr}\left(Y_{d} Y_{d}^{\dagger}\right) \\
& -6 g_{1}^{2} m_{H_{d}}^{2} \operatorname{Tr}\left(Y_{e} Y_{e}^{\dagger}\right)+45 m_{H_{d}}^{2}\left|\lambda_{T}\right|^{2} \operatorname{Tr}\left(Y_{u} Y_{u}^{\dagger}\right)+90 m_{H_{u}}^{2}\left|\lambda_{T}\right|^{2} \operatorname{Tr}\left(Y_{u} Y_{u}^{\dagger}\right) \\
& +45 m_{T}^{2}\left|\lambda_{T}\right|^{2} \operatorname{Tr}\left(Y_{u} Y_{u}^{\dagger}\right)+15\left|A_{S}\right|^{2} \operatorname{Tr}\left(Y_{u} Y_{u}^{\dagger}\right)+45\left|A_{T}\right|^{2} \operatorname{Tr}\left(Y_{u} Y_{u}^{\dagger}\right)+80 g_{3}^{2} M_{3}^{*} \operatorname{Tr}\left(Y_{d}^{\dagger} A_{d}\right) \\
& +15 \lambda_{S} A_{S}^{*} \operatorname{Tr}\left(Y_{u}^{\dagger} A_{u}\right)+45 \lambda_{T} A_{T}^{*} \operatorname{Tr}\left(Y_{u}^{\dagger} A_{u}\right)-2 g_{1}^{2} M_{1} \operatorname{Tr}\left(A_{d}^{*} Y_{d}^{T}\right)+80 g_{3}^{2} M_{3} \operatorname{Tr}\left(A_{d}^{*} Y_{d}^{T}\right) \\
& +2 g_{1}^{2} \operatorname{Tr}\left(A_{d}^{*} A_{d}^{T}\right)-80 g_{3}^{2} \operatorname{Tr}\left(A_{d}^{*} A_{d}^{T}\right)+6 g_{1}^{2} M_{1} \operatorname{Tr}\left(A_{e}^{*} Y_{e}^{T}\right)-6 g_{1}^{2} \operatorname{Tr}\left(A_{e}^{*} A_{e}^{T}\right) \\
& +45 \lambda_{T}^{*} A_{T} \operatorname{Tr}\left(A_{u}^{*} Y_{u}^{T}\right)+45\left|\lambda_{T}\right|^{2} \operatorname{Tr}\left(A_{u}^{*} A_{u}^{T}\right)+2 g_{1}^{2} \operatorname{Tr}\left(m_{d}^{2} Y_{d} Y_{d}^{\dagger}\right)-80 g_{3}^{2} \operatorname{Tr}\left(m_{d}^{2} Y_{d} Y_{d}^{\dagger}\right) \\
& -6 g_{1}^{2} \operatorname{Tr}\left(m_{e}^{2} Y_{e} Y_{e}^{\dagger}\right)-6 g_{1}^{2} \operatorname{Tr}\left(m_{l}^{2} Y_{e}^{\dagger} Y_{e}\right)+2 g_{1}^{2} \operatorname{Tr}\left(m_{q}^{2} Y_{d}^{\dagger} Y_{d}\right)-80 g_{3}^{2} \operatorname{Tr}\left(m_{q}^{2} Y_{d}^{\dagger} Y_{d}\right) \\
& +45\left|\lambda_{T}\right|^{2} \operatorname{Tr}\left(m_{q}^{2} Y_{u}^{\dagger} Y_{u}\right)+45\left|\lambda_{T}\right|^{2} \operatorname{Tr}\left(m_{u}^{2} Y_{u} Y_{u}^{\dagger}\right) \\
& +5 \lambda_{S}^{*}\left(6\left(2 m_{H_{d}}^{2}+2 m_{H_{u}}^{2}+m_{S}^{2}+m_{T}^{2}\right) \lambda_{S}\left|\lambda_{T}\right|^{2}+2 A_{\kappa}^{*}\left(\kappa_{S} A_{S}+\lambda_{S} A_{\kappa}\right)\right. \\
& +3\left(4 \lambda_{S}\left|A_{S}\right|^{2}+2 A_{T}^{*}\left(\lambda_{S} A_{T}+\lambda_{T} A_{S}\right)+m_{H_{d}}^{2} \lambda_{S} \operatorname{Tr}\left(Y_{u} Y_{u}^{\dagger}\right)+2 m_{H_{u}}^{2} \lambda_{S} \operatorname{Tr}\left(Y_{u} Y_{u}^{\dagger}\right)\right. \\
& +m_{S}^{2} \lambda_{S} \operatorname{Tr}\left(Y_{u} Y_{u}^{\dagger}\right) \\
& \left.\left.+A_{S} \operatorname{Tr}\left(A_{u}^{*} Y_{u}^{T}\right)+\lambda_{S} \operatorname{Tr}\left(A_{u}^{*} A_{u}^{T}\right)+\lambda_{S} \operatorname{Tr}\left(m_{q}^{2} Y_{u}^{\dagger} Y_{u}\right)+\lambda_{S} \operatorname{Tr}\left(m_{u}^{2} Y_{u} Y_{u}^{\dagger}\right)\right)\right) \\
& +90 m_{H_{d}}^{2} \operatorname{Tr}\left(Y_{d} Y_{d}^{\dagger} Y_{d} Y_{d}^{\dagger}\right)+90 \operatorname{Tr}\left(Y_{d} Y_{d}^{\dagger} A_{d} A_{d}^{\dagger}\right)+15 m_{H_{d}}^{2} \operatorname{Tr}\left(Y_{d} Y_{u}^{\dagger} Y_{u} Y_{d}^{\dagger}\right) \\
& +15 m_{H_{u}}^{2} \operatorname{Tr}\left(Y_{d} Y_{u}^{\dagger} Y_{u} Y_{d}^{\dagger}\right)+15 \operatorname{Tr}\left(Y_{d} Y_{u}^{\dagger} A_{u} A_{d}^{\dagger}\right)+90 \operatorname{Tr}\left(Y_{d} A_{d}^{\dagger} A_{d} Y_{d}^{\dagger}\right) \\
& +15 \operatorname{Tr}\left(Y_{d} A_{u}^{\dagger} A_{u} Y_{d}^{\dagger}\right)+30 m_{H_{d}}^{2} \operatorname{Tr}\left(Y_{e} Y_{e}^{\dagger} Y_{e} Y_{e}^{\dagger}\right)+30 \operatorname{Tr}\left(Y_{e} Y_{e}^{\dagger} A_{e} A_{e}^{\dagger}\right)+30 \operatorname{Tr}\left(Y_{e} A_{e}^{\dagger} A_{e} Y_{e}^{\dagger}\right) \\
& +15 \operatorname{Tr}\left(Y_{u} Y_{d}^{\dagger} A_{d} A_{u}^{\dagger}\right)+15 \operatorname{Tr}\left(Y_{u} A_{d}^{\dagger} A_{d} Y_{u}^{\dagger}\right)+90 \operatorname{Tr}\left(m_{d}^{2} Y_{d} Y_{d}^{\dagger} Y_{d} Y_{d}^{\dagger}\right)+15 \operatorname{Tr}\left(m_{d}^{2} Y_{d} Y_{u}^{\dagger} Y_{u} Y_{d}^{\dagger}\right) \\
& +30 \operatorname{Tr}\left(m_{e}^{2} Y_{e} Y_{e}^{\dagger} Y_{e} Y_{e}^{\dagger}\right)+30 \operatorname{Tr}\left(m_{l}^{2} Y_{e}^{\dagger} Y_{e} Y_{e}^{\dagger} Y_{e}\right)+90 \operatorname{Tr}\left(m_{q}^{2} Y_{d}^{\dagger} Y_{d} Y_{d}^{\dagger} Y_{d}\right)+15 \operatorname{Tr}\left(m_{q}^{2} Y_{d}^{\dagger} Y_{d} Y_{u}^{\dagger} Y_{u}\right) \\
& \left.\left.\left.+15 \operatorname{Tr}\left(m_{q}^{2} Y_{u}^{\dagger} Y_{u} Y_{d}^{\dagger} Y_{d}\right)+15 \operatorname{Tr}\left(m_{u}^{2} Y_{u} Y_{d}^{\dagger} Y_{d} Y_{u}^{\dagger}\right)\right)\right)\right) \\
& \beta_{m_{H_{u}}^{2}}^{(1)}=-\frac{6}{5} g_{1}^{2}\left|M_{1}\right|^{2}-6 g_{2}^{2}\left|M_{2}\right|^{2}+2 m_{H_{d}}^{2}\left|\lambda_{S}\right|^{2}+2 m_{H_{u}}^{2}\left|\lambda_{S}\right|^{2}+2 m_{S}^{2}\left|\lambda_{S}\right|^{2}+6 m_{H_{d}}^{2}\left|\lambda_{T}\right|^{2}+6 m_{H_{u}}^{2}\left|\lambda_{T}\right|^{2} \\
& +6 m_{T}^{2}\left|\lambda_{T}\right|^{2}+2\left|A_{S}\right|^{2}+6\left|A_{T}\right|^{2}+\sqrt{\frac{3}{5}} g_{1} \sigma_{1,1}+6 m_{H_{u}}^{2} \operatorname{Tr}\left(Y_{u} Y_{u}^{\dagger}\right)+6 \operatorname{Tr}\left(A_{u}^{*} A_{u}^{T}\right)+6 \operatorname{Tr}\left(m_{q}^{2} Y_{u}^{\dagger} Y_{u}\right) \\
& +6 \operatorname{Tr}\left(m_{u}^{2} Y_{u} Y_{u}^{\dagger}\right)
\end{aligned}
$$




$$
\begin{aligned}
& \beta_{m_{H_{u}}^{2}}^{(2)}=\frac{1}{25}\left(g_{1}^{2} M_{1}^{*}\left(-40 \operatorname{Tr}\left(Y_{u}^{\dagger} A_{u}\right)+45 g_{2}^{2} M_{2}+621 g_{1}^{2} M_{1}+80 M_{1} \operatorname{Tr}\left(Y_{u} Y_{u}^{\dagger}\right)+90 g_{2}^{2} M_{1}\right)\right. \\
& +5\left(3 g_{2}^{2} M_{2}^{*}\left(115 g_{2}^{2} M_{2}+3 g_{1}^{2}\left(2 M_{2}+M_{1}\right)+40 \lambda_{T}^{*}\left(2 M_{2} \lambda_{T}-A_{T}\right)\right)\right. \\
& -2\left(-60 g_{2}^{2} m_{H_{d}}^{2}\left|\lambda_{T}\right|^{2}-60 g_{2}^{2} m_{H_{u}}^{2}\left|\lambda_{T}\right|^{2}-60 g_{2}^{2} m_{T}^{2}\left|\lambda_{T}\right|^{2}-60 g_{2}^{2}\left|A_{T}\right|^{2}\right. \\
& +30\left(m_{H_{d}}^{2}+m_{H_{u}}^{2}+m_{S}^{2}\right) \lambda_{S}^{2} \lambda_{S}^{*, 2}+150 m_{H_{d}}^{2} \lambda_{T}^{2} \lambda_{T}^{*, 2}+150 m_{H_{u}}^{2} \lambda_{T}^{2} \lambda_{T}^{*, 2}+150 m_{T}^{2} \lambda_{T}^{2} \lambda_{T}^{*, 2} \\
& +60 g_{2}^{2} M_{2} \lambda_{T} A_{T}^{*}+30\left|\lambda_{T}\right|^{2} A_{S}^{*} A_{S}+10 \kappa_{S}^{*}\left(\left(4 m_{S}^{2}+m_{H_{d}}^{2}+m_{H_{u}}^{2}\right) \kappa_{S}\left|\lambda_{S}\right|^{2}+A_{S}^{*}\left(\kappa_{S} A_{S}+\lambda_{S} A_{\kappa}\right)\right) \\
& +30 \lambda_{S} \lambda_{T}^{*} A_{S}^{*} A_{T}+300\left|\lambda_{T}\right|^{2} A_{T}^{*} A_{T}-15 g_{2}^{4} \sigma_{2,2}-3 g_{1}^{2} \operatorname{Tr} 2 \mathrm{U} 1(1,1)-2 \sqrt{15} g_{1} \sigma_{3,1} \\
& +90 m_{H_{d}}^{2}\left|\lambda_{T}\right|^{2} \operatorname{Tr}\left(Y_{d} Y_{d}^{\dagger}\right)+45 m_{H_{u}}^{2}\left|\lambda_{T}\right|^{2} \operatorname{Tr}\left(Y_{d} Y_{d}^{\dagger}\right)+45 m_{T}^{2}\left|\lambda_{T}\right|^{2} \operatorname{Tr}\left(Y_{d} Y_{d}^{\dagger}\right) \\
& +15\left|A_{S}\right|^{2} \operatorname{Tr}\left(Y_{d} Y_{d}^{\dagger}\right)+45\left|A_{T}\right|^{2} \operatorname{Tr}\left(Y_{d} Y_{d}^{\dagger}\right)+30 m_{H_{d}}^{2}\left|\lambda_{T}\right|^{2} \operatorname{Tr}\left(Y_{e} Y_{e}^{\dagger}\right)+15 m_{H_{u}}^{2}\left|\lambda_{T}\right|^{2} \operatorname{Tr}\left(Y_{e} Y_{e}^{\dagger}\right) \\
& +15 m_{T}^{2}\left|\lambda_{T}\right|^{2} \operatorname{Tr}\left(Y_{e} Y_{e}^{\dagger}\right)+5\left|A_{S}\right|^{2} \operatorname{Tr}\left(Y_{e} Y_{e}^{\dagger}\right)+15\left|A_{T}\right|^{2} \operatorname{Tr}\left(Y_{e} Y_{e}^{\dagger}\right)-4 g_{1}^{2} m_{H_{u}}^{2} \operatorname{Tr}\left(Y_{u} Y_{u}^{\dagger}\right) \\
& -80 g_{3}^{2} m_{H_{u}}^{2} \operatorname{Tr}\left(Y_{u} Y_{u}^{\dagger}\right)-160 g_{3}^{2}\left|M_{3}\right|^{2} \operatorname{Tr}\left(Y_{u} Y_{u}^{\dagger}\right)+15 \lambda_{S} A_{S}^{*} \operatorname{Tr}\left(Y_{d}^{\dagger} A_{d}\right)+45 \lambda_{T} A_{T}^{*} \operatorname{Tr}\left(Y_{d}^{\dagger} A_{d}\right) \\
& +5 \lambda_{S} A_{S}^{*} \operatorname{Tr}\left(Y_{e}^{\dagger} A_{e}\right)+15 \lambda_{T} A_{T}^{*} \operatorname{Tr}\left(Y_{e}^{\dagger} A_{e}\right)+80 g_{3}^{2} M_{3}^{*} \operatorname{Tr}\left(Y_{u}^{\dagger} A_{u}\right)+45 \lambda_{T}^{*} A_{T} \operatorname{Tr}\left(A_{d}^{*} Y_{d}^{T}\right) \\
& +45\left|\lambda_{T}\right|^{2} \operatorname{Tr}\left(A_{d}^{*} A_{d}^{T}\right)+15 \lambda_{T}^{*} A_{T} \operatorname{Tr}\left(A_{e}^{*} Y_{e}^{T}\right)+15\left|\lambda_{T}\right|^{2} \operatorname{Tr}\left(A_{e}^{*} A_{e}^{T}\right)+4 g_{1}^{2} M_{1} \operatorname{Tr}\left(A_{u}^{*} Y_{u}^{T}\right) \\
& +80 g_{3}^{2} M_{3} \operatorname{Tr}\left(A_{u}^{*} Y_{u}^{T}\right)-4 g_{1}^{2} \operatorname{Tr}\left(A_{u}^{*} A_{u}^{T}\right)-80 g_{3}^{2} \operatorname{Tr}\left(A_{u}^{*} A_{u}^{T}\right)+45\left|\lambda_{T}\right|^{2} \operatorname{Tr}\left(m_{d}^{2} Y_{d} Y_{d}^{\dagger}\right) \\
& +15\left|\lambda_{T}\right|^{2} \operatorname{Tr}\left(m_{e}^{2} Y_{e} Y_{e}^{\dagger}\right)+15\left|\lambda_{T}\right|^{2} \operatorname{Tr}\left(m_{l}^{2} Y_{e}^{\dagger} Y_{e}\right)+45\left|\lambda_{T}\right|^{2} \operatorname{Tr}\left(m_{q}^{2} Y_{d}^{\dagger} Y_{d}\right) \\
& +5 \lambda_{S}^{*}\left(6\left(2 m_{H_{d}}^{2}+2 m_{H_{u}}^{2}+m_{S}^{2}+m_{T}^{2}\right) \lambda_{S}\left|\lambda_{T}\right|^{2}+12 \lambda_{S}\left|A_{S}\right|^{2}\right. \\
& +6 \lambda_{S}\left|A_{T}\right|^{2}+6 \lambda_{T} A_{T}^{*} A_{S}+2 A_{\kappa}^{*}\left(\kappa_{S} A_{S}+\lambda_{S} A_{\kappa}\right) \\
& +6 m_{H_{d}}^{2} \lambda_{S} \operatorname{Tr}\left(Y_{d} Y_{d}^{\dagger}\right)+3 m_{H_{u}}^{2} \lambda_{S} \operatorname{Tr}\left(Y_{d} Y_{d}^{\dagger}\right)+3 m_{S}^{2} \lambda_{S} \operatorname{Tr}\left(Y_{d} Y_{d}^{\dagger}\right)+2 m_{H_{d}}^{2} \lambda_{S} \operatorname{Tr}\left(Y_{e} Y_{e}^{\dagger}\right) \\
& +m_{H_{u}}^{2} \lambda_{S} \operatorname{Tr}\left(Y_{e} Y_{e}^{\dagger}\right)+m_{S}^{2} \lambda_{S} \operatorname{Tr}\left(Y_{e} Y_{e}^{\dagger}\right)+3 A_{S} \operatorname{Tr}\left(A_{d}^{*} Y_{d}^{T}\right)+3 \lambda_{S} \operatorname{Tr}\left(A_{d}^{*} A_{d}^{T}\right)+A_{S} \operatorname{Tr}\left(A_{e}^{*} Y_{e}^{T}\right) \\
& +\lambda_{S} \operatorname{Tr}\left(A_{e}^{*} A_{e}^{T}\right)+3 \lambda_{S} \operatorname{Tr}\left(m_{d}^{2} Y_{d} Y_{d}^{\dagger}\right)+\lambda_{S} \operatorname{Tr}\left(m_{e}^{2} Y_{e} Y_{e}^{\dagger}\right)+\lambda_{S} \operatorname{Tr}\left(m_{l}^{2} Y_{e}^{\dagger} Y_{e}\right) \\
& \left.+3 \lambda_{S} \operatorname{Tr}\left(m_{q}^{2} Y_{d}^{\dagger} Y_{d}\right)\right) \\
& -4 g_{1}^{2} \operatorname{Tr}\left(m_{q}^{2} Y_{u}^{\dagger} Y_{u}\right)-80 g_{3}^{2} \operatorname{Tr}\left(m_{q}^{2} Y_{u}^{\dagger} Y_{u}\right)-4 g_{1}^{2} \operatorname{Tr}\left(m_{u}^{2} Y_{u} Y_{u}^{\dagger}\right)-80 g_{3}^{2} \operatorname{Tr}\left(m_{u}^{2} Y_{u} Y_{u}^{\dagger}\right) \\
& +15 m_{H_{d}}^{2} \operatorname{Tr}\left(Y_{d} Y_{u}^{\dagger} Y_{u} Y_{d}^{\dagger}\right)+15 m_{H_{u}}^{2} \operatorname{Tr}\left(Y_{d} Y_{u}^{\dagger} Y_{u} Y_{d}^{\dagger}\right)+15 \operatorname{Tr}\left(Y_{d} Y_{u}^{\dagger} A_{u} A_{d}^{\dagger}\right) \\
& +15 \operatorname{Tr}\left(Y_{d} A_{u}^{\dagger} A_{u} Y_{d}^{\dagger}\right)+15 \operatorname{Tr}\left(Y_{u} Y_{d}^{\dagger} A_{d} A_{u}^{\dagger}\right)+90 m_{H_{u}}^{2} \operatorname{Tr}\left(Y_{u} Y_{u}^{\dagger} Y_{u} Y_{u}^{\dagger}\right)+90 \operatorname{Tr}\left(Y_{u} Y_{u}^{\dagger} A_{u} A_{u}^{\dagger}\right) \\
& +15 \operatorname{Tr}\left(Y_{u} A_{d}^{\dagger} A_{d} Y_{u}^{\dagger}\right)+90 \operatorname{Tr}\left(Y_{u} A_{u}^{\dagger} A_{u} Y_{u}^{\dagger}\right)+15 \operatorname{Tr}\left(m_{d}^{2} Y_{d} Y_{u}^{\dagger} Y_{u} Y_{d}^{\dagger}\right) \\
& +15 \operatorname{Tr}\left(m_{q}^{2} Y_{d}^{\dagger} Y_{d} Y_{u}^{\dagger} Y_{u}\right)+15 \operatorname{Tr}\left(m_{q}^{2} Y_{u}^{\dagger} Y_{u} Y_{d}^{\dagger} Y_{d}\right)+90 \operatorname{Tr}\left(m_{q}^{2} Y_{u}^{\dagger} Y_{u} Y_{u}^{\dagger} Y_{u}\right) \\
& \left.\left.\left.+15 \operatorname{Tr}\left(m_{u}^{2} Y_{u} Y_{d}^{\dagger} Y_{d} Y_{u}^{\dagger}\right)+90 \operatorname{Tr}\left(m_{u}^{2} Y_{u} Y_{u}^{\dagger} Y_{u} Y_{u}^{\dagger}\right)\right)\right)\right) \\
& \beta_{m_{d}^{2}}^{(1)}=-\frac{8}{15} g_{1}^{2} \mathbf{1}\left|M_{1}\right|^{2}-\frac{32}{3} g_{3}^{2} \mathbf{1}\left|M_{3}\right|^{2}+4 m_{H_{d}}^{2} Y_{d} Y_{d}^{\dagger}+4 A_{d} A_{d}^{\dagger}+2 m_{d}^{2} Y_{d} Y_{d}^{\dagger}+4 Y_{d} m_{q}^{2} Y_{d}^{\dagger} \\
& +2 Y_{d} Y_{d}^{\dagger} m_{d}^{2}+2 \frac{1}{\sqrt{15}} g_{1} \mathbf{1} \sigma_{1,1}
\end{aligned}
$$




$$
\begin{aligned}
& \beta_{m_{d}^{2}}^{(2)}=+\frac{32}{45} g_{3}^{2}\left(2 g_{1}^{2}\left(2 M_{3}+M_{1}\right)+75 g_{3}^{2} M_{3}\right) \mathbf{1} M_{3}^{*}+\frac{4}{5} g_{1}^{2} m_{H_{d}}^{2} Y_{d} Y_{d}^{\dagger}+12 g_{2}^{2} m_{H_{d}}^{2} Y_{d} Y_{d}^{\dagger} \\
& +24 g_{2}^{2}\left|M_{2}\right|^{2} Y_{d} Y_{d}^{\dagger}-8 m_{H_{d}}^{2}\left|\lambda_{S}\right|^{2} Y_{d} Y_{d}^{\dagger}-4 m_{H_{u}}^{2}\left|\lambda_{S}\right|^{2} Y_{d} Y_{d}^{\dagger} \\
& -4 m_{S}^{2}\left|\lambda_{S}\right|^{2} Y_{d} Y_{d}^{\dagger}-24 m_{H_{d}}^{2}\left|\lambda_{T}\right|^{2} Y_{d} Y_{d}^{\dagger}-12 m_{H_{u}}^{2}\left|\lambda_{T}\right|^{2} Y_{d} Y_{d}^{\dagger} \\
& -12 m_{T}^{2}\left|\lambda_{T}\right|^{2} Y_{d} Y_{d}^{\dagger}-4\left|A_{S}\right|^{2} Y_{d} Y_{d}^{\dagger}-12\left|A_{T}\right|^{2} Y_{d} Y_{d}^{\dagger}-\frac{4}{5} g_{1}^{2} M_{1} Y_{d} A_{d}^{\dagger} \\
& -12 g_{2}^{2} M_{2} Y_{d} A_{d}^{\dagger}+\frac{4}{225} g_{1}^{2} M_{1}^{*}\left(2\left(303 g_{1}^{2} M_{1}+40 g_{3}^{2}\left(2 M_{1}+M_{3}\right)\right) \mathbf{1}-45 A_{d} Y_{d}^{\dagger}+90 M_{1} Y_{d} Y_{d}^{\dagger}\right) \\
& -12 g_{2}^{2} M_{2}^{*} A_{d} Y_{d}^{\dagger}-4 \lambda_{S} A_{S}^{*} A_{d} Y_{d}^{\dagger}-12 \lambda_{T} A_{T}^{*} A_{d} Y_{d}^{\dagger}+\frac{4}{5} g_{1}^{2} A_{d} A_{d}^{\dagger} \\
& +12 g_{2}^{2} A_{d} A_{d}^{\dagger}-4\left|\lambda_{S}\right|^{2} A_{d} A_{d}^{\dagger}-12\left|\lambda_{T}\right|^{2} A_{d} A_{d}^{\dagger}+\frac{2}{5} g_{1}^{2} m_{d}^{2} Y_{d} Y_{d}^{\dagger} \\
& +6 g_{2}^{2} m_{d}^{2} Y_{d} Y_{d}^{\dagger}-2\left|\lambda_{S}\right|^{2} m_{d}^{2} Y_{d} Y_{d}^{\dagger}-6\left|\lambda_{T}\right|^{2} m_{d}^{2} Y_{d} Y_{d}^{\dagger}+\frac{4}{5} g_{1}^{2} Y_{d} m_{q}^{2} Y_{d}^{\dagger} \\
& +12 g_{2}^{2} Y_{d} m_{q}^{2} Y_{d}^{\dagger}-4\left|\lambda_{S}\right|^{2} Y_{d} m_{q}^{2} Y_{d}^{\dagger}-12\left|\lambda_{T}\right|^{2} Y_{d} m_{q}^{2} Y_{d}^{\dagger}+\frac{2}{5} g_{1}^{2} Y_{d} Y_{d}^{\dagger} m_{d}^{2} \\
& +6 g_{2}^{2} Y_{d} Y_{d}^{\dagger} m_{d}^{2}-2\left|\lambda_{S}\right|^{2} Y_{d} Y_{d}^{\dagger} m_{d}^{2}-6\left|\lambda_{T}\right|^{2} Y_{d} Y_{d}^{\dagger} m_{d}^{2}-8 m_{H_{d}}^{2} Y_{d} Y_{d}^{\dagger} Y_{d} Y_{d}^{\dagger} \\
& -4 Y_{d} Y_{d}^{\dagger} A_{d} A_{d}^{\dagger}-4 m_{H_{d}}^{2} Y_{d} Y_{u}^{\dagger} Y_{u} Y_{d}^{\dagger}-4 m_{H_{u}}^{2} Y_{d} Y_{u}^{\dagger} Y_{u} Y_{d}^{\dagger} \\
& -4 Y_{d} Y_{u}^{\dagger} A_{u} A_{d}^{\dagger}-4 Y_{d} A_{d}^{\dagger} A_{d} Y_{d}^{\dagger}-4 Y_{d} A_{u}^{\dagger} A_{u} Y_{d}^{\dagger}-4 A_{d} Y_{d}^{\dagger} Y_{d} A_{d}^{\dagger} \\
& -4 A_{d} Y_{u}^{\dagger} Y_{u} A_{d}^{\dagger}-4 A_{d} A_{d}^{\dagger} Y_{d} Y_{d}^{\dagger}-4 A_{d} A_{u}^{\dagger} Y_{u} Y_{d}^{\dagger}-2 m_{d}^{2} Y_{d} Y_{d}^{\dagger} Y_{d} Y_{d}^{\dagger} \\
& -2 m_{d}^{2} Y_{d} Y_{u}^{\dagger} Y_{u} Y_{d}^{\dagger}-4 Y_{d} m_{q}^{2} Y_{d}^{\dagger} Y_{d} Y_{d}^{\dagger}-4 Y_{d} m_{q}^{2} Y_{u}^{\dagger} Y_{u} Y_{d}^{\dagger}-4 Y_{d} Y_{d}^{\dagger} m_{d}^{2} Y_{d} Y_{d}^{\dagger} \\
& -4 Y_{d} Y_{d}^{\dagger} Y_{d} m_{q}^{2} Y_{d}^{\dagger}-2 Y_{d} Y_{d}^{\dagger} Y_{d} Y_{d}^{\dagger} m_{d}^{2}-4 Y_{d} Y_{u}^{\dagger} m_{u}^{2} Y_{u} Y_{d}^{\dagger}-4 Y_{d} Y_{u}^{\dagger} Y_{u} m_{q}^{2} Y_{d}^{\dagger} \\
& -2 Y_{d} Y_{u}^{\dagger} Y_{u} Y_{d}^{\dagger} m_{d}^{2}-4 \lambda_{S}^{*} Y_{d} A_{d}^{\dagger} A_{S}-12 \lambda_{T}^{*} Y_{d} A_{d}^{\dagger} A_{T}+\frac{32}{3} g_{3}^{4} 1 \sigma_{2,3}+\frac{8}{15} g_{1}^{2} 1 \operatorname{Tr} 2 \mathrm{U} 1(1,1) \\
& +8 \frac{1}{\sqrt{15}} g_{1} \mathbf{1} \sigma_{3,1}-24 m_{H_{d}}^{2} Y_{d} Y_{d}^{\dagger} \operatorname{Tr}\left(Y_{d} Y_{d}^{\dagger}\right)-12 A_{d} A_{d}^{\dagger} \operatorname{Tr}\left(Y_{d} Y_{d}^{\dagger}\right) \\
& -6 m_{d}^{2} Y_{d} Y_{d}^{\dagger} \operatorname{Tr}\left(Y_{d} Y_{d}^{\dagger}\right)-12 Y_{d} m_{q}^{2} Y_{d}^{\dagger} \operatorname{Tr}\left(Y_{d} Y_{d}^{\dagger}\right)-6 Y_{d} Y_{d}^{\dagger} m_{d}^{2} \operatorname{Tr}\left(Y_{d} Y_{d}^{\dagger}\right) \\
& -8 m_{H_{d}}^{2} Y_{d} Y_{d}^{\dagger} \operatorname{Tr}\left(Y_{e} Y_{e}^{\dagger}\right)-4 A_{d} A_{d}^{\dagger} \operatorname{Tr}\left(Y_{e} Y_{e}^{\dagger}\right)-2 m_{d}^{2} Y_{d} Y_{d}^{\dagger} \operatorname{Tr}\left(Y_{e} Y_{e}^{\dagger}\right) \\
& -4 Y_{d} m_{q}^{2} Y_{d}^{\dagger} \operatorname{Tr}\left(Y_{e} Y_{e}^{\dagger}\right)-2 Y_{d} Y_{d}^{\dagger} m_{d}^{2} \operatorname{Tr}\left(Y_{e} Y_{e}^{\dagger}\right)-12 Y_{d} A_{d}^{\dagger} \operatorname{Tr}\left(Y_{d}^{\dagger} A_{d}\right) \\
& -4 Y_{d} A_{d}^{\dagger} \operatorname{Tr}\left(Y_{e}^{\dagger} A_{e}\right)-12 A_{d} Y_{d}^{\dagger} \operatorname{Tr}\left(A_{d}^{*} Y_{d}^{T}\right)-12 Y_{d} Y_{d}^{\dagger} \operatorname{Tr}\left(A_{d}^{*} A_{d}^{T}\right) \\
& -4 A_{d} Y_{d}^{\dagger} \operatorname{Tr}\left(A_{e}^{*} Y_{e}^{T}\right)-4 Y_{d} Y_{d}^{\dagger} \operatorname{Tr}\left(A_{e}^{*} A_{e}^{T}\right)-12 Y_{d} Y_{d}^{\dagger} \operatorname{Tr}\left(m_{d}^{2} Y_{d} Y_{d}^{\dagger}\right) \\
& -4 Y_{d} Y_{d}^{\dagger} \operatorname{Tr}\left(m_{e}^{2} Y_{e} Y_{e}^{\dagger}\right)-4 Y_{d} Y_{d}^{\dagger} \operatorname{Tr}\left(m_{l}^{2} Y_{e}^{\dagger} Y_{e}\right)-12 Y_{d} Y_{d}^{\dagger} \operatorname{Tr}\left(m_{q}^{2} Y_{d}^{\dagger} Y_{d}\right) \\
& \beta_{m_{u}^{2}}^{(1)}=-\frac{32}{15} g_{1}^{2} \mathbf{1}\left|M_{1}\right|^{2}-\frac{32}{3} g_{3}^{2} \mathbf{1}\left|M_{3}\right|^{2}+4 m_{H_{u}}^{2} Y_{u} Y_{u}^{\dagger}+4 A_{u} A_{u}^{\dagger}+2 m_{u}^{2} Y_{u} Y_{u}^{\dagger}+4 Y_{u} m_{q}^{2} Y_{u}^{\dagger} \\
& +2 Y_{u} Y_{u}^{\dagger} m_{u}^{2}-4 \frac{1}{\sqrt{15}} g_{1} \mathbf{1} \sigma_{1,1} \\
& \beta_{m_{u}^{2}}^{(2)}=+\frac{32}{45} g_{3}^{2}\left(75 g_{3}^{2} M_{3}+8 g_{1}^{2}\left(2 M_{3}+M_{1}\right)\right) \mathbf{1} M_{3}^{*}-\frac{4}{5} g_{1}^{2} m_{H_{u}}^{2} Y_{u} Y_{u}^{\dagger}+12 g_{2}^{2} m_{H_{u}}^{2} Y_{u} Y_{u}^{\dagger} \\
& +24 g_{2}^{2}\left|M_{2}\right|^{2} Y_{u} Y_{u}^{\dagger}-4 m_{H_{d}}^{2}\left|\lambda_{S}\right|^{2} Y_{u} Y_{u}^{\dagger}-8 m_{H_{u}}^{2}\left|\lambda_{S}\right|^{2} Y_{u} Y_{u}^{\dagger} \\
& -4 m_{S}^{2}\left|\lambda_{S}\right|^{2} Y_{u} Y_{u}^{\dagger}-12 m_{H_{d}}^{2}\left|\lambda_{T}\right|^{2} Y_{u} Y_{u}^{\dagger}-24 m_{H_{u}}^{2}\left|\lambda_{T}\right|^{2} Y_{u} Y_{u}^{\dagger} \\
& -12 m_{T}^{2}\left|\lambda_{T}\right|^{2} Y_{u} Y_{u}^{\dagger}-4\left|A_{S}\right|^{2} Y_{u} Y_{u}^{\dagger}-12\left|A_{T}\right|^{2} Y_{u} Y_{u}^{\dagger}+\frac{4}{5} g_{1}^{2} M_{1} Y_{u} A_{u}^{\dagger} \\
& -12 g_{2}^{2} M_{2} Y_{u} A_{u}^{\dagger}-12 g_{2}^{2} M_{2}^{*} A_{u} Y_{u}^{\dagger}-4 \lambda_{S} A_{S}^{*} A_{u} Y_{u}^{\dagger}-12 \lambda_{T} A_{T}^{*} A_{u} Y_{u}^{\dagger}
\end{aligned}
$$




$$
\begin{aligned}
& +\frac{4}{225} g_{1}^{2} M_{1}^{*}\left(45\left(-2 M_{1} Y_{u} Y_{u}^{\dagger}+A_{u} Y_{u}^{\dagger}\right)+8\left(321 g_{1}^{2} M_{1}+40 g_{3}^{2}\left(2 M_{1}+M_{3}\right)\right) \mathbf{1}\right)-\frac{4}{5} g_{1}^{2} A_{u} A_{u}^{\dagger} \\
& +12 g_{2}^{2} A_{u} A_{u}^{\dagger}-4\left|\lambda_{S}\right|^{2} A_{u} A_{u}^{\dagger}-12\left|\lambda_{T}\right|^{2} A_{u} A_{u}^{\dagger}-\frac{2}{5} g_{1}^{2} m_{u}^{2} Y_{u} Y_{u}^{\dagger} \\
& +6 g_{2}^{2} m_{u}^{2} Y_{u} Y_{u}^{\dagger}-2\left|\lambda_{S}\right|^{2} m_{u}^{2} Y_{u} Y_{u}^{\dagger}-6\left|\lambda_{T}\right|^{2} m_{u}^{2} Y_{u} Y_{u}^{\dagger}-\frac{4}{5} g_{1}^{2} Y_{u} m_{q}^{2} Y_{u}^{\dagger} \\
& +12 g_{2}^{2} Y_{u} m_{q}^{2} Y_{u}^{\dagger}-4\left|\lambda_{S}\right|^{2} Y_{u} m_{q}^{2} Y_{u}^{\dagger}-12\left|\lambda_{T}\right|^{2} Y_{u} m_{q}^{2} Y_{u}^{\dagger}-\frac{2}{5} g_{1}^{2} Y_{u} Y_{u}^{\dagger} m_{u}^{2} \\
& +6 g_{2}^{2} Y_{u} Y_{u}^{\dagger} m_{u}^{2}-2\left|\lambda_{S}\right|^{2} Y_{u} Y_{u}^{\dagger} m_{u}^{2}-6\left|\lambda_{T}\right|^{2} Y_{u} Y_{u}^{\dagger} m_{u}^{2} \\
& -4 m_{H_{d}}^{2} Y_{u} Y_{d}^{\dagger} Y_{d} Y_{u}^{\dagger}-4 m_{H_{u}}^{2} Y_{u} Y_{d}^{\dagger} Y_{d} Y_{u}^{\dagger}-4 Y_{u} Y_{d}^{\dagger} A_{d} A_{u}^{\dagger} \\
& -8 m_{H_{u}}^{2} Y_{u} Y_{u}^{\dagger} Y_{u} Y_{u}^{\dagger}-4 Y_{u} Y_{u}^{\dagger} A_{u} A_{u}^{\dagger}-4 Y_{u} A_{d}^{\dagger} A_{d} Y_{u}^{\dagger}-4 Y_{u} A_{u}^{\dagger} A_{u} Y_{u}^{\dagger} \\
& -4 A_{u} Y_{d}^{\dagger} Y_{d} A_{u}^{\dagger}-4 A_{u} Y_{u}^{\dagger} Y_{u} A_{u}^{\dagger}-4 A_{u} A_{d}^{\dagger} Y_{d} Y_{u}^{\dagger}-4 A_{u} A_{u}^{\dagger} Y_{u} Y_{u}^{\dagger} \\
& -2 m_{u}^{2} Y_{u} Y_{d}^{\dagger} Y_{d} Y_{u}^{\dagger}-2 m_{u}^{2} Y_{u} Y_{u}^{\dagger} Y_{u} Y_{u}^{\dagger}-4 Y_{u} m_{q}^{2} Y_{d}^{\dagger} Y_{d} Y_{u}^{\dagger}-4 Y_{u} m_{q}^{2} Y_{u}^{\dagger} Y_{u} Y_{u}^{\dagger} \\
& -4 Y_{u} Y_{d}^{\dagger} m_{d}^{2} Y_{d} Y_{u}^{\dagger}-4 Y_{u} Y_{d}^{\dagger} Y_{d} m_{q}^{2} Y_{u}^{\dagger}-2 Y_{u} Y_{d}^{\dagger} Y_{d} Y_{u}^{\dagger} m_{u}^{2} \\
& -4 Y_{u} Y_{u}^{\dagger} m_{u}^{2} Y_{u} Y_{u}^{\dagger}-4 Y_{u} Y_{u}^{\dagger} Y_{u} m_{q}^{2} Y_{u}^{\dagger}-2 Y_{u} Y_{u}^{\dagger} Y_{u} Y_{u}^{\dagger} m_{u}^{2}-4 \lambda_{S}^{*} Y_{u} A_{u}^{\dagger} A_{S} \\
& -12 \lambda_{T}^{*} Y_{u} A_{u}^{\dagger} A_{T}+\frac{32}{3} g_{3}^{4} \mathbf{1} \sigma_{2,3}+\frac{32}{15} g_{1}^{2} \mathbf{1} \operatorname{Tr} 2 \mathrm{U} 1(1,1)-16 \frac{1}{\sqrt{15}} g_{1} \mathbf{1} \sigma_{3,1}-24 m_{H_{u}}^{2} Y_{u} Y_{u}^{\dagger} \operatorname{Tr}\left(Y_{u} Y_{u}^{\dagger}\right) \\
& -12 A_{u} A_{u}^{\dagger} \operatorname{Tr}\left(Y_{u} Y_{u}^{\dagger}\right)-6 m_{u}^{2} Y_{u} Y_{u}^{\dagger} \operatorname{Tr}\left(Y_{u} Y_{u}^{\dagger}\right)-12 Y_{u} m_{q}^{2} Y_{u}^{\dagger} \operatorname{Tr}\left(Y_{u} Y_{u}^{\dagger}\right) \\
& -6 Y_{u} Y_{u}^{\dagger} m_{u}^{2} \operatorname{Tr}\left(Y_{u} Y_{u}^{\dagger}\right)-12 Y_{u} A_{u}^{\dagger} \operatorname{Tr}\left(Y_{u}^{\dagger} A_{u}\right)-12 A_{u} Y_{u}^{\dagger} \operatorname{Tr}\left(A_{u}^{*} Y_{u}^{T}\right) \\
& -12 Y_{u} Y_{u}^{\dagger} \operatorname{Tr}\left(A_{u}^{*} A_{u}^{T}\right)-12 Y_{u} Y_{u}^{\dagger} \operatorname{Tr}\left(m_{q}^{2} Y_{u}^{\dagger} Y_{u}\right)-12 Y_{u} Y_{u}^{\dagger} \operatorname{Tr}\left(m_{u}^{2} Y_{u} Y_{u}^{\dagger}\right) \\
& \beta_{m_{e}^{2}}^{(1)}=-\frac{24}{5} g_{1}^{2} \mathbf{1}\left|M_{1}\right|^{2}+2\left(2 A_{e} A_{e}^{\dagger}+2 m_{H_{d}}^{2} Y_{e} Y_{e}^{\dagger}+2 Y_{e} m_{l}^{2} Y_{e}^{\dagger}+m_{e}^{2} Y_{e} Y_{e}^{\dagger}+Y_{e} Y_{e}^{\dagger} m_{e}^{2}\right) \\
& +2 \sqrt{\frac{3}{5}} g_{1} \mathbf{1} \sigma_{1,1} \\
& \beta_{m_{e}^{2}}^{(2)}=\frac{2}{25}\left(6 g_{1}^{2} M_{1}^{*}\left(234 g_{1}^{2} M_{1} \mathbf{1}+5\left(-2 M_{1} Y_{e} Y_{e}^{\dagger}+A_{e} Y_{e}^{\dagger}\right)\right)+20 g_{1} \mathbf{1}\left(3 g_{1} \operatorname{Tr} 2 \mathrm{U} 1(1,1)+\sqrt{15} \sigma_{3,1}\right)\right. \\
& -5\left(30 g_{2}^{2} M_{2}^{*} A_{e} Y_{e}^{\dagger}+10 \lambda_{S} A_{S}^{*} A_{e} Y_{e}^{\dagger}+30 \lambda_{T} A_{T}^{*} A_{e} Y_{e}^{\dagger}+6 g_{1}^{2} A_{e} A_{e}^{\dagger}\right. \\
& -30 g_{2}^{2} A_{e} A_{e}^{\dagger}+10\left|\lambda_{S}\right|^{2} A_{e} A_{e}^{\dagger}+30\left|\lambda_{T}\right|^{2} A_{e} A_{e}^{\dagger}+3 g_{1}^{2} m_{e}^{2} Y_{e} Y_{e}^{\dagger} \\
& -15 g_{2}^{2} m_{e}^{2} Y_{e} Y_{e}^{\dagger}+5\left|\lambda_{S}\right|^{2} m_{e}^{2} Y_{e} Y_{e}^{\dagger}+15\left|\lambda_{T}\right|^{2} m_{e}^{2} Y_{e} Y_{e}^{\dagger}+6 g_{1}^{2} Y_{e} m_{l}^{2} Y_{e}^{\dagger} \\
& -30 g_{2}^{2} Y_{e} m_{l}^{2} Y_{e}^{\dagger}+10\left|\lambda_{S}\right|^{2} Y_{e} m_{l}^{2} Y_{e}^{\dagger}+30\left|\lambda_{T}\right|^{2} Y_{e} m_{l}^{2} Y_{e}^{\dagger}+3 g_{1}^{2} Y_{e} Y_{e}^{\dagger} m_{e}^{2} \\
& -15 g_{2}^{2} Y_{e} Y_{e}^{\dagger} m_{e}^{2}+5\left|\lambda_{S}\right|^{2} Y_{e} Y_{e}^{\dagger} m_{e}^{2}+15\left|\lambda_{T}\right|^{2} Y_{e} Y_{e}^{\dagger} m_{e}^{2}+20 m_{H_{d}}^{2} Y_{e} Y_{e}^{\dagger} Y_{e} Y_{e}^{\dagger} \\
& +10 Y_{e} Y_{e}^{\dagger} A_{e} A_{e}^{\dagger}+10 Y_{e} A_{e}^{\dagger} A_{e} Y_{e}^{\dagger}+10 A_{e} Y_{e}^{\dagger} Y_{e} A_{e}^{\dagger}+10 A_{e} A_{e}^{\dagger} Y_{e} Y_{e}^{\dagger} \\
& +5 m_{e}^{2} Y_{e} Y_{e}^{\dagger} Y_{e} Y_{e}^{\dagger}+10 Y_{e} m_{l}^{2} Y_{e}^{\dagger} Y_{e} Y_{e}^{\dagger}+10 Y_{e} Y_{e}^{\dagger} m_{e}^{2} Y_{e} Y_{e}^{\dagger}+10 Y_{e} Y_{e}^{\dagger} Y_{e} m_{l}^{2} Y_{e}^{\dagger} \\
& +5 Y_{e} Y_{e}^{\dagger} Y_{e} Y_{e}^{\dagger} m_{e}^{2}+30 A_{e} A_{e}^{\dagger} \operatorname{Tr}\left(Y_{d} Y_{d}^{\dagger}\right)+15 m_{e}^{2} Y_{e} Y_{e}^{\dagger} \operatorname{Tr}\left(Y_{d} Y_{d}^{\dagger}\right) \\
& +30 Y_{e} m_{l}^{2} Y_{e}^{\dagger} \operatorname{Tr}\left(Y_{d} Y_{d}^{\dagger}\right)+15 Y_{e} Y_{e}^{\dagger} m_{e}^{2} \operatorname{Tr}\left(Y_{d} Y_{d}^{\dagger}\right)+10 A_{e} A_{e}^{\dagger} \operatorname{Tr}\left(Y_{e} Y_{e}^{\dagger}\right) \\
& +5 m_{e}^{2} Y_{e} Y_{e}^{\dagger} \operatorname{Tr}\left(Y_{e} Y_{e}^{\dagger}\right)+10 Y_{e} m_{l}^{2} Y_{e}^{\dagger} \operatorname{Tr}\left(Y_{e} Y_{e}^{\dagger}\right)+5 Y_{e} Y_{e}^{\dagger} m_{e}^{2} \operatorname{Tr}\left(Y_{e} Y_{e}^{\dagger}\right) \\
& +Y_{e} A_{e}^{\dagger}\left(10 \lambda_{S}^{*} A_{S}+10 \operatorname{Tr}\left(Y_{e}^{\dagger} A_{e}\right)+30 g_{2}^{2} M_{2}+30 \lambda_{T}^{*} A_{T}+30 \operatorname{Tr}\left(Y_{d}^{\dagger} A_{d}\right)-6 g_{1}^{2} M_{1}\right) \\
& +30 A_{e} Y_{e}^{\dagger} \operatorname{Tr}\left(A_{d}^{*} Y_{d}^{T}\right)+10 A_{e} Y_{e}^{\dagger} \operatorname{Tr}\left(A_{e}^{*} Y_{e}^{T}\right) \\
& +2 Y_{e} Y_{e}^{\dagger}\left(3 g_{1}^{2} m_{H_{d}}^{2}-15 g_{2}^{2} m_{H_{d}}^{2}-30 g_{2}^{2}\left|M_{2}\right|^{2}+5\left(2 m_{H_{d}}^{2}+m_{H_{u}}^{2}+m_{S}^{2}\right)\left|\lambda_{S}\right|^{2}\right.
\end{aligned}
$$




$$
\begin{aligned}
& +30 m_{H_{d}}^{2}\left|\lambda_{T}\right|^{2}+15 m_{H_{u}}^{2}\left|\lambda_{T}\right|^{2} \\
& +15 m_{T}^{2}\left|\lambda_{T}\right|^{2}+5\left|A_{S}\right|^{2}+15\left|A_{T}\right|^{2}+30 m_{H_{d}}^{2} \operatorname{Tr}\left(Y_{d} Y_{d}^{\dagger}\right)+10 m_{H_{d}}^{2} \operatorname{Tr}\left(Y_{e} Y_{e}^{\dagger}\right)+15 \operatorname{Tr}\left(A_{d}^{*} A_{d}^{T}\right) \\
& \left.\left.\left.+5 \operatorname{Tr}\left(A_{e}^{*} A_{e}^{T}\right)+15 \operatorname{Tr}\left(m_{d}^{2} Y_{d} Y_{d}^{\dagger}\right)+5 \operatorname{Tr}\left(m_{e}^{2} Y_{e} Y_{e}^{\dagger}\right)+5 \operatorname{Tr}\left(m_{l}^{2} Y_{e}^{\dagger} Y_{e}\right)+15 \operatorname{Tr}\left(m_{q}^{2} Y_{d}^{\dagger} Y_{d}\right)\right)\right)\right) \\
& \beta_{m_{S}^{2}}^{(1)}=4\left(3 m_{S}^{2}\left|\kappa_{S}\right|^{2}+\left(m_{H_{d}}^{2}+m_{H_{u}}^{2}+m_{S}^{2}\right)\left|\lambda_{S}\right|^{2}+\left|A_{\kappa}\right|^{2}+\left|A_{S}\right|^{2}\right) \\
& \beta_{m_{S}^{2}}^{(2)}=-\frac{4}{5}\left(120 m_{S}^{2} \kappa_{S}^{2} \kappa_{S}^{*, 2}+20\left(m_{H_{d}}^{2}+m_{H_{u}}^{2}+m_{S}^{2}\right) \lambda_{S}^{2} \lambda_{S}^{*, 2}\right. \\
& +20 \kappa_{S}^{*}\left(4 \kappa_{S}\left|A_{\kappa}\right|^{2}+\left(4 m_{S}^{2}+m_{H_{d}}^{2}+m_{H_{u}}^{2}\right) \kappa_{S}\left|\lambda_{S}\right|^{2}+A_{S}^{*}\left(\kappa_{S} A_{S}+\lambda_{S} A_{\kappa}\right)\right) \\
& +A_{S}^{*}\left(A_{S}\left(-15 g_{2}^{2}+15 \operatorname{Tr}\left(Y_{d} Y_{d}^{\dagger}\right)+15 \operatorname{Tr}\left(Y_{u} Y_{u}^{\dagger}\right)+30\left|\lambda_{T}\right|^{2}-3 g_{1}^{2}+5 \operatorname{Tr}\left(Y_{e} Y_{e}^{\dagger}\right)\right)\right. \\
& \left.+\lambda_{S}\left(15 g_{2}^{2} M_{2}+15 \operatorname{Tr}\left(Y_{d}^{\dagger} A_{d}\right)+15 \operatorname{Tr}\left(Y_{u}^{\dagger} A_{u}\right)+30 \lambda_{T}^{*} A_{T}+3 g_{1}^{2} M_{1}+5 \operatorname{Tr}\left(Y_{e}^{\dagger} A_{e}\right)\right)\right) \\
& +\lambda_{S}^{*}\left(-3 g_{1}^{2} m_{H_{d}}^{2} \lambda_{S}-15 g_{2}^{2} m_{H_{d}}^{2} \lambda_{S}-3 g_{1}^{2} m_{H_{u}}^{2} \lambda_{S}-15 g_{2}^{2} m_{H_{u}}^{2} \lambda_{S}-3 g_{1}^{2} m_{S}^{2} \lambda_{S}-15 g_{2}^{2} m_{S}^{2} \lambda_{S}\right. \\
& +60 m_{H_{d}}^{2} \lambda_{S}\left|\lambda_{T}\right|^{2}+60 m_{H_{u}}^{2} \lambda_{S}\left|\lambda_{T}\right|^{2}+30 m_{S}^{2} \lambda_{S}\left|\lambda_{T}\right|^{2}+30 m_{T}^{2} \lambda_{S}\left|\lambda_{T}\right|^{2}+20 \lambda_{S}\left|A_{\kappa}\right|^{2}+40 \lambda_{S}\left|A_{S}\right|^{2} \\
& +30 \lambda_{S}\left|A_{T}\right|^{2}+20 \kappa_{S} A_{\kappa}^{*} A_{S}+30 \lambda_{T} A_{T}^{*} A_{S}+3 g_{1}^{2} M_{1}^{*}\left(-2 M_{1} \lambda_{S}+A_{S}\right) \\
& +15 g_{2}^{2} M_{2}^{*}\left(-2 M_{2} \lambda_{S}+A_{S}\right) \\
& +30 m_{H_{d}}^{2} \lambda_{S} \operatorname{Tr}\left(Y_{d} Y_{d}^{\dagger}\right)+15 m_{H_{u}}^{2} \lambda_{S} \operatorname{Tr}\left(Y_{d} Y_{d}^{\dagger}\right)+15 m_{S}^{2} \lambda_{S} \operatorname{Tr}\left(Y_{d} Y_{d}^{\dagger}\right)+10 m_{H_{d}}^{2} \lambda_{S} \operatorname{Tr}\left(Y_{e} Y_{e}^{\dagger}\right) \\
& +5 m_{H_{u}}^{2} \lambda_{S} \operatorname{Tr}\left(Y_{e} Y_{e}^{\dagger}\right)+5 m_{S}^{2} \lambda_{S} \operatorname{Tr}\left(Y_{e} Y_{e}^{\dagger}\right)+15 m_{H_{d}}^{2} \lambda_{S} \operatorname{Tr}\left(Y_{u} Y_{u}^{\dagger}\right)+30 m_{H_{u}}^{2} \lambda_{S} \operatorname{Tr}\left(Y_{u} Y_{u}^{\dagger}\right) \\
& +15 m_{S}^{2} \lambda_{S} \operatorname{Tr}\left(Y_{u} Y_{u}^{\dagger}\right)+15 A_{S} \operatorname{Tr}\left(A_{d}^{*} Y_{d}^{T}\right)+15 \lambda_{S} \operatorname{Tr}\left(A_{d}^{*} A_{d}^{T}\right)+5 A_{S} \operatorname{Tr}\left(A_{e}^{*} Y_{e}^{T}\right)+5 \lambda_{S} \operatorname{Tr}\left(A_{e}^{*} A_{e}^{T}\right) \\
& +15 A_{S} \operatorname{Tr}\left(A_{u}^{*} Y_{u}^{T}\right)+15 \lambda_{S} \operatorname{Tr}\left(A_{u}^{*} A_{u}^{T}\right)+15 \lambda_{S} \operatorname{Tr}\left(m_{d}^{2} Y_{d} Y_{d}^{\dagger}\right) \\
& +5 \lambda_{S} \operatorname{Tr}\left(m_{e}^{2} Y_{e} Y_{e}^{\dagger}\right) \\
& \left.\left.+5 \lambda_{S} \operatorname{Tr}\left(m_{l}^{2} Y_{e}^{\dagger} Y_{e}\right)+15 \lambda_{S} \operatorname{Tr}\left(m_{q}^{2} Y_{d}^{\dagger} Y_{d}\right)+15 \lambda_{S} \operatorname{Tr}\left(m_{q}^{2} Y_{u}^{\dagger} Y_{u}\right)+15 \lambda_{S} \operatorname{Tr}\left(m_{u}^{2} Y_{u} Y_{u}^{\dagger}\right)\right)\right) \\
& \beta_{m_{T}^{2}}^{(1)}=4\left(-4 g_{2}^{2}\left|M_{2}\right|^{2}+\left(m_{H_{d}}^{2}+m_{H_{u}}^{2}+m_{T}^{2}\right)\left|\lambda_{T}\right|^{2}+\left|A_{T}\right|^{2}\right) \\
& \beta_{m_{T}^{2}}^{(2)}=-\frac{4}{5}\left(-3 g_{1}^{2}\left|A_{T}\right|^{2}+5 g_{2}^{2}\left|A_{T}\right|^{2}+60\left(m_{H_{d}}^{2}+m_{H_{u}}^{2}+m_{T}^{2}\right) \lambda_{T}^{2} \lambda_{T}^{*, 2}+3 g_{1}^{2} M_{1} \lambda_{T} A_{T}^{*}-5 g_{2}^{2} M_{2} \lambda_{T} A_{T}^{*}\right. \\
& +10 \lambda_{T} \lambda_{S}^{*} A_{T}^{*} A_{S}+10\left|\lambda_{S}\right|^{2} A_{T}^{*} A_{T}-5 g_{2}^{2} M_{2}^{*}\left(76 g_{2}^{2} M_{2}+\lambda_{T}^{*}\left(-2 M_{2} \lambda_{T}+A_{T}\right)\right)-20 g_{2}^{4} \sigma_{2,2} \\
& +15\left|A_{T}\right|^{2} \operatorname{Tr}\left(Y_{d} Y_{d}^{\dagger}\right)+5\left|A_{T}\right|^{2} \operatorname{Tr}\left(Y_{e} Y_{e}^{\dagger}\right)+15\left|A_{T}\right|^{2} \operatorname{Tr}\left(Y_{u} Y_{u}^{\dagger}\right)+15 \lambda_{T} A_{T}^{*} \operatorname{Tr}\left(Y_{d}^{\dagger} A_{d}\right) \\
& +5 \lambda_{T} A_{T}^{*} \operatorname{Tr}\left(Y_{e}^{\dagger} A_{e}\right)+15 \lambda_{T} A_{T}^{*} \operatorname{Tr}\left(Y_{u}^{\dagger} A_{u}\right) \\
& +\lambda_{T}^{*}\left(-3 g_{1}^{2} m_{H_{d}}^{2} \lambda_{T}+5 g_{2}^{2} m_{H_{d}}^{2} \lambda_{T}-3 g_{1}^{2} m_{H_{u}}^{2} \lambda_{T}+5 g_{2}^{2} m_{H_{u}}^{2} \lambda_{T}-3 g_{1}^{2} m_{T}^{2} \lambda_{T}+5 g_{2}^{2} m_{T}^{2} \lambda_{T}\right. \\
& +10\left(2 m_{H_{d}}^{2}+2 m_{H_{u}}^{2}+m_{S}^{2}+m_{T}^{2}\right) \lambda_{T}\left|\lambda_{S}\right|^{2}+10 \lambda_{T}\left|A_{S}\right|^{2}+120 \lambda_{T}\left|A_{T}\right|^{2} \\
& +10 \lambda_{S} A_{S}^{*} A_{T}+3 g_{1}^{2} M_{1}^{*}\left(-2 M_{1} \lambda_{T}+A_{T}\right) \\
& +30 m_{H_{d}}^{2} \lambda_{T} \operatorname{Tr}\left(Y_{d} Y_{d}^{\dagger}\right)+15 m_{H_{u}}^{2} \lambda_{T} \operatorname{Tr}\left(Y_{d} Y_{d}^{\dagger}\right)+15 m_{T}^{2} \lambda_{T} \operatorname{Tr}\left(Y_{d} Y_{d}^{\dagger}\right)+10 m_{H_{d}}^{2} \lambda_{T} \operatorname{Tr}\left(Y_{e} Y_{e}^{\dagger}\right) \\
& +5 m_{H_{u}}^{2} \lambda_{T} \operatorname{Tr}\left(Y_{e} Y_{e}^{\dagger}\right)+5 m_{T}^{2} \lambda_{T} \operatorname{Tr}\left(Y_{e} Y_{e}^{\dagger}\right)+15 m_{H_{d}}^{2} \lambda_{T} \operatorname{Tr}\left(Y_{u} Y_{u}^{\dagger}\right)+30 m_{H_{u}}^{2} \lambda_{T} \operatorname{Tr}\left(Y_{u} Y_{u}^{\dagger}\right) \\
& +15 m_{T}^{2} \lambda_{T} \operatorname{Tr}\left(Y_{u} Y_{u}^{\dagger}\right)+15 A_{T} \operatorname{Tr}\left(A_{d}^{*} Y_{d}^{T}\right)+15 \lambda_{T} \operatorname{Tr}\left(A_{d}^{*} A_{d}^{T}\right)+5 A_{T} \operatorname{Tr}\left(A_{e}^{*} Y_{e}^{T}\right)
\end{aligned}
$$




$$
\begin{aligned}
& +5 \lambda_{T} \operatorname{Tr}\left(A_{e}^{*} A_{e}^{T}\right) \\
& +15 A_{T} \operatorname{Tr}\left(A_{u}^{*} Y_{u}^{T}\right)+15 \lambda_{T} \operatorname{Tr}\left(A_{u}^{*} A_{u}^{T}\right)+15 \lambda_{T} \operatorname{Tr}\left(m_{d}^{2} Y_{d} Y_{d}^{\dagger}\right)+5 \lambda_{T} \operatorname{Tr}\left(m_{e}^{2} Y_{e} Y_{e}^{\dagger}\right) \\
& \left.\left.+5 \lambda_{T} \operatorname{Tr}\left(m_{l}^{2} Y_{e}^{\dagger} Y_{e}\right)+15 \lambda_{T} \operatorname{Tr}\left(m_{q}^{2} Y_{d}^{\dagger} Y_{d}\right)+15 \lambda_{T} \operatorname{Tr}\left(m_{q}^{2} Y_{u}^{\dagger} Y_{u}\right)+15 \lambda_{T} \operatorname{Tr}\left(m_{u}^{2} Y_{u} Y_{u}^{\dagger}\right)\right)\right) \\
\beta_{m_{O}^{2}}^{(1)}= & -24 g_{3}^{2}\left|M_{3}\right|^{2} \\
\beta_{m_{O}^{2}}^{(2)}= & 24 g_{3}^{4}\left(15\left|M_{3}\right|^{2}+\sigma_{2,3}\right)
\end{aligned}
$$

Open Access. This article is distributed under the terms of the Creative Commons Attribution License which permits any use, distribution and reproduction in any medium, provided the original author(s) and source are credited.

\section{References}

[1] P. Fayet, Massive gluinos, Phys. Lett. B 78 (1978) 417 [inSPIRE].

[2] J. Polchinski and L. Susskind, Breaking of supersymmetry at intermediate-energy, Phys. Rev. D 26 (1982) 3661 [inSPIRE].

[3] L. Hall and L. Randall, U(1) $)_{R}$ symmetric supersymmetry, Nucl. Phys. B 352 (1991) 289 [INSPIRE].

[4] P.J. Fox, A.E. Nelson and N. Weiner, Dirac gaugino masses and supersoft supersymmetry breaking, JHEP 08 (2002) 035 [hep-ph/0206096] [INSPIRE].

[5] A.E. Nelson, N. Rius, V. Sanz and M. Ünsal, The minimal supersymmetric model without a $\mu$ term, JHEP 08 (2002) 039 [hep-ph/0206102] [INSPIRE].

[6] I. Antoniadis, A. Delgado, K. Benakli, M. Quirós and M. Tuckmantel, Splitting extended supersymmetry, Phys. Lett. B 634 (2006) 302 [hep-ph/0507192] [INSPIRE].

[7] I. Antoniadis, K. Benakli, A. Delgado, M. Quirós and M. Tuckmantel, Split extended supersymmetry from intersecting branes, Nucl. Phys. B 744 (2006) 156 [hep-th/0601003] [INSPIRE].

[8] I. Antoniadis, K. Benakli, A. Delgado and M. Quirós, A new gauge mediation theory, Adv. Stud. Theor. Phys. 2 (2008) 645 [hep-ph/0610265] [INSPIRE].

[9] K. Hsieh, Pseudo-Dirac bino dark matter, Phys. Rev. D 77 (2008) 015004 [arXiv:0708.3970] [INSPIRE].

[10] S.D.L. Amigo, A.E. Blechman, P.J. Fox and E. Poppitz, R-symmetric gauge mediation, JHEP 01 (2009) 018 [arXiv:0809.1112] [INSPIRE].

[11] A.E. Blechman, R-symmetric gauge mediation and the minimal $R$-symmetric supersymmetric standard model, Mod. Phys. Lett. A 24 (2009) 633 [arXiv:0903.2822] [inSPIRE].

[12] K. Benakli and M. Goodsell, Dirac gauginos in general gauge mediation, Nucl. Phys. B 816 (2009) 185 [arXiv:0811.4409] [INSPIRE].

[13] G. Bélanger, K. Benakli, M. Goodsell, C. Moura and A. Pukhov, Dark matter with Dirac and Majorana gaugino masses, JCAP 08 (2009) 027 [arXiv:0905.1043] [INSPIRE].

[14] K. Benakli and M. Goodsell, Dirac gauginos and kinetic mixing, Nucl. Phys. B 830 (2010) 315 [arXiv:0909.0017] [INSPIRE]. 
[15] E.J. Chun, J.-C. Park and S. Scopel, Dirac gaugino as leptophilic dark matter, JCAP 02 (2010) 015 [arXiv:0911.5273] [INSPIRE].

[16] K. Benakli and M. Goodsell, Dirac gauginos, gauge mediation and unification, Nucl. Phys. B 840 (2010) 1 [arXiv:1003.4957] [InSPIRE].

[17] L.M. Carpenter, Dirac gauginos, negative supertraces and gauge mediation, JHEP 09 (2012) 102 [arXiv: 1007.0017] [INSPIRE].

[18] G.D. Kribs, T. Okui and T.S. Roy, Viable gravity-mediated supersymmetry breaking, Phys. Rev. D 82 (2010) 115010 [arXiv: 1008.1798] [INSPIRE].

[19] E.J. Chun, Leptogenesis origin of Dirac gaugino dark matter, Phys. Rev. D 83 (2011) 053004 [arXiv: 1009.0983] [InSPIRE].

[20] S. Choi, D. Choudhury, A. Freitas, J. Kalinowski and P. Zerwas, The extended Higgs system in R-symmetric supersymmetry theories, Phys. Lett. B 697 (2011) 215 [Erratum ibid. B 698 (2011) 457] [arXiv : 1012.2688] [INSPIRE].

[21] K. Benakli, M.D. Goodsell and A.-K. Maier, Generating $\mu$ and B $\mu$ in models with Dirac Gauginos, Nucl. Phys. B 851 (2011) 445 [arXiv:1104.2695] [INSPIRE].

[22] S. Abel and M. Goodsell, Easy Dirac gauginos, JHEP 06 (2011) 064 [arXiv:1102.0014] [INSPIRE].

[23] P. Kumar and E. Ponton, Electroweak baryogenesis and dark matter with an approximate R-symmetry, JHEP 11 (2011) 037 [arXiv:1107.1719] [INSPIRE].

[24] K. Benakli, Dirac gauginos: a user manual, Fortsch. Phys. 59 (2011) 1079 [arXiv: 1106.1649] [INSPIRE].

[25] R. Davies, J. March-Russell and M. McCullough, A supersymmetric one Higgs doublet model, JHEP 04 (2011) 108 [arXiv: 1103.1647] [INSPIRE].

[26] R. Davies and M. McCullough, Small neutrino masses due to R-symmetry breaking for a small cosmological constant, Phys. Rev. D 86 (2012) 025014 [arXiv:1111.2361] [InSPIRE].

[27] M. Heikinheimo, M. Kellerstein and V. Sanz, How many supersymmetries?, JHEP 04 (2012) 043 [arXiv:1111.4322] [INSPIRE].

[28] K. Rehermann and C.M. Wells, Weak Scale Leptogenesis, R-symmetry and a Displaced Higgs, arXiv: 1111.0008 [INSPIRE].

[29] G.D. Kribs and A. Martin, Supersoft supersymmetry is super-safe, Phys. Rev. D 85 (2012) 115014 [arXiv:1203.4821] [InSPIRE].

[30] R. Davies, Dirac gauginos and unification in F-theory, JHEP 10 (2012) 010 [arXiv: 1205.1942] [INSPIRE].

[31] R. Argurio, M. Bertolini, L. Di Pietro, F. Porri and D. Redigolo, Holographic correlators for general gauge mediation, JHEP 08 (2012) 086 [arXiv: 1205.4709] [INSPIRE].

[32] S. Choi, J. Kalinowski, J. Kim and E. Popenda, Scalar gluons and Dirac gluinos at the LHC, Acta Phys. Polon. B 40 (2009) 2913 [arXiv:0911.1951] [InSPIRE].

[33] S. Choi et al., Dirac neutralinos and electroweak scalar bosons of $N=1 / N=2$ hybrid supersymmetry at colliders, JHEP 08 (2010) 025 [arXiv: 1005.0818] [INSPIRE].

[34] J. Kalinowski, Phenomenology of R-symmetric supersymmetry, Acta Phys. Polon. B 42 (2011) 2425 [InSPIRE]. 
[35] D. Goncalves-Netto, D. Lopez-Val, K. Mawatari, T. Plehn and I. Wigmore, Sgluon pair production to next-to-leading order, Phys. Rev. D 85 (2012) 114024 [arXiv:1203.6358] [INSPIRE].

[36] M. Papucci, J.T. Ruderman and A. Weiler, Natural SUSY endures, JHEP 09 (2012) 035 [arXiv:1110.6926] [INSPIRE].

[37] I. Jack and D. Jones, Soft supersymmetry breaking and finiteness, Phys. Lett. B 333 (1994) 372 [hep-ph/9405233] [INSPIRE].

[38] S.P. Martin and M.T. Vaughn, Two loop renormalization group equations for soft supersymmetry breaking couplings, Phys. Rev. D 50 (1994) 2282 [Erratum ibid. D 78 (2008) 039903] [hep-ph/9311340] [INSPIRE].

[39] Y. Yamada, Two loop renormalization group equations for soft SUSY breaking scalar interactions: supergraph method, Phys. Rev. D 50 (1994) 3537 [hep-ph/9401241] [INSPIRE].

[40] I. Jack, D. Jones and R. Wild, Gauge singlet renormalization in softly broken supersymmetric theories, Phys. Lett. B 509 (2001) 131 [hep-ph/0103255] [INSPIRE].

[41] I. Jack and D. Jones, Nonstandard soft supersymmetry breaking, Phys. Lett. B 457 (1999) 101 [hep-ph/9903365] [INSPIRE].

[42] I. Jack and D. Jones, Quasiinfrared fixed points and renormalization group invariant trajectories for nonholomorphic soft supersymmetry breaking,

Phys. Rev. D 61 (2000) 095002 [hep-ph/9909570] [INSPIRE].

[43] M.E. Machacek and M.T. Vaughn, Two Loop Renormalization Group Equations in a General Quantum Field Theory. 1. Wave Function Renormalization, Nucl. Phys. B 222 (1983) 83 [INSPIRE].

[44] M.E. Machacek and M.T. Vaughn, Two Loop Renormalization Group Equations in a General Quantum Field Theory. 2. Yukawa Couplings, Nucl. Phys. B 236 (1984) 221 [INSPIRE].

[45] M.E. Machacek and M.T. Vaughn, Two Loop Renormalization Group Equations in a General Quantum Field Theory. 3. Scalar Quartic Couplings, Nucl. Phys. B 249 (1985) 70 [inSPIRE].

[46] M.-x. Luo, H.-w. Wang and Y. Xiao, Two loop renormalization group equations in general gauge field theories, Phys. Rev. D 67 (2003) 065019 [hep-ph/0211440] [InSPIRE].

[47] S.P. Martin and M.T. Vaughn, Regularization dependence of running couplings in softly broken supersymmetry, Phys. Lett. B 318 (1993) 331 [hep-ph/9308222] [INSPIRE].

[48] I. Jack and D. Jones, The Fayet-Iliopoulos D term and its renormalization in the MSSM, Phys. Rev. D 63 (2001) 075010 [hep-ph/0010301] [inSPIRE].

[49] R.M. Fonseca, M. Malinsky, W. Porod and F. Staub, Running soft parameters in SUSY models with multiple U(1) gauge factors, Nucl. Phys. B 854 (2012) 28 [arXiv:1107.2670] [INSPIRE].

[50] F. Staub, Sarah, arXiv:0806.0538 [INSPIRE].

[51] F. Staub, Automatic calculation of supersymmetric renormalization group equations and self energies, Comput. Phys. Commun. 182 (2011) 808 [arXiv:1002.0840] [INSPIRE].

[52] K. Benakli, M.D. Goodsell and F. Staub, Dirac gauginos and the $125 \mathrm{GeV}$ Higgs, arXiv: 1211.0552 [INSPIRE].

[53] K. Benakli, M.D. Goodsell, J.S. Kim, W. Porod and F. Staub, work in progress. 